\title{
Type II Supernova Spectral Diversity. I. Observations, Sample Characterization, and Spectral Line Evolution*
}

\author{
Claudia P. Gutiérrez ${ }^{1,2,3,4}$ (1) , Joseph P. Anderson ${ }^{3}$ (10), Mario Hamuy ${ }^{1,2}$, Nidia Morrel1 ${ }^{5}$ (10), Santiago González-Gaitan ${ }^{1,6}$,

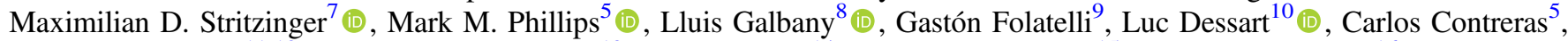

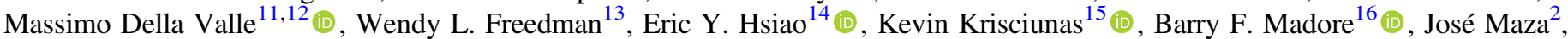 \\ Nicholas B. Suntzeff ${ }^{15}$, Jose Luis Prieto ${ }^{1,17}$ (10) Luis González $^{2}$, Enrico Cappellaro ${ }^{18}$ (1) , Mauricio Navarrete $^{5}$, \\ Alessandro Pizzella ${ }^{19}$ (1), Maria T. Ruiz ${ }^{2}$, R. Chris Smith ${ }^{20}$, and Massimo Turatto ${ }^{18}$ \\ ${ }^{1}$ Millennium Institute of Astrophysics, Casilla 36-D, Santiago, Chile \\ 2 Departamento de Astronomía, Universidad de Chile, Casilla 36-D, Santiago, Chile \\ ${ }^{3}$ European Southern Observatory, Alonso de Córdova 3107, Casilla 19, Santiago, Chile \\ ${ }^{4}$ Department of Physics and Astronomy, University of Southampton, Southampton, SO17 1BJ, UK; C.P.Gutierrez-Avendano@soton.ac.uk \\ 5 Carnegie Observatories, Las Campanas Observatory, Casilla 601, La Serena, Chile \\ ${ }^{6}$ Center for Mathematical Modelling, University of Chile, Beauchef 851, Santiago, Chile \\ ${ }^{7}$ Department of Physics and Astronomy, Aarhus University, Ny Munkegade 120, DK-8000 Aarhus C, Denmark \\ 8 PITT PACC, Department of Physics and Astronomy, University of Pittsburgh, Pittsburgh, PA 15260, USA \\ ${ }^{9}$ Facultad de Ciencias Astronómicas y Geofísicas, Universidad Nacional de La Plata, Instituto de Astrofísica de La Plata (IALP), \\ CONICET, Paseo del Bosque SN, B1900FWA La Plata, Argentina
${ }^{10}$ Unidad Mixta Internacional Franco-Chilena de Astronomía (CNRS UMI 3386), Departamento de Astronomía, Universidad de Chile, \\ Camino El Observatorio 1515, Las Condes, Santiago, Chile \\ ${ }^{11}$ INAF, Osservatorio Astronomico di Capodimonte, salita Moiariello 16, I-80131 Napoli, Italy \\ ${ }^{12}$ International Center for Relativistic Astrophysics, Piazzale della Repubblica 2, I-65122 Pescara, Italy \\ ${ }^{13}$ Department of Astronomy and Astrophysics, University of Chicago, 5640 South Ellis Avenue, Chicago, IL 60637, USA \\ ${ }^{14}$ Department of Physics, Florida State University, Tallahassee, FL 32306, USA \\ 15 Department of Physics and Astronomy, Texas A\&M University, College Station, TX 77843, USA \\ ${ }^{16}$ Observatories of the Carnegie Institution for Science, Pasadena, CA 91101, USA
${ }^{17}$ Núcleo de Astronomía de la Facultad de Ingeniería y Ciencias, Universidad Diego Portales, Av. Ejército 441, Santiago, Chile \\ ${ }^{18}$ INAF, Osservatorio Astronomico di Padova, Vicolo dell'Osservatorio 5, 35122 Padova, Italy \\ ${ }^{19}$ Dipartimento di Fisica e Astronomia-Universita di Padova, Vicolo dellOsservatorio 3, I-35122 Padova, Italy \\ ${ }^{20}$ Cerro Tololo Inter-American Observatory, National Optical Astronomy Observatory, Casilla 603, La Serena, Chile \\ Received 2017 June 29; revised 2017 August 21; accepted 2017 September 1; published 2017 November 21
}

\begin{abstract}
We present 888 visual-wavelength spectra of 122 nearby type II supernovae (SNe II) obtained between 1986 and 2009, and ranging between 3 and 363 days post-explosion. In this first paper, we outline our observations and data reduction techniques, together with a characterization based on the spectral diversity of SNe II. A statistical analysis of the spectral matching technique is discussed as an alternative to nondetection constraints for estimating $\mathrm{SN}$ explosion epochs. The time evolution of spectral lines is presented and analyzed in terms of how this differs for $\mathrm{SNe}$ of different photometric, spectral, and environmental properties: velocities, pseudo-equivalent widths, decline rates, magnitudes, time durations, and environment metallicity. Our sample displays a large range in ejecta expansion velocities, from $\sim 9600$ to $\sim 1500 \mathrm{~km} \mathrm{~s}^{-1}$ at 50 days post-explosion with a median $\mathrm{H}_{\alpha}$ value of $7300 \mathrm{~km} \mathrm{~s}^{-1}$. This is most likely explained through differing explosion energies. Significant diversity is also observed in the absolute strength of spectral lines, characterized through their pseudo-equivalent widths. This implies significant diversity in both temperature evolution (linked to progenitor radius) and progenitor metallicity between different SNe II. Around 60\% of our sample shows an extra absorption component on the blue side of the $\mathrm{H}_{\alpha}$ P-Cygni profile ("Cachito" feature) between 7 and 120 days since explosion. Studying the nature of Cachito, we conclude that these features at early times (before $\sim 35$ days) are associated with Si II $\lambda 6355$, while past the middle of the plateau phase they are related to high velocity (HV) features of hydrogen lines.
\end{abstract}

Key words: supernovae: general - surveys - techniques: photometric - techniques: spectroscopic

Supporting material: figure set, machine-readable tables

\section{Introduction}

Supernovae (SNe) exhibiting prevalent Balmer lines in their spectra are known as Type II SNe (SNe II henceforth, Minkowski 1941). They are produced by the explosion of

* This paper includes data gathered with the $6.5 \mathrm{~m}$ Magellan Telescopes located at Las Campanas Observatory, Chile; and the Gemini Observatory, Cerro Pachon, Chile (Gemini Program GS-2008B-Q-56). Based on observations collected at the European Organisation for Astronomical Research in the Southern Hemisphere, Chile (ESO Programs 076.A-0156, 078.D-0048, 080.A0516, and 082.A-0526). massive $\left(>8 M_{\odot}\right)$ stars, which have retained a significant part of their hydrogen envelope at the time of explosion. Red supergiant (RSG) stars have been found at the position of SN II explosion sites in pre-explosion images (e.g., Van Dyk et al. 2003; Smartt et al. 2004, 2009; Maund \& Smartt 2005; Smartt 2015), suggesting that they are the direct progenitors of the vast majority of SNe II.

Initially, SNe II were classified according to the shape of the light curve: SNe with faster "linear" declining light curves were cataloged as SNe IIL, while SNe with a plateau (quasi-constant luminosity for a period of a few months) as SNe IIP (Barbon 
et al. 1979). Years later, two spectroscopic classes and one photometric were added within the SNe II group: SNe IIn and SNe IIb, and SN 1987A-like, respectively. SNe IIn show longlasting narrow emission lines in their spectra (Schlegel 1990), attributed to interaction with the circumstellar medium (CSM), while $\mathrm{SNe}$ IIb are thought to be transitional objects, between SNe II and SNe Ib (Filippenko et al. 1993). On the other hand, the 1987A-like events, following the prototype of SN 1987A (e.g., Blanco et al. 1987; Menzies et al. 1987; Hamuy et al. 1988; Phillips et al. 1988; Suntzeff et al. 1988), are spectroscopically similar to the typical SNe II; however, their light curves display a peculiar long rise to maximum ( $\sim 100$ days), which is consistent with a compact progenitor. The latter three subtypes (IIn, IIb, and 87A-like) are not included in the bulk of the analysis for this paper.

Although it has been shown that $\mathrm{SNe} \mathrm{II}^{21}$ are a continuous single population (e.g., Anderson et al. 2014b; Sanders et al. 2015; Valenti et al. 2016), a large spectral and photometric diversity is observed. Pastorello et al. (2004) and Spiro et al. (2014) studied a sample of low luminosity SNe II. They show that these events present, in addition to low luminosities $\left(M_{\mathrm{V}} \geqslant-15.5\right.$ at peak), narrow spectral lines. Later, Inserra et al. (2013) analyzed a sample of moderately luminous SNe II, finding that these $\mathrm{SNe}$, in contrast to the low luminosity events, are relatively bright at peak $\left(M_{\mathrm{V}} \leqslant-16.95\right)$.

In addition to these samples, many individual studies have been published showing spectral line identification, evolution, and parameters such as velocities and pseudo-equivalent widths (pEWs) for specific SNe. Examples of very well studied SNe include SN 1979C (e.g., Branch et al. 1981; Immler et al. 2005), SN 1980K (e.g., Buta 1982; Dwek 1983; Fesen et al. 1999), SN 1999em (e.g., Baron et al. 2000; Hamuy et al. 2001; Leonard et al. 2002b; Dessart \& Hillier 2006), SN 1999gi (e.g., Leonard et al. 2002a), SN 2004et (e.g., Li et al. 2005; Sahu et al. 2006; Misra et al. 2007; Maguire et al. 2010), SN 2005cs (e.g., Pastorello et al. 2006; Dessart et al. 2008; Pastorello et al. 2009), and SN 2012aw (e.g., Bose et al. 2013; Dall'Ora et al. 2014; Jerkstrand et al. 2014). The first two SNe (1979C and 1980K) are the prototypes of fast declining SNe II (SNe IIL), together with unusually bright light curves and high ejecta velocities. On the other hand, the rest of the objects listed are generally referred to as SNe IIP, as they display relatively slowly declining light curves. For faint $\mathrm{SNe}$, similar to SN 2005cs, the expansion velocity and luminosity are even lower, probably due to low energy explosions (see Pastorello et al. 2009).

In recent years, the number of studies of individual SNe II has continued to increase; however, there are still only a handful of statistical analyses of large samples (e.g., Patat et al. 1994; Arcavi et al. 2010; Anderson et al. 2014b; Faran et al. 2014b, 2014a; Gutiérrez et al. 2014; Pejcha \& Prieto 2015a, 2015b; Sanders et al. 2015; Galbany et al. 2016; Müller et al. 2017; Valenti et al. 2016). Here we attempt to remedy this situation. The purpose of this paper is to present a statistical characterization of the optical spectra of $\mathrm{SNe}$ II, as well as an initial analysis of their spectral features. We have analyzed 888 spectra of 122 SNe II ranging between

\footnotetext{
21 Throughout the remainder of the manuscript, we use SN II to refer to all SNe that would historically have been classified as SN IIP or SN IIL. In general, we will differentiate these events by referring to their specific light curve or spectral morphology, and we only return to this historical separation if clarification and comparison with previous works is required.
}

3 and 363 days since explosion. We selected 11 features in the photospheric phase with the aim of understanding the overall evolution of visual-wavelength spectroscopy of $\mathrm{SNe}$ II with time.

The paper is organized as follows. In Section 2, we describe the data sample. The spectroscopic observations and data reduction techniques are presented in Section 3. In Section 4, the estimation of the explosion epoch is presented. In Section 5, we describe the sample properties, while in Section 6 we identify spectral features. The spectral measurements are presented in Section 7, while the line evolution analysis and the conclusions are in Sections 8 and 9, respectively.

In Paper II, we study the correlations between different spectral and photometric parameters, and try to understand these in terms of the diversity of the underlying physics of the explosions and their progenitors.

\section{Data Sample}

Our data set was obtained between 1986 and 2009 from a variety of different sources. This sample consists of 888 optical spectra of $122 \mathrm{SNe}$ II, ${ }^{22}$ of which 4 were provided by the Cerro Tololo Supernova Survey (CTSS), 7 were obtained by the Calán/Tololo survey (CT, Hamuy et al. 1993, PI: Hamuy 1989-1993), 5 by the Supernova Optical and Infrared Survey (SOIRS, PI: Hamuy, 1999-2000), 31 by the Carnegie Type II Supernova Survey (CATS, PI: Hamuy, 2002-2003), and 75 by the Carnegie Supernova Project (CSP-I, Hamuy et al. 2006, 2004-2009). These follow-up campaigns concentrated on obtaining well-sampled and high-cadence light curves and spectral sequences of nearby $\mathrm{SNe}$, based mainly on two criteria: (1) that the SN was brighter than $V \sim 17$ mag at discovery and (2) that those discovered $\mathrm{SNe}$ were classified as being relatively young, i.e., less than one month from explosion.

The redshift distribution of our sample is shown in Figure 1. The figure shows that the majority of the sample has a redshift $\leqslant 0.03$. SN 2002ig has the highest redshift in the sample with a value of 0.077 , while the nearest SN (SN 2008bk) has a redshift of 0.00076 . The mean redshift value of the sample is 0.0179 and the median is 0.0152 . The redshift information comes from the heliocentric recession velocity of each host galaxy as published in the NASA/IPAC extragalactic Database (NED). ${ }^{23}$ These NED values were compared with those obtained through the measurement of narrow emission lines observed within SN spectra and originating from host $\mathrm{H}$ II regions. In cases of discrepancy between the two sources, we give priority to our spectral estimations. Two of our objects (SN 2006Y and SN 2007ld) occur in unknown host galaxies. Their redshifts were obtained from the Asiago supernova catalog ${ }^{24}$ and from the narrow emission lines within SN spectra originating from the underlying host galaxy, respectively. Table 1 lists the sample of SNe II selected for this work, their host galaxy information, and the campaign to which they belong.

From our SNe II sample, SNe IIn-, SNe IIb-, and SN 1987A-like events (SN 2006au and SN 2006V; Taddia et al. 2012) were excluded based on photometric information. Details of the SNe IIn sample can be found in Taddia et al. (2013), while those of the SNe IIb in Stritzinger et al. (2017)

\footnotetext{
22 In the data release, we include eight spectra of the SN 2000cb, an SN 1987A-like event, which is not analyzed in this work.

23 http://ned.ipac.caltech.edu

24 http://sngroup.oapd.inaf.it
} 


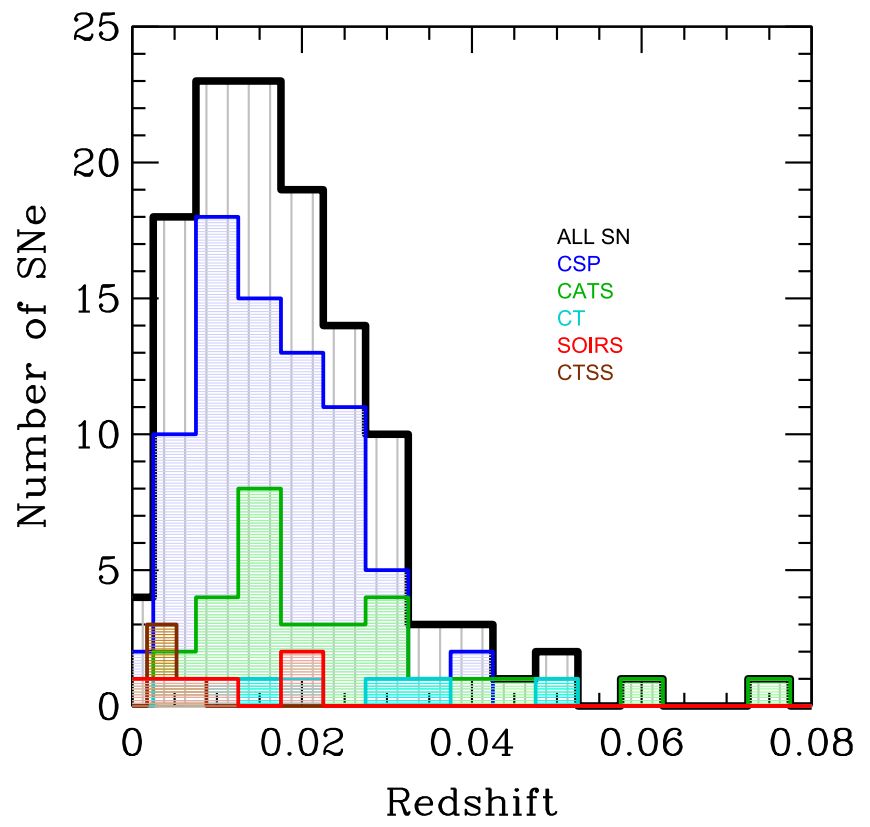

Figure 1. Distribution of heliocentric redshifts for the 122 SN II in our sample.

and Taddia et al. (2017). The photometry of our sample in the $V$ band was published by Anderson et al. (2014b). More recently, Galbany et al. (2016) released the UBVRIz photometry of our sample obtained by CATS between 1986 and 2003. Around 750 spectra of $\sim 100$ objects are published here for the first time. Now we briefly discuss each of the surveys providing $\mathrm{SNe}$ for our analysis.

\subsection{The Cerro Tololo Supernova Survey}

A total of four SNe II (SN 1986L, SN 1988A, SN 1990E, and SN 1990K) were extensively observed at CTIO by the Cerro Tololo SN program (PIs: Phillips and Suntzeff, 1986-2003). These SNe have been analyzed in previous works (e.g Schmidt et al. 1993; Turatto et al. 1993; Cappellaro et al. 1995; Hamuy 2001).

\subsection{The Calán/Tololo Survey (CT)}

The Calán/Tololo survey was a program of both discovery and follow-up of SNe. A total of $50 \mathrm{SNe}$ were obtained between 1989 and 1993. The analysis of SNe Ia was published by Hamuy et al. (1996). Spectral and photometric details of six SNe II were presented by Hamuy (2001). In this analysis, we include these SNe II and an additional object, SN 1993K.

\subsection{The Supernova Optical and Infrared Survey (SOIRS)}

The Supernova Optical and Infrared Survey carried out a program to obtain optical and IR photometry and spectroscopy of nearby SNe $(z<0.08)$. In the course of 1999-2000, $20 \mathrm{SNe}$ were observed, 6 of which are SNe II. Details of these SNe were published by Hamuy (2001, 2003), Hamuy et al. (2001), and Hamuy \& Pinto (2002).

\subsection{The Carnegie Type II Supernova Survey (CATS)}

Between 2002 and 2003 the Carnegie Type II Supernova Survey observed 34 SNe II. While optical spectroscopy and photometry of these $\mathrm{SNe}$ II have been previously used to derive distances (Olivares 2008; Jones et al. 2009), the spectral observations have not been officially released until now.

\subsection{The Carnegie Supernova Project I (CSP-I)}

The Carnegie Supernova Project I (CSP-I) was a five-year follow-up program to obtain high quality optical and nearinfrared light curves and optical spectroscopy. The data obtained by the CSP-I between 2004 and 2009 consist of $\sim 250 \mathrm{SNe}$ of all types, of which 75 correspond to SNe II. The first SN Ia photometry data were published in Contreras et al. (2010), while their analysis was done by Folatelli et al. (2010). A second data release was provided by Stritzinger et al. (2011). A spectroscopy analysis of SNe Ia was published by Folatelli et al. (2013). Recently, Stritzinger et al. (2017) and Taddia et al. (2017) published the photometry data release of strippedenvelope supernovae. The CSP-I spectral data for SNe II are published here for the first time, while the complete optical and near-IR photometry will be published by C. Contreras et al. (2017, in preparation).

\section{Observations and Data Reduction}

In this section, we summarize our observations and the data reduction techniques. However, a detailed description of the CT methodology is presented in Hamuy et al. (1993), for the case of SOIRS the CT methodology is described in Hamuy et al. (2001), and for CSP-I it can be found in Hamuy et al. (2006) and Folatelli et al. (2013).

\subsection{Observations}

The data presented here were obtained with a large variety of instruments and telescopes, as shown in Table 6. The majority of the spectra were taken in long-slit spectroscopic mode with the slit placed along the parallactic angle. However, when the SN was located close to the host, it was necessary to pick a different and more convenient angle to avoid contamination from the host. The majority of our spectra cover the range of $\sim 3800$ to $\sim 9500 \AA$. The observations were performed with the Cassegrain spectrographs at $1.5 \mathrm{~m}$ and $4.0 \mathrm{~m}$ telescopes at Cerro Tololo, with the Wide Field CCD Camera (WFCCD) at the $2.5 \mathrm{~m}$ du Pont Telescope, the Low Dispersion Survey Spectrograph (LDSS2; Allington-Smith et al. 1994) on the Magellan Clay $6.5 \mathrm{~m}$ telescope and the Inamori Magellan Areal Camera and Spectrograph (IMACS; Dressler et al. 2011) on the Magellan Baade $6.5 \mathrm{~m}$ telescope at Las Campanas Observatory. At La Silla, the observations were carried out with the ESO Multi-Mode Instrument (EMMI; Dekker et al. 1986) in medium resolution spectroscopy mode (at the NTT) and the ESO Faint Object Spectrograph and Camera (EFOSC; Buzzoni et al. 1984) at the NTT and $3.6 \mathrm{~m}$ telescopes. We also have three spectra for SN 2006ee obtained with the Boller \& Chivens CCD spectrograph at the Hiltner $2.4 \mathrm{~m}$ Telescope of the MDM Observatory. Table 6 displays a complete journal of the 888 spectral observations, listing for each spectrum the UT and Julian dates, phases, wavelength range, FWHM resolution, exposure time, airmass, and the telescope and instrument used.

The distribution of the number of spectra per object for our sample is shown in Figure 2. Seven SNe (SN 1993A, SN 2005dt, SN 2005dx, SN 2005es, SN 2005gz, SN 2005me, and SN 2008H) only have one spectrum, while $90 \%$ of the sample have between 2 and 12 spectra. SN 1986L is the object 
Table 1

SN II Sample

\begin{tabular}{|c|c|c|c|c|c|c|c|c|c|}
\hline SN & $\begin{array}{l}\text { Host } \\
\text { Galaxy }\end{array}$ & $\begin{array}{c}\text { Recession } \\
\text { Velocity }\left(\mathrm{km} \mathrm{s}^{-1}\right)\end{array}$ & $\begin{array}{l}\text { Hubble } \\
\text { Type }\end{array}$ & $\begin{array}{c}E(B-V)_{\mathrm{MW}} \\
(\mathrm{mag})\end{array}$ & $\begin{array}{l}\text { Discovery } \\
\text { Date }\end{array}$ & $\begin{array}{l}\text { Discovery } \\
\text { Reference }\end{array}$ & $\begin{array}{l}\text { Explosion } \\
\text { Epoch }\end{array}$ & $\begin{array}{l}\mathrm{N} \text { of } \\
\text { Spectra }\end{array}$ & Campaign \\
\hline $1986 \mathrm{~L}$ & NGC 1559 & 1305 & SBcd & 0.026 & 46711.1 & IAUC 4260 & $46708.0^{\mathrm{a}}(3)$ & 31 & CTSS \\
\hline 1988A & NGC 4579 & 1517 & $\mathrm{SABb}$ & 0.036 & 47179.0 & IAUC 4533 & $47177.2^{\mathrm{a}}(2)$ & 5 & CTSS \\
\hline $1990 \mathrm{E}$ & NGC 1035 & 1241 & SAc & 0.022 & 47937.7 & IAUC 4965 & $47935.1^{\mathrm{a}}(3)$ & 5 & CTSS \\
\hline $1990 \mathrm{~K}$ & NGC 0150 & 1584 & $\mathrm{SBbc}$ & 0.013 & 48037.3 & IAUC 5022 & $48001.5^{\mathrm{a}}(6)$ & 9 & CTSS \\
\hline 1991al & 2MASX J19422191-5506275 & $4575^{\mathrm{b}}$ & $?$ & 0.054 & 48453.7 & IAUC 5310 & $48442.5^{\mathrm{c}}(8)^{\mathrm{d}}$ & 8 & $\mathrm{CT}$ \\
\hline 1992af & ESO $340-G 038$ & 5541 & S & 0.046 & 48802.8 & IAUC 5554 & $48798.8^{\mathrm{c}}(8)^{\mathrm{d}}$ & 5 & $\mathrm{CT}$ \\
\hline 1992am & MCG -01-04-039 & $14397^{\mathrm{b}}$ & S & 0.046 & 48829.8 & IAUC 5570 & $48813.9^{c}(6)^{d}$ & 2 & $\mathrm{CT}$ \\
\hline 1992ba & NGC 2082 & 1185 & $\mathrm{SABc}$ & 0.051 & 48896.2 & IAUC 5625 & $48884.9^{\mathrm{c}}(7)$ & 10 & $\mathrm{CT}$ \\
\hline 1993A & 2MASX J07391822-6203095 & $8790^{\mathrm{b}}$ & $?$ & 0.153 & 49004.6 & IAUC 5693 & $48995.5^{\mathrm{a}}(9)$ & 2 & $\mathrm{CT}$ \\
\hline $1993 K$ & NGC 2223 & 2724 & SBbc & 0.056 & 49075.5 & IAUC 5733 & $49065.5^{\mathrm{a}}(9)$ & 17 & $\mathrm{CT}$ \\
\hline $1993 \mathrm{~S}$ & 2MASX J22522390-4018432 & 9903 & $\mathrm{~S}$ & 0.014 & 49133.7 & IAUC 5812 & $49130.8^{\mathrm{c}}(5)$ & 4 & $\mathrm{CT}$ \\
\hline $1999 \mathrm{br}$ & NGC 4900 & 960 & $\mathrm{SBc}$ & 0.021 & 51281.0 & IAUC 7141 & $51276.7^{\mathrm{a}}(4)$ & 8 & SOIRS \\
\hline 1999ca & NGC 3120 & 2793 & $\mathrm{Sc}$ & 0.096 & 51296.0 & IAUC 7158 & $51277.5^{\mathrm{c}}(7)^{\mathrm{d}}$ & 4 & SOIRS \\
\hline $1999 \mathrm{cr}$ & ESO 576-G034 & $6069^{\mathrm{b}}$ & $\mathrm{S} / \mathrm{Irr}$ & 0.086 & 51249.7 & IAUC 7210 & $51246.5^{c}(4)^{d}$ & 5 & SOIRS \\
\hline 1999 eg & IC 1861 & 6708 & SA0 & 0.104 & 51455.5 & IAUC 7275 & $51449.5^{c}(6)^{d}$ & 2 & SOIRS \\
\hline $1999 \mathrm{em}$ & NGC 1637 & 717 & $\mathrm{SABc}$ & 0.036 & 51481.0 & IAUC 7294 & $51476.5^{\mathrm{a}}(5)$ & 12 & SOIRS \\
\hline $2002 \mathrm{ew}$ & NEAT J205430.50-000822.0 & 8975 & $?$ & 0.091 & 52510.8 & IAUC 7964 & $52500.6^{\mathrm{a}}(10)$ & 7 & CATS \\
\hline $2002 \mathrm{fa}$ & NEAT J205221.51 + 020841.9 & 17988 & $?$ & 0.088 & 52510.8 & IAUC 7967 & $52502.5^{\mathrm{c}}(8)^{\mathrm{d}}$ & 6 & CATS \\
\hline $2002 \mathrm{gd}$ & NGC 7537 & 2676 & $\mathrm{SAbc}$ & 0.059 & 52552.7 & IAUC 7986 & $52551.5^{\mathrm{c}}(4)^{\mathrm{d}}$ & 12 & CATS \\
\hline $2002 \mathrm{gw}$ & NGC 922 & 3084 & SBcd & 0.017 & 52560.7 & IAUC 7995 & $52553.5^{\mathrm{c}}(8)^{\mathrm{d}}$ & 11 & CATS \\
\hline $2002 \mathrm{hj}$ & NPM1G +04.0097 & 7080 & $?$ & 0.102 & 52568.0 & IAUC 8006 & $52562.5^{\mathrm{a}}(7)$ & 7 & CATS \\
\hline $2002 \mathrm{hx}$ & PGC 023727 & 9293 & $\mathrm{SBb}$ & 0.048 & 52589.7 & IAUC 8015 & $52582.5^{\mathrm{a}}(9)$ & 9 & CATS \\
\hline $2002 \mathrm{ig}$ & SDSS J013637.22 + 005524.9 & $23100^{\mathrm{e}}$ & $?$ & 0.034 & 52576.7 & IAUC 8020 & $52570.5^{c}(5)^{d}$ & 5 & CATS \\
\hline 210 & MCG +00-03-054 & 15420 & $?$ & 0.033 & $?^{\mathrm{f}}$ & ? & $52486.5^{\mathrm{c}}(6)^{\mathrm{d}}$ & 6 & CATS \\
\hline 2003B & NGC 1097 & 1272 & $\mathrm{SBb}$ & 0.024 & 52645.0 & IAUC 8042 & $52613.5^{\mathrm{c}}(11)^{\mathrm{d}}$ & 9 & CATS \\
\hline $2003 \mathrm{E}$ & MCG -4-12-004 & $4470^{\mathrm{b}}$ & $\mathrm{Sbc}$ & 0.043 & 52645.0 & IAUC 8044 & $52629.5^{\mathrm{c}}(8)^{\mathrm{d}}$ & 8 & CATS \\
\hline $2003 \mathrm{~T}$ & UGC 4864 & 8373 & SAab & 0.028 & 52665.0 & IAUC 8058 & $52654.5^{\mathrm{a}}(10)$ & 6 & CATS \\
\hline $2003 b l$ & NGC 5374 & $4377^{\mathrm{b}}$ & SBbc & 0.024 & 52701.0 & IAUC 8086 & $52696.5^{c}(4)^{d}$ & 8 & CATS \\
\hline 2003bn & 2MASX J10023529-2110531 & 3828 & $?$ & 0.057 & 52698.0 & IAUC 8088 & $52694.5^{\mathrm{a}}(3)$ & 12 & CATS \\
\hline $2003 \mathrm{ci}$ & UGC 6212 & 9111 & $\mathrm{Sb}$ & 0.053 & 52720.0 & IAUC 8097 & $52711.5^{\mathrm{a}}(8)$ & 7 & CATS \\
\hline $2003 \mathrm{cn}$ & IC 849 & $5433^{\mathrm{b}}$ & SABcd & 0.019 & 52728.0 & IAUC 8101 & $52717.5^{\mathrm{c}}(4)^{\mathrm{d}}$ & 5 & CATS \\
\hline $2003 c x$ & NEAT J135706.53-170220.0 & 11100 & $?$ & 0.083 & 52730.0 & IAUC 8105 & $52725.5^{\mathrm{c}}(5)^{\mathrm{d}}$ & 6 & CATS \\
\hline $2003 \mathrm{dq}$ & MAPS-NGP O4320786358 & 13800 & $?$ & 0.016 & 52739.7 & IAUC 8117 & $52731.5^{\mathrm{a}}(8)$ & 3 & CATS \\
\hline $2003 \mathrm{ef}$ & NGC 4708 & $4440^{\mathrm{b}}$ & SAab & 0.041 & 52770.7 & IAUC 8131 & $52757.5^{\mathrm{c}}(9)^{\mathrm{d}}$ & 6 & CATS \\
\hline 2003eg & NGC 4727 & $4388^{\mathrm{b}}$ & SABbc & 0.046 & 52776.7 & IAUC 8134 & $52764.5^{\mathrm{c}}(5)^{\mathrm{d}}$ & 5 & CATS \\
\hline 2003ej & UGC 7820 & 5094 & SABcd & 0.017 & 52779.7 & IAUC 8134 & $52775.5^{\mathrm{a}}(5)$ & 3 & CATS \\
\hline $2003 \mathrm{fb}$ & UGC 11522 & $5262^{\mathrm{b}}$ & $\mathrm{Sbc}$ & 0.162 & 52796.0 & IAUC 8143 & $52772.5^{\mathrm{c}}(10)^{\mathrm{d}}$ & 4 & CATS \\
\hline $2003 \mathrm{gd}$ & M74 & 657 & SAc & 0.062 & 52803.2 & IAUC 8150 & $52755.5^{\mathrm{c}}(9)^{\mathrm{d}}$ & 3 & CATS \\
\hline 2003hd & MCG -04-05-010 & 11850 & $\mathrm{Sb}$ & 0.011 & 52861.0 & IAUC 8179 & $52855.9^{c}(5)^{d}$ & 9 & CATS \\
\hline 2003hg & NGC 7771 & 4281 & $\mathrm{SBa}$ & 0.065 & 52870.0 & IAUC 8184 & $52865.5^{\mathrm{a}}(5)$ & 5 & CATS \\
\hline $2003 \mathrm{hk}$ & NGC 1085 & 6795 & SAbc & 0.033 & 52871.6 & CBET 41 & $52866.8^{\mathrm{c}}(4)^{\mathrm{d}}$ & 4 & CATS \\
\hline $2003 \mathrm{hl}$ & NGC 772 & 2475 & $\mathrm{SAb}$ & 0.064 & 52872.0 & IAUC 8184 & $52868.5^{\mathrm{a}(5)}$ & 6 & CATS \\
\hline $2003 \mathrm{hn}$ & NGC 1448 & 1170 & SAcd & 0.013 & 52877.2 & IAUC 8186 & $52866.5^{\mathrm{a}}(10)$ & 9 & CATS \\
\hline 2003 ho & ESO 235-G58 & 4314 & SBcd & 0.034 & 52851.9 & IAUC 8186 & $52848.5^{\mathrm{c}}(7)^{\mathrm{d}}$ & 5 & CATS \\
\hline $2003 i b$ & MCG -04-48-15 & 7446 & $\mathrm{Sb}$ & 0.043 & 52898.7 & IAUC 8201 & $52891.5^{\mathrm{a}}(8)$ & 5 & CATS \\
\hline 2003ip & UGC 327 & 5403 & $\mathrm{Sbc}$ & 0.058 & 52913.7 & IAUC 8214 & $52896.5^{\mathrm{c}}(4)$ & 4 & CATS \\
\hline $2003 i q$ & NGC 772 & 2475 & $\mathrm{SAb}$ & 0.064 & 52921.5 & CBET 48 & $52919.5^{\mathrm{a}}(2)$ & 5 & CATS \\
\hline 2004dy & IC 5090 & 9352 & $\mathrm{Sa}$ & 0.045 & 53242.5 & IAUC 8395 & $53240.5^{\mathrm{a}}(2)$ & 3 & CSP \\
\hline 2004ej & NGC 3095 & 2723 & $\mathrm{SBc}$ & 0.061 & 53258.5 & CBET 78 & $53223.9^{c}(9)^{d}$ & 9 & CSP \\
\hline $2004 \mathrm{er}$ & MCG -01-7-24 & 4411 & SAc & 0.023 & 53274.0 & CBET 93 & $53271.8^{\mathrm{a}}(2)$ & 10 & CSP \\
\hline $2004 \mathrm{fb}$ & ESO $340-G 7$ & 6100 & $\mathrm{~S}$ & 0.056 & 53286.2 & IAUC 8420 & $53258.6^{c}(7)^{d}$ & 4 & CSP \\
\hline $2004 \mathrm{fc}$ & NGC 701 & 1831 & $\mathrm{SBc}$ & 0.023 & 53295.2 & IAUC 8422 & $53293.5^{\mathrm{a}}(1)$ & 10 & CSP \\
\hline $2004 f x$ & MCG -02-14-3 & 2673 & $\mathrm{SBc}$ & 0.090 & 53307.0 & IAUC 8431 & $53303.5^{\mathrm{a}}(4)$ & 10 & CSP \\
\hline $2005 \mathrm{~J}$ & NGC 4012 & 4183 & $\mathrm{Sb}$ & 0.025 & 53387.0 & IAUC 8467 & $53379.8^{\mathrm{c}}(7)^{\mathrm{d}}$ & 11 & CSP \\
\hline $2005 \mathrm{~K}$ & NGC 2923 & 8204 & $?$ & 0.035 & 53386.0 & IAUC 8468 & $53369.8^{\mathrm{c}}(8)$ & 2 & CSP \\
\hline $2005 Z$ & NGC 3363 & 5766 & $\mathrm{~S}$ & 0.025 & 53402.0 & IAUC 8476 & $53396.7^{\mathrm{a}}(6)$ & 9 & CSP \\
\hline 2005af & NGC 4945 & 563 & SBcd & 0.156 & 53409.7 & IAUC 8482 & $53320.8^{\mathrm{c}}(17)^{\mathrm{d}}$ & 9 & CSP \\
\hline 2005an & ESO 506-G11 & 3206 & So & 0.083 & 53432.7 & CBET 113 & $53431.8^{\mathrm{c}}(6)^{\mathrm{d}}$ & 7 & CSP \\
\hline $2005 \mathrm{dk}$ & IC 4882 & 4708 & $\mathrm{SBb}$ & 0.043 & 53604.0 & IAUC 8586 & $53601.5^{c}(6)^{d}$ & 7 & CSP \\
\hline
\end{tabular}


Table 1

(Continued)

\begin{tabular}{|c|c|c|c|c|c|c|c|c|c|}
\hline SN & $\begin{array}{l}\text { Host } \\
\text { Galaxy }\end{array}$ & $\begin{array}{c}\text { Recession } \\
\text { Velocity }\left(\mathrm{km} \mathrm{s}^{-1}\right)\end{array}$ & $\begin{array}{l}\text { Hubble } \\
\text { Type }\end{array}$ & $\begin{array}{c}E(B-V)_{\mathrm{MW}} \\
(\mathrm{mag})\end{array}$ & $\begin{array}{l}\text { Discovery } \\
\text { Date }\end{array}$ & $\begin{array}{l}\text { Discovery } \\
\text { Reference }\end{array}$ & $\begin{array}{l}\text { Explosion } \\
\text { Epoch }\end{array}$ & $\begin{array}{l}\mathrm{N} \text { of } \\
\text { Spectra }\end{array}$ & Campaign \\
\hline $2005 \mathrm{dn}$ & NGC 6861 & 2829 & SA0 & 0.048 & 53609.5 & IAUC 8589 & $53602.6^{\mathrm{c}}(6)^{\mathrm{d}}$ & 8 & CSP \\
\hline $2005 \mathrm{dt}$ & MCG -03-59-6 & 7695 & $\mathrm{SBb}$ & 0.025 & 53614.7 & CBET 213 & $53605.6^{\mathrm{a}}(9)$ & 1 & CSP \\
\hline $2005 \mathrm{dw}$ & MCG -05-52-49 & 5269 & $\mathrm{Sab}$ & 0.020 & 53612.7 & CBET 219 & $53603.6^{\mathrm{a}}(9)$ & 3 & CSP \\
\hline $2005 d x$ & MCG -03-11-9 & 8012 & $\mathrm{~S}$ & 0.021 & 53623.0 & CBET 220 & $53611.8^{\mathrm{c}}(7)^{\mathrm{d}}$ & 1 & CSP \\
\hline $2005 \mathrm{dz}$ & UGC 12717 & 5696 & Scd & 0.072 & 53623.7 & CBET 222 & $53619.5^{\mathrm{a}}(4)$ & 7 & CSP \\
\hline 2005es & MCG +01-59-79 & 11287 & S & 0.076 & 53643.7 & IAUC 8608 & $53638.7^{\mathrm{a}}(5)$ & 1 & CSP \\
\hline $2005 \mathrm{gz}$ & MCG -01-53-022 & 8518 & $\mathrm{SBbc}$ & 0.06 & 53654.7 & IAUC 8616 & $53650.2^{\mathrm{a}}(5)$ & 1 & CSP \\
\hline 2005lw & IC 672 & 7710 & $?$ & 0.043 & 53719.0 & CBET 318 & $53716.8^{\mathrm{c}}(10)$ & 14 & CSP \\
\hline $2005 \mathrm{me}$ & ESO 244-31 & 6726 & SAc & 0.022 & 53728.2 & CBET 333 & $53717.9^{\mathrm{c}}(10)^{\mathrm{d}}$ & 1 & CSP \\
\hline 2006Y & anon & $10074^{\mathrm{e}}$ & $?$ & 0.115 & 53770.0 & IAUC 8668 & $53766.5^{\mathrm{a}}(4)$ & 13 & CSP \\
\hline 2006ai & ESO 005-G009 & $4571^{\mathrm{b}}$ & SBcd & 0.113 & 53784.0 & CBET 406 & $53781.6^{\mathrm{c}}(5)$ & 12 & CSP \\
\hline $2006 \mathrm{bc}$ & NGC 2397 & 1363 & $\mathrm{SABb}$ & 0.181 & 53819.1 & CBET 446 & $53815.5^{\mathrm{a}}(4)$ & 3 & CSP \\
\hline 2006be & IC 4582 & 2145 & S & 0.026 & 53819.0 & CBET 449 & $53802.8^{\mathrm{c}}(9)^{\mathrm{d}}$ & 4 & CSP \\
\hline $2006 \mathrm{bl}$ & MCG +02-40-9 & 9708 & $?$ & 0.045 & 53829.5 & CBET 597 & $53822.7^{\mathrm{c}}(10)^{\mathrm{d}}$ & 3 & CSP \\
\hline 2006ee & NGC 774 & 4620 & So & 0.054 & 53966.0 & cbet 597 & $53961.9^{\mathrm{a}}(4)$ & 13 & CSP \\
\hline 2006it & NGC 6956 & 4650 & $\mathrm{SBb}$ & 0.087 & 54009.5 & CBET 660 & $54006.5^{\mathrm{a}}(3)$ & 6 & CSP \\
\hline 2006iw & 2MASX J23211915 + 0015329 & 9226 & $?$ & 0.044 & 54011.5 & CBET 663 & $54010.7^{\mathrm{a}}(1)$ & 5 & CSP \\
\hline $2006 \mathrm{~ms}$ & NGC 6935 & 4543 & SAa & 0.031 & 54046.2 & CBET 725 & $54028.5^{\mathrm{c}}(6)^{* *}$ & 4 & CSP \\
\hline 2006qr & MCG -02-22-023 & 4350 & SABbc & 0.040 & 54070.0 & CBET 766 & $54062.8^{\mathrm{a}}(7)$ & 8 & CSP \\
\hline 2007P & ESO 566-G36 & 12224 & $\mathrm{Sa}$ & 0.036 & 54124.0 & CBET 819 & $54118.7^{\mathrm{a}}(5)$ & 6 & CSP \\
\hline $2007 \mathrm{U}$ & ESO 552-65 & 7791 & $\mathrm{~S}$ & 0.046 & 54136.5 & CBET 835 & $54133.6^{\mathrm{c}}(6)^{\mathrm{d}}$ & 7 & CSP \\
\hline $2007 \mathrm{~W}$ & NGC 5105 & 2902 & $\mathrm{SBc}$ & 0.045 & 54146.5 & CBET 844 & $54130.8^{\mathrm{c}}(7)^{\mathrm{d}}$ & 7 & CSP \\
\hline $2007 X$ & ESO 385-G32 & 2837 & $\mathrm{SABc}$ & 0.060 & 54146.5 & CBET 844 & $54143.5^{\mathrm{c}}(5)$ & 12 & CSP \\
\hline $2007 Z$ & PGC 016993 & 5277 & $\mathrm{Sbc}$ & 0.525 & 54148.7 & CBET 847 & $54135.6^{\mathrm{c}}(5)$ & 2 & CSP \\
\hline $2007 \mathrm{aa}$ & NGC 4030 & 1465 & SAbc & 0.023 & 54149.7 & CBET 848 & $54126.7^{\mathrm{c}}(8)^{\mathrm{d}}$ & 11 & CSP \\
\hline $2007 \mathrm{ab}$ & MCG -01-43-2 & 7056 & SBbc & 0.235 & 54150.7 & CBET 851 & $54123.9^{\mathrm{c}}(10)$ & 5 & CSP \\
\hline $2007 \mathrm{av}$ & NGC 3279 & 1394 & Scd & 0.032 & 54180.2 & CBET 901 & $54173.8^{\mathrm{c}}(5)^{\mathrm{d}}$ & 4 & CSP \\
\hline 2007bf & UGC 09121 & 5327 & $\mathrm{Sbc}$ & 0.018 & 54285.0 & CBET 919 & $54191.5^{\mathrm{a}}(7)$ & 4 & CSP \\
\hline $2007 \mathrm{hm}$ & SDSS J205755.65-072324.9 & 7540 & $?$ & 0.059 & 54343.7 & CBET 1050 & $54336.6^{c}(6)^{d}$ & 7 & $\mathrm{CSP}$ \\
\hline 2007il & IC 1704 & 6454 & $\mathrm{~S}$ & 0.042 & 54354.0 & CBET 1062 & $54349.8^{\mathrm{a}}(4)$ & 12 & CSP \\
\hline 2007it & NGC 5530 & 1193 & SAc & 0.103 & 54357.5 & CBET 1065 & $54348.5^{\mathrm{a}}(1)$ & 11 & CSP \\
\hline 2007ld & anon & $7499^{\mathrm{b}}$ & $?$ & 0.081 & 54379.5 & CBET 1098 & $54376.5^{\mathrm{c}}(8)^{\mathrm{d}}$ & 7 & CSP \\
\hline 2007 oc & NGC 7418 & 1450 & SABcd & 0.014 & 54396.5 & CBET 1114 & $54388.5^{\mathrm{a}}(3)$ & 17 & CSP \\
\hline 2007od & UGC 12846 & 1734 & $\mathrm{Sm}$ & 0.032 & 54407.2 & CBET 1116 & $54400.6^{\mathrm{c}}(5)^{\mathrm{d}}$ & 14 & CSP \\
\hline $2007 \mathrm{sq}$ & MCG -03-23-5 & 4579 & SAbc & 0.183 & 54443.0 & CBET 1170 & $54422.8^{\mathrm{c}}(6)^{\mathrm{d}}$ & 7 & CSP \\
\hline $2008 \mathrm{~F}$ & MCG -01-8-15 & 5506 & $\mathrm{SBa}$ & 0.044 & 54477.5 & CBET 1207 & $54469.6^{\mathrm{c}}(6)^{\mathrm{d}}$ & 2 & CSP \\
\hline $2008 \mathrm{H}$ & ESO 499- G 005 & 4287 & SAc & 0.057 & 54481.0 & CBET 1210 & $54432.8^{\mathrm{c}}(8)$ & 1 & CSP \\
\hline $2008 \mathrm{~K}$ & ESO 504-G5 & 7997 & $\mathrm{Sb}$ & 0.035 & 54481.0 & CBET 1211 & $54475.5^{\mathrm{c}}(6)^{\mathrm{d}}$ & 12 & CSP \\
\hline $2008 \mathrm{M}$ & ESO 121-26 & 2267 & $\mathrm{SBc}$ & 0.040 & 54480.7 & CBET 1214 & $54471.7^{\mathrm{a}}(9)$ & 12 & CSP \\
\hline 2008W & MCG -03-22-7 & 5757 & $\mathrm{Sc}$ & 0.086 & 54502.7 & CBET 1238 & $54483.8^{\mathrm{c}}(8)^{\mathrm{d}}$ & 10 & $\mathrm{CSP}$ \\
\hline 2008ag & IC 4729 & 4439 & $\mathrm{SABbc}$ & 0.074 & 54499.5 & CBET 1252 & $54477.9^{c}(8)^{d}$ & 18 & CSP \\
\hline 2008aw & NGC 4939 & 3110 & $\mathrm{SAbc}$ & 0.036 & 54528.0 & CBET 1279 & $54517.8^{\mathrm{a}}(10)$ & 12 & CSP \\
\hline 2008bh & NGC 2642 & 4345 & SBbc & 0.020 & 54549.0 & CBET 1311 & $54543.5^{\mathrm{a}}(5)$ & 6 & CSP \\
\hline $2008 \mathrm{bk}$ & NGC 7793 & 227 & SAd & 0.017 & 54550.7 & CBET 1315 & $54540.9^{\mathrm{c}}(8)^{\mathrm{d}}$ & 26 & CSP \\
\hline $2008 \mathrm{bm}$ & CGCG 071-101 & 9563 & $\mathrm{Sc}$ & 0.023 & 54554.7 & CBET 1320 & $54522.8^{\mathrm{c}}(6)$ & 4 & CSP \\
\hline $2008 \mathrm{bp}$ & NGC 3095 & 2723 & $\mathrm{SBc}$ & 0.061 & 54558.7 & CBET 1326 & $54551.7^{\mathrm{a}}(6)$ & 5 & CSP \\
\hline $2008 \mathrm{br}$ & IC 2522 & 3019 & SAcd & 0.083 & 54564.2 & CBET 1332 & $54555.7^{\mathrm{a}}(9)$ & 4 & CSP \\
\hline 2008bu & ESO 586-G2 & 6630 & S & 0.376 & 54574.0 & CBET 1341 & $54566.8^{\mathrm{c}}(7)$ & 5 & CSP \\
\hline 2008ga & LCSB L0250N & 4639 & $?$ & 0.582 & 54734.0 & CBET 1526 & $54711.5^{\mathrm{c}}(7)$ & 3 & CSP \\
\hline $2008 \mathrm{gi}$ & CGCG 415-004 & 7328 & $\mathrm{Sc}$ & 0.060 & 54752.0 & CBET 1539 & $54742.7^{\mathrm{a}}(9)$ & 6 & CSP \\
\hline $2008 \mathrm{gr}$ & IC 1579 & 6831 & SBbc & 0.012 & 54768.7 & CBET 1557 & $54769.6^{c}(6)^{d}$ & 5 & CSP \\
\hline 2008hg & IC 1720 & 5684 & $\mathrm{Sbc}$ & 0.016 & 54785.5 & CBET 1571 & $54779.8^{\mathrm{a}}(5)$ & 6 & CSP \\
\hline 2008ho & NGC 922 & 3082 & SBcd & 0.017 & 54796.5 & CBET 1587 & $54792.7^{\mathrm{a}}(5)$ & 3 & CSP \\
\hline 2008if & MCG -01-24-10 & 3440 & $\mathrm{Sb}$ & 0.029 & 54812.7 & CBET 1619 & $54807.8^{\mathrm{a}}(5)$ & 20 & CSP \\
\hline 2008il & ESO $355-G 4$ & 6276 & $\mathrm{SBb}$ & 0.015 & 54827.7 & CBET 1634 & $54825.6^{\mathrm{a}}(3)$ & 3 & CSP \\
\hline 2008in & NGC 4303 & 1566 & $\mathrm{SABbc}$ & 0.020 & 54827.2 & CBET 1636 & $54825.4^{\mathrm{a}}(2)^{\mathrm{d}}$ & 13 & CSP \\
\hline $2009 N$ & NGC 4487 & 1034 & SABcd & 0.019 & 54856.3 & CBET 1670 & $54846.8^{\mathrm{c}}(5)$ & 13 & CSP \\
\hline 2009W & SDSS J162346.79 + 114423 & 5100 & $?$ & 0.065 & 54865.0 & CBET 1683 & $54816.9^{\mathrm{c}}(9)$ & 1 & CSP \\
\hline 2009aj & ESO 221- G 018 & 2883 & $\mathrm{Sa}$ & 0.130 & 54887.0 & CBET 1704 & $54880.5^{\mathrm{a}}(7)$ & 12 & CSP \\
\hline
\end{tabular}


Table 1

(Continued)

\begin{tabular}{|c|c|c|c|c|c|c|c|c|c|}
\hline SN & $\begin{array}{c}\text { Host } \\
\text { Galaxy }\end{array}$ & $\begin{array}{c}\text { Recession } \\
\text { Velocity }\left(\mathrm{km} \mathrm{s}^{-1}\right)\end{array}$ & $\begin{array}{l}\text { Hubble } \\
\text { Type }\end{array}$ & $\underset{(\mathrm{mag})}{E(B-V)_{\mathrm{MW}}}$ & $\begin{array}{l}\text { Discovery } \\
\text { Date }\end{array}$ & $\begin{array}{l}\text { Discovery } \\
\text { Reference }\end{array}$ & $\begin{array}{l}\text { Explosion } \\
\text { Epoch }\end{array}$ & $\begin{array}{l}\mathrm{N} \text { of } \\
\text { Spectra }\end{array}$ & Campaign \\
\hline 2009ao & NGC 2939 & 3339 & $\mathrm{Sbc}$ & 0.034 & 54895.0 & CBET 1711 & $54890.7^{\mathrm{a}}(4)$ & 7 & CSP \\
\hline 2009au & ESO 443-21 & 2819 & Scd & 0.081 & 54902.0 & CBET 1719 & $54897.5^{\mathrm{a}}(4)$ & 10 & CSP \\
\hline 2009bu & NGC 7408 & 3494 & $\mathrm{SBc}$ & 0.022 & 54916.2 & CBET 1740 & $54901.9^{c}(8)^{d}$ & 6 & CSP \\
\hline $2009 \mathrm{bz}$ & UGC 9814 & 3231 & $\mathrm{Sdm}$ & 0.035 & 54920.0 & CBET 1748 & $54915.8^{\mathrm{a}}(4)$ & 5 & CSP \\
\hline
\end{tabular}

Notes. Observing campaigns: CTSS = Cerro Tololo Supernova Survey; CT = Calán/Tololo Supernova Program; SOIRS = Supernova Optical and Infrared Survey; CATS $=$ Carnegie Type II Supernova Survey; CSP $=$ Carnegie Supernova Project.

In the first column, the SN name, followed by its host galaxy are listed. In column 3, we list the host galaxy heliocentric recession velocity. These are taken from the NASA Extragalactic Database (NED: http://ned.ipac.caltech.edu/) unless indicated by a superscript (sources in table notes). In columns 4 and 5, we list the host galaxy morphological Hubble types (from NED) and the reddening due to dust in our Galaxy (Schlafly \& Finkbeiner 2011) taken from NED. In columns 6, 7, and 8, we list the discovery date, their reference, and the explosion epochs. The number of spectra and the the observing campaign from which each SN was taken are given in columns 9 and 10, and acronyms are listed in the table notes.

a Explosion epoch estimation from SN nondetection.

${ }^{\mathrm{b}}$ Measured using our own spectra.

c Explosion epoch estimation through spectral matching.

${ }^{\mathrm{d}}$ Cases where explosion epochs have changed between Anderson et al. (2014b) and the current work.

e Taken from the Asiago supernova catalog: http://graspa.oapd.inaf.it/ (Barbon et al. 1999).

${ }^{\mathrm{f}}$ The CATS survey performed the follow-up of SN 210, which was discovered by the SN Factory Wood-Vasey et al. (2004) and was never reported to the International Astronomical Union (IAU) to provide an official designation.

(This table is available in machine-readable form.)

with the most spectra (31), followed by SN 2008bk with 26. On average, we have 7 spectra per SN and a median of 6. There are $87 \mathrm{SNe}$ II for which we have five or more spectra, 32 that have 10 or more, and 6 objects with over 15 spectra (SN 1986L，SN 1993K，SN 2007oc，SN 2008ag, SN 2008bk, and SN 2008if). In the current work, $4 \%$ of our obtained spectra are not used for analysis. 3\% correspond to spectra with low $\mathrm{S} / \mathrm{N}$ that does not allow for useful extraction of our defined parameters, while $1 \%$ are related with peculiarities in the spectra (see Section 5 for more details). Despite this, these spectra are still included in the data release and are noted in Table 6.

\subsection{Data Reduction}

Spectral reduction was achieved in the same manner for all data, using IRAF and employing standard routines, including bias subtraction, flat-fielding correction, one-dimensional (1D) spectral extraction and sky subtraction, wavelength correction, and flux calibration. Telluric corrections have only been applied to data obtained after 2004 October.

In Appendix A (spectral series), we show plots with the spectral series for all $\mathrm{SNe}$ of our sample.

\section{Explosion Epoch Estimations}

Before discussing the properties of our sample, in this section, we outline our methods for estimating explosion epochs. The nondetection of $\mathrm{SNe}$ on prediscovery images with high cadence is the most accurate method for determining the explosion epoch for any given SN. Explosion epochs based on nondetections are set to the midpoint between SN discovery and nondetection. The representative uncertainty on this epoch is then $\left(\mathrm{MJD}_{\text {disc }}-\mathrm{MJD}_{\text {non-det }}\right) / 2$. However, within our sample (and for many other current $\mathrm{SN}$ search campaigns) many $\mathrm{SNe}$ do not have such accurate constraints from this method due to the low cadence of the observations.

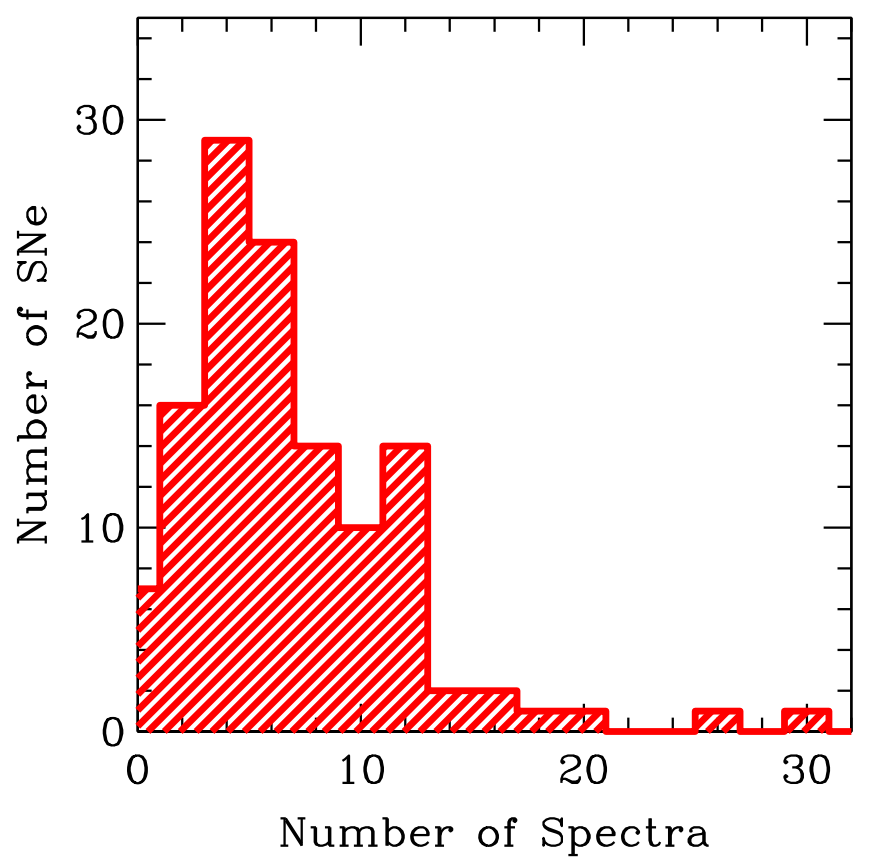

Figure 2. Histogram of the number of spectra per SN. The distribution peaks at four spectra.

Over the last decade, several tools have been published, enabling explosion epoch estimations through matching of observed SN spectra to libraries of spectral templates. Programs such as the Supernova Identification (SNID) code (Blondin \& Tonry 2007), the GEneric cLAssification TOol (Gelato; Harutyunyan et al. 2008), and superfit (Howell et al. 2005) allow the user to estimate the type of supernova and its epoch by providing an observed spectrum. All perform classifications by comparison using different methods. In our analysis, we used only the first two methods: SNID and Gelato. 
We find that Gelato gives a large percentage of their quality of fit to the $\mathrm{H}_{\alpha}$ P-Cygni profile. However, based on our analysis (see Section 8), the most significant changes with time are observed in the blue part of the spectra (i.e., between 4000 and $6000 \AA$ ). Moreover, according to Gutiérrez et al. (2014), the $\mathrm{H}_{\alpha}$ P-Cygni profile shows a wide diversity and there is no clear, consistent evolution with time. In addition, SNID provides the possibility of adding additional templates to improve the accuracy of explosion epoch determinations. We take advantage of this attribute in the following sections by adding new spectral templates, which aid in obtaining more accurate explosion epochs for our sample.

While for many $\mathrm{SNe}$ this spectral matching is required to obtain a reliable explosion epoch, a significant fraction of our sample does have an explosion epoch, constraining SN nondetections before discovery. In cases where the nondetection is $<20$ days before discovery, we use that information to estimate our final values. In cases where this difference is larger than 20 days, we use the spectral matching technique. As a test of our methodology, for nondetection $\mathrm{SNe}$, we also estimate explosion epochs using spectral matching to check the latter's validity (see below for more details).

\subsection{SNID Implementation}

To constrain the explosion epoch for our sample, we compare the first spectrum of each SN II with a library of spectral templates provided by SNID and then, we choose the best match. For each SN, we examined multiple matches, putting emphasis on the fit of the blue part of the spectrum between 4000 and $6000 \AA$. This region contains many spectral lines that display a somewhat consistent evolution with time, unlike the dominant $\mathrm{H}_{\alpha}$ profile at redder wavelengths. Explosion epoch errors from this spectral matching are obtained by taking the standard deviation of several good matches of the observed spectrum of our selected object with those from the SNID library. $\mathrm{H}_{\alpha}$ is the dominant feature in SN II spectra; however, its evolution and morphology varies greatly between $\mathrm{SNe}$ in a manner that does not aid in the spectral matching technique. We therefore ignore this wavelength region.

The red part of the spectrum can be ignored during spectral matching in a variety of ways: (1) using the SNID options; or (2) checking only the match in the blue part. For the former, SNID gives to the user the alternative to modify some parameters. In our case, we can constrain the wavelength range using wmin and wmax. Hence, the structure used is "snid wmin $=3500$ wmax $=6000$ spec.dat". For the latter, we just need to ignore visually the red part of the spectra and explore the matches obtained by SNID until we find a good fit in the blue part. ${ }^{25}$

From the SNID library, we use those template SNe that have well constrained explosion epochs, meaning $\mathrm{SNe}$ II with explosion epoch errors of less than five days (see Table 2). Specifically, we used SN 1999em (Leonard et al. 2002b), SN 1999gi (Leonard et al. 2002a), SN 2004et (Li et al. 2005), SN 2005cs (Pastorello et al. 2006), and SN 2006bp (Dessart et al. 2008). In the database of SNID, there are a total of 166 spectra. However, these templates do not provide a good

\footnotetext{
25 Note that the results obtained from the spectral matching are not altered if you use either all of the visible wavelength spectrum or just the region between 4000 and 6000.
}

Table 2

Reference $\mathrm{SNe}$ II

\begin{tabular}{|c|c|c|c|c|}
\hline SN & $\begin{array}{l}\text { Explosion } \\
\text { Date }\end{array}$ & $\begin{array}{c}\mathrm{V}- \\
\text { maximum } \\
\text { Date }\end{array}$ & $\begin{array}{l}\text { Days from } \\
\text { Explosion to } \\
\text { V-maximum }\end{array}$ & References \\
\hline $1999 \mathrm{em}$ & $2451475.6(5)$ & 2451485.5 & 5 & $\begin{array}{l}\text { Leonard et al. } \\
\text { (2002b) }\end{array}$ \\
\hline 1999gi & $2451518.3(3)$ & 2451530.0 & 12 & $\begin{array}{l}\text { Leonard et al. } \\
\qquad(2002 a)\end{array}$ \\
\hline 2004et & $2453270.5(3)$ & 2453286.6 & 16 & $\begin{array}{c}\text { Li et al. } \\
\text { (2005), Sahu } \\
\text { et al. (2006) }\end{array}$ \\
\hline $2005 \mathrm{cs}$ & 2453547.6 (1) & 2453553.6 & 6 & $\begin{array}{l}\text { Pastorello } \\
\text { et al. (2006) }\end{array}$ \\
\hline $2006 b p$ & 2453833.4 (1) & 2453842.0 & 9 & $\begin{array}{l}\text { Dessart } \\
\text { et al. (2008) }\end{array}$ \\
\hline 1988A & 2447177.2 (2) & $\ldots$ & $\cdots$ & This work \\
\hline $1990 \mathrm{E}$ & 2447935.1 (3) & $\ldots$ & $\cdots$ & This work \\
\hline $1999 \mathrm{br}$ & 2451276.7 (4) & $\ldots$ & $\ldots$ & This work \\
\hline $2003 b n$ & $2452694.5(3)$ & $\ldots$ & $\ldots$ & This work \\
\hline 2003iq & 2452919.5 (2) & $\ldots$ & $\cdots$ & This work \\
\hline $2004 \mathrm{er}$ & 2453271.8 (2) & $\ldots$ & $\cdots$ & This work \\
\hline $2004 \mathrm{fc}$ & $2453293.5(1)$ & $\cdots$ & $\cdots$ & This work \\
\hline $2004 f x$ & $2453303.5(4)$ & $\cdots$ & $\cdots$ & This work \\
\hline $2005 \mathrm{dz}$ & 2453619.5 (4) & $\cdots$ & $\ldots$ & This work \\
\hline $2006 b c$ & 2453815.5 & $\cdots$ & $\cdots$ & This work \\
\hline 2006ee & 2453961.9 (4) & $\cdots$ & $\cdots$ & This work \\
\hline 2006it & 2454006.5 & $\cdots$ & $\cdots$ & This work \\
\hline 2006iw & 2454010.7 (1) & $\cdots$ & $\cdots$ & This work \\
\hline $2006 Y$ & $2453766.5(4)$ & $\ldots$ & $\cdots$ & This work \\
\hline 2007il & $2454349.8(4)$ & $\ldots$ & $\cdots$ & This work \\
\hline 2007 it & $2454348.5(1)$ & $\ldots$ & $\ldots$ & This work \\
\hline $2007 \mathrm{oc}$ & $2454388.5(3)$ & $\ldots$ & $\ldots$ & This work \\
\hline 2008il & $2454825.6(3)$ & $\cdots$ & $\cdots$ & This work \\
\hline 2008in & $2454825.4(2)$ & $\cdots$ & $\cdots$ & This work \\
\hline 2009ао & 2454890.7 (4) & $\ldots$ & $\cdots$ & This work \\
\hline 2009au & 2454897.5 (4) & $\ldots$ & $\ldots$ & This work \\
\hline 2009bz & 2454915.8 (4) & $\cdots$ & $\cdots$ & This work \\
\hline
\end{tabular}

Note. Columns: (1) SN name; (2) Julian date of the explosion epoch; (3) Julian date of the $V$-band maximum; (4) days from Explosion to $V$-band maximum; (5) references.

The first five SNe are included in SNID and are used as templates in this work. Their respective references are presented in column 4. The rest of the $\mathrm{SNe}$ showed after the line are taken from this work as new SNID templates.

coverage of the overall diversity of SNe II within our sample/ the literature. Most of the $\mathrm{SNe}$ in the library are relatively "normal," with only one subluminous event (SN 2005cs). This means that any non-normal event within our sample will probably have poor constraints on its explosion epoch using these templates. For this reason, we decided to use some of our own well-observed SNe II to complement the SNID database.

\subsection{New SNID Templates}

We created a new set of spectral templates using our own SNe II nondetection limits. SNe II are included as new SNID templates if they have errors on explosion epochs (through nondetection constraints) of less than five days. Given this criterion, we included $22 \mathrm{SNe}$, which show significant spectral and photometric diversity. In this manner, the new SNID templates were constructed using $\sim 150$ spectra and prepared using the logwave program included in the SNID packages. 

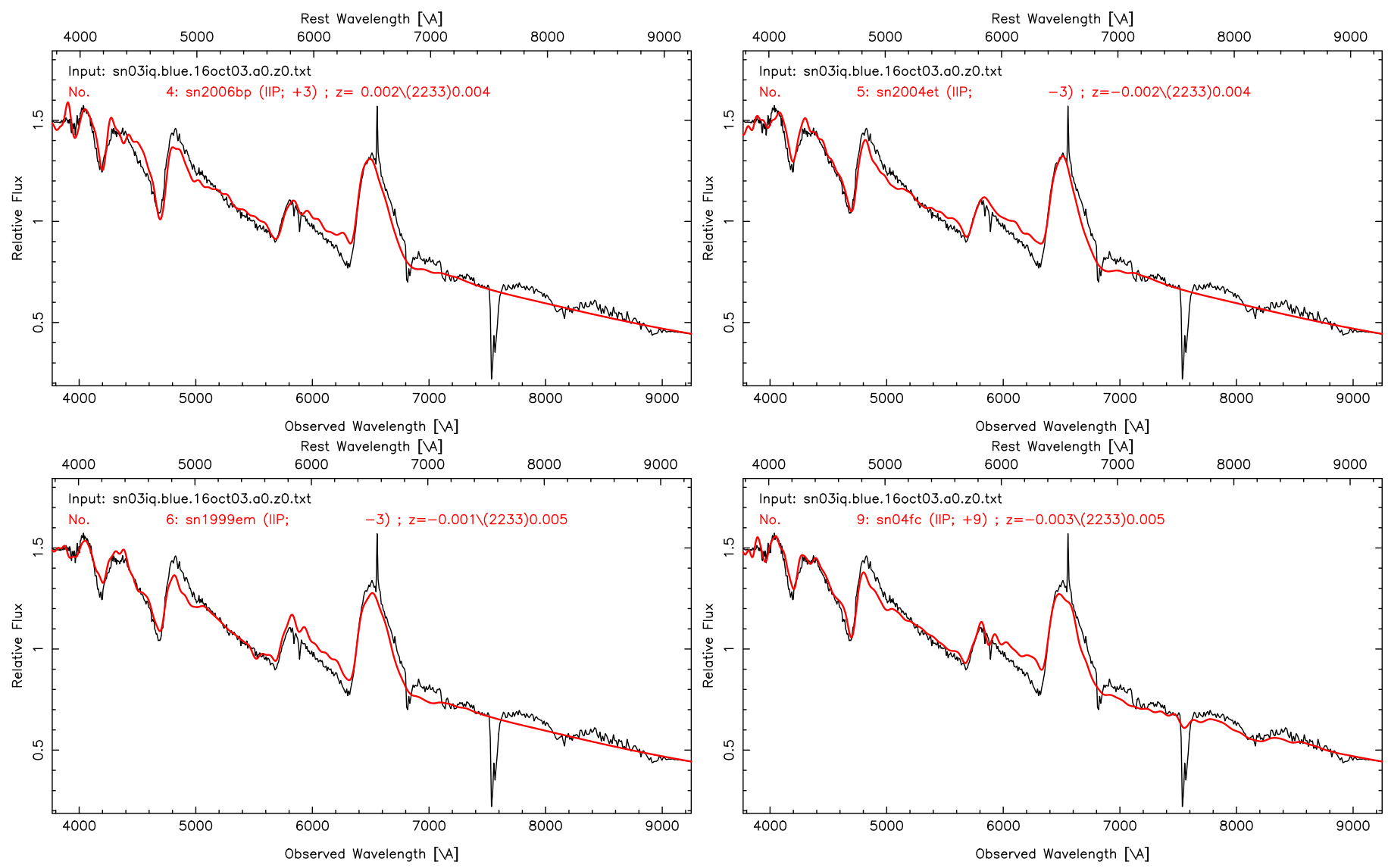

Figure 3. Best spectral matching of SNe 2003iq using SNID. The plots show SN 2003iq compared with SN 2006bp, SN 2004et, SN 1999em, and SN 2004fc at 3, -3 and -3 and 9 days. As the first three SNe are included in the SNID database, they are in respect of the maximum, hence we have to add to them the days between the explosion and the $V$-band maximum (see Table 2) to obtain the explosion epoch. On the other hand, SN 2004fc (included in this work) is in respect of the explosion. Therefore, SN 2003iq has a good match with these SNe at 12,13, 7, and 9 days from explosion, respectively. Taking the average, this means that this spectrum is at $10 \pm 7$ days from explosion.

Adding our own template SNe to the SNID database, we can now use a total of 27 template SNe II to estimate the explosion epoch. Table 2 shows the explosion epoch and the maximum dates in $V$-band for the reference $\mathrm{SNe}$, as well as the explosion epoch for our new templates. We note an important difference between our templates and previous ones in SNID: for the newer templates, epochs are labeled with respect to the explosion epoch, while for the older templates epochs are labeled with respect to maximum light (meaning that one then has to add the "rise time" to obtain the actual explosion date, see Table 2).

\subsection{Explosion Epochs for the Current Sample}

With the inclusion of these 22 SNe to SNID, we estimated the explosion epoch for our full sample. An example of the best match is shown in Figure 3. We can see that the first spectrum of SN 2003iq (October 16th) is best matched with SN 2006bp, SN 2004et, SN 1999em, and SN 2004fc 12, 13, 7, and 9 days from explosion, respectively. Taking the average, we conclude that the spectrum was obtained at $10 \pm 7$ days since the explosion. Table 1 shows the explosion epoch for each $\mathrm{SN}$ as well as the method employed to derive it, while Table 7 shows all the details of spectral matching and nondetection techniques. Appendix B (SNID matches) shows the plots with the best matches for each $\mathrm{SN}$ in our sample.

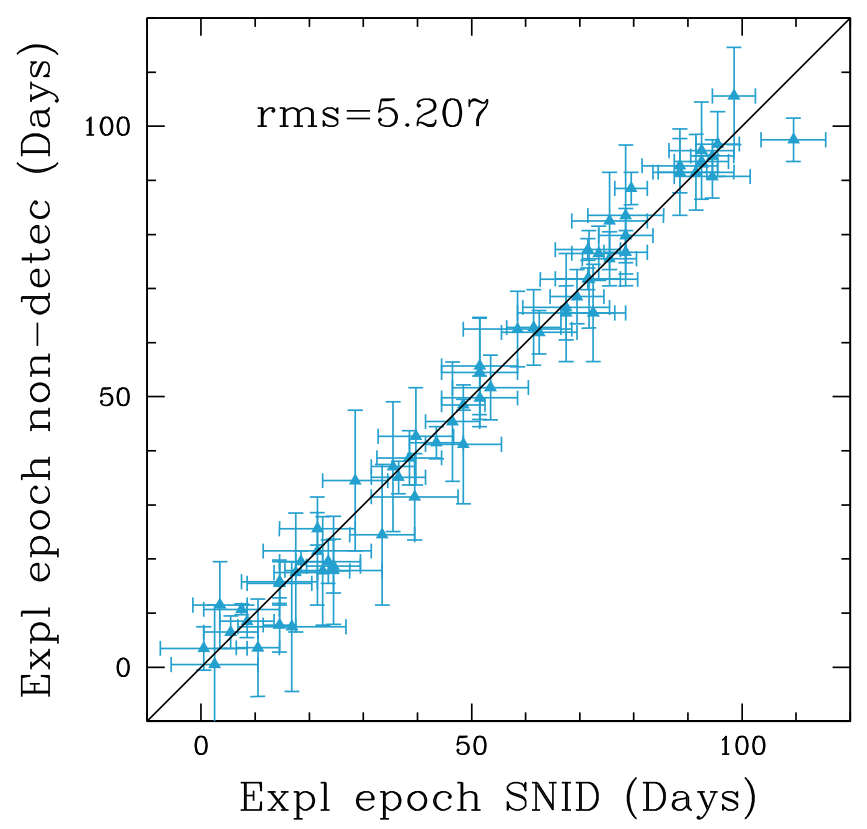

Figure 4. Comparison between spectral matching and nondetection methods.

To check the validity of spectral matching, we compare the explosion epoch estimated with this technique and those with nondetections. These two estimations are displayed in Table 7. 


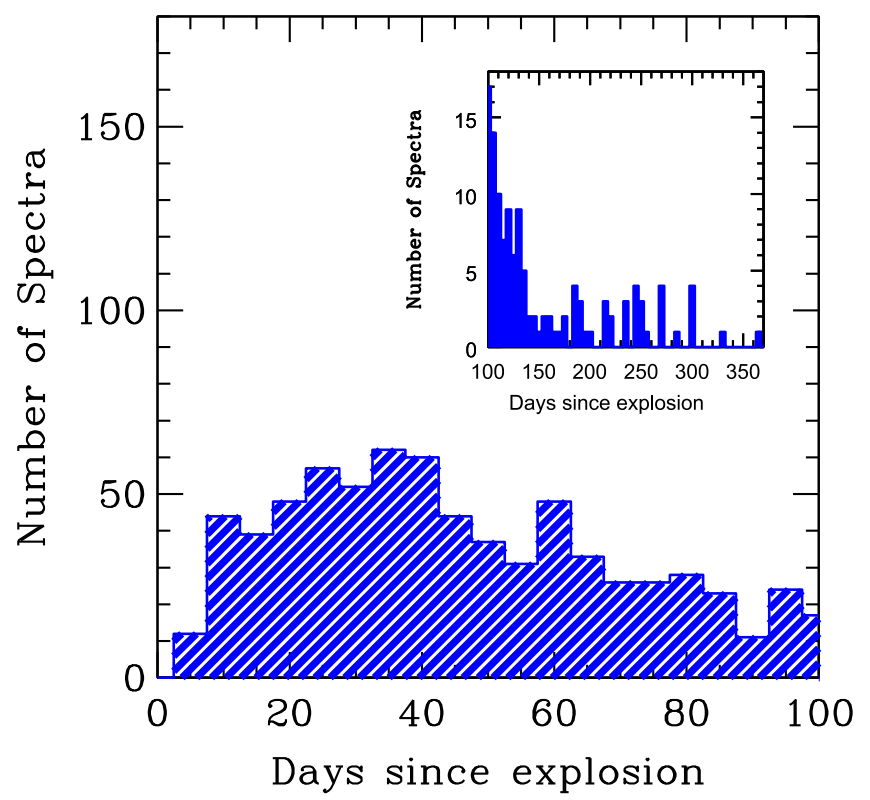

Figure 5. Distribution of the number of spectra as a function of epoch from explosion. The inset on the right shows the same distribution between 100 and 370 days.

From the second to the seventh column, the spectral matching details are shown (spectrum date, best match found, days from maximum-from the SNID templates-days from explosion, average, and explosion date), while from eighth to tenth, those obtained from the nondetection (nondetection date, discovery date, and explosion date). The differences between both methods are presented in the last column. Such an analysis was previously performed by Anderson et al. (2014b), where good agreement was found. With the use of our new templates, we are able to improve the agreement between different explosion epoch constraining methods, thus justifying their inclusion. Figure 4 shows a comparison between both methods, where the mean absolute error between them diminishes from 4.2 (Anderson et al. 2014b) to 3.9 days. Also the mean offset decreases from 1.5 days in Anderson et al. (2014b) to 0.5 days in this work. Cases where explosion epochs have changed between Anderson et al. (2014b) and the current work are noted in Table 1. Nevertheless, although this method works well as a substitute for nondetections, exact constraints for any particular object are affected by any peculiarities inherent to the observed (or indeed template) SN. For example, differences in the color (and therefore temperature) evolution of events can mimic differences in time evolution, while progenitor metallicity differences can delay/hasten the onset of line formation. Further improvements of this technique can only be obtained by the inclusion of additional, well-observed SNe II in the future.

\section{Sample Properties}

As mentioned in Section 2, we have 888 optical spectra of $122 \mathrm{SNe}$ II; however, due to low signal-to-noise $(\mathrm{S} / \mathrm{N})$, we remove 26 spectra of $12 \mathrm{SNe}$ for our analysis. We also remove nine spectra of SN 2005lw because they contain peculiarities that we expect are not intrinsic to the SN (most probably defects resulting from the observing procedure or data reduction). In total, we remove 35 spectra $(\sim 4 \%)$. Figure 5 shows the epoch distribution of our spectra since explosion to
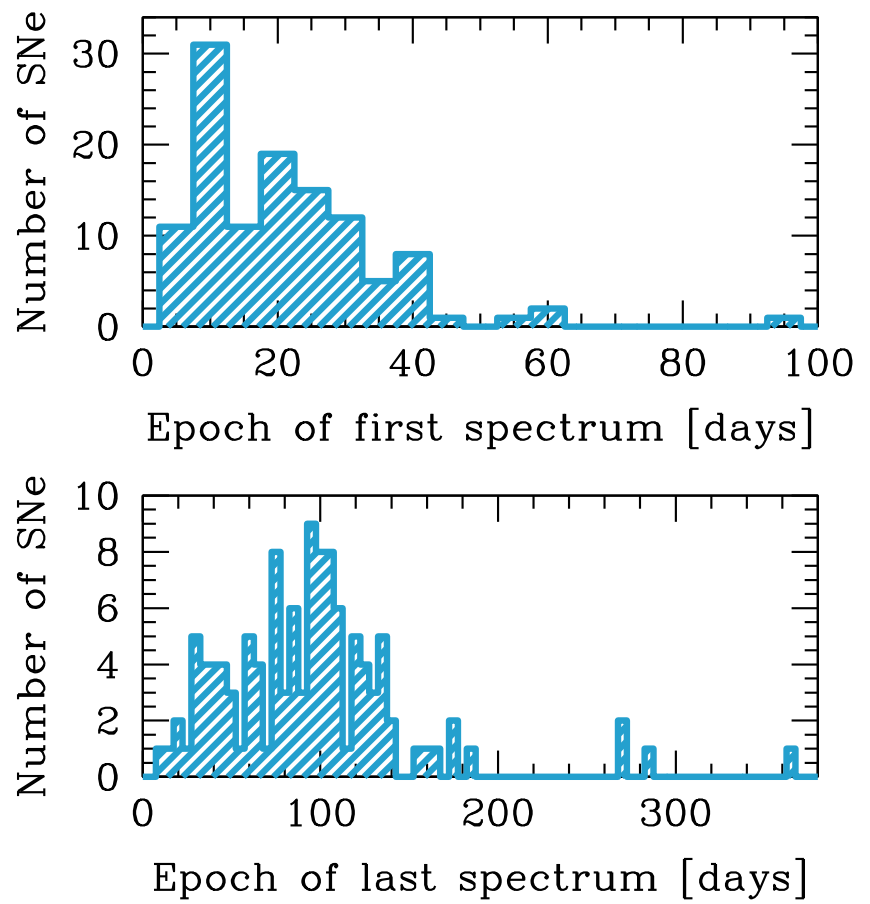

Figure 6. Top: epoch from explosion of first spectrum. Bottom: epoch from explosion of last spectrum.

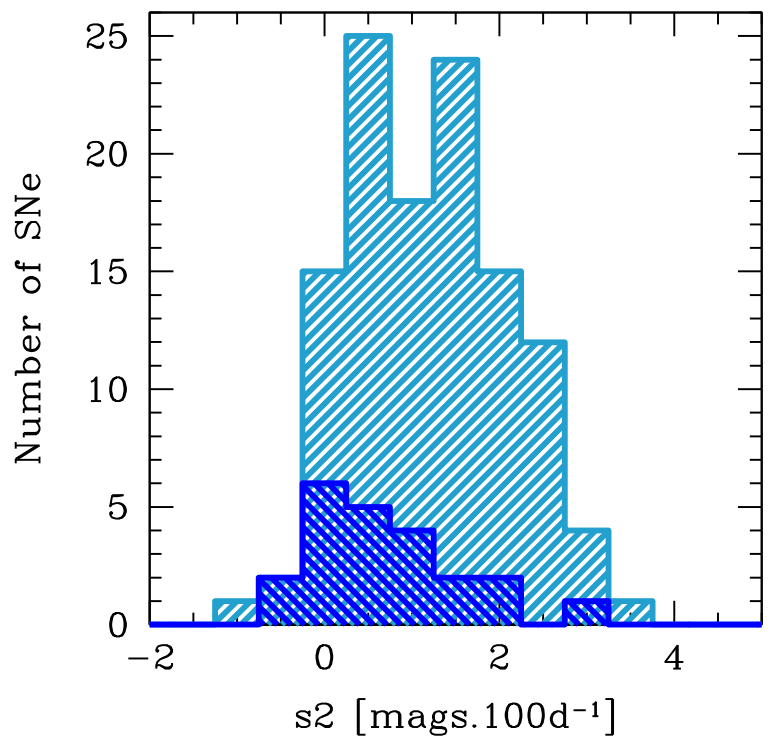

Figure 7. Distribution of the plateau decline $s_{2}$ in $V$ band for $117 \mathrm{SNe}$ of our sample. The blue histogram presents the distribution of " $s_{2}$ " in $V$ band for 22 SNe II used as a new template in SNID.

370 days. One can see the majority $(86 \%)$ of the spectra were observed between 0 and 100 days since explosion, with a total of 738 spectra. Our earliest spectrum corresponds to SN 2008il at $3 \pm 3$ days and SN 2008gr at $3 \pm 6$ days from explosion, while the oldest spectrum is at $363 \pm 9$ days for SN 1993K. $53 \%$ of the spectra were taken prior to 50 days, $3.8 \%$ of which were observed before 10 days for $23 \mathrm{SNe}$. Between $\sim 30$ and 84 days, there are 441 spectra of $114 \mathrm{SNe}$. There are 115 spectra older than 100 days and 27 older than 200 days, corresponding to 45 and $4 \mathrm{SNe}$, respectively. The average of spectra as a function of epoch from explosion is 60 days, while its median is 46 days. 

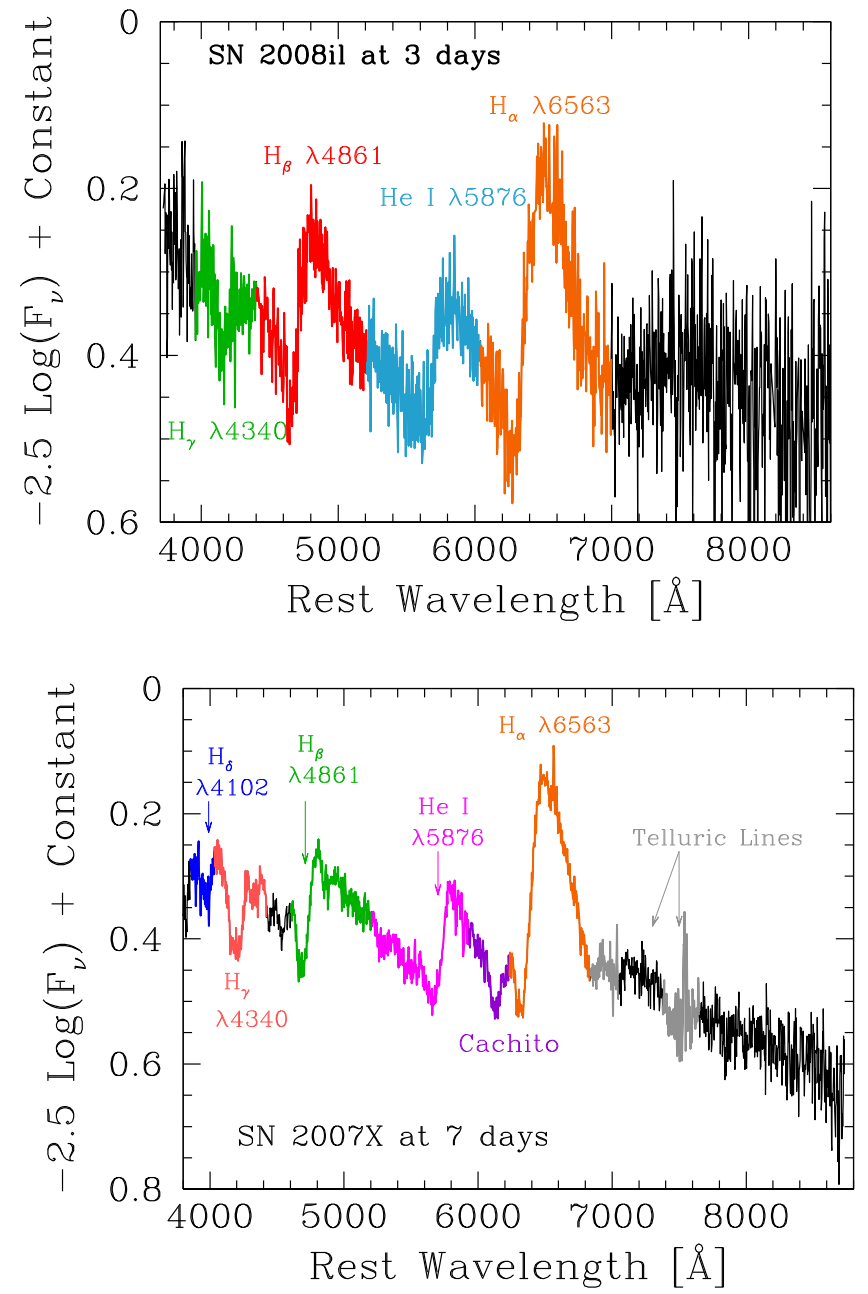

Figure 8. Line identification in the early spectrum of SN 2008 il (top) and SN 2007X (bottom).

Figure 6 shows the epoch distribution of the first and last spectrum for each $\mathrm{SN}$ in our sample. The majority of SNe have their first spectra within 40 days from explosion. There are 31 SNe with their first spectra around 10 days (the peak of the distribution). On the other hand, the peak of the distribution of the last spectrum is around 100 days. Almost all SNe have their last spectra between 30 and 120 days, i.e., in the photospheric phase. There are $11 \mathrm{SNe}$ with their last spectra occuring after 140 days, while only four SNe (SN 1993K, 2003B, SN 2007it, and SN 2008bk) have their last spectra in the nebular phase $(\geqslant 200$ days $)$.

The photometric behavior of our sample in terms of their plateau decline rate ( $s_{2}$; defined in Anderson et al. 2014b) in the $V$ band is shown in Figure 7. For our sample of $117 \mathrm{SNe}$ II, we measure $s_{2}$ values ranging between -0.76 and 3.29 mag 100 day $^{-1}$. Higher $s_{2}$ values mean that the $\mathrm{SN}$ has a faster declining light curve. We can see a continuum in the $s_{2}$ distribution, which shows that the majority of the $\mathrm{SNe}(83)$ have an $s_{2}$ value between 0 and 2 . There are eight objects with $s_{2}$ values smaller than 0 , while three $\mathrm{SNe}$ show a value larger than 3 . The average of $s_{2}$ in our sample is 1.20 . We are unable to estimate the $s_{2}$ value for five $\mathrm{SNe}$, as there is insufficient information from their light curves. The $s_{2}$ distribution for the $22 \mathrm{SNe}$ II used as new templates in SNID is also shown in Figure 7. Although the diversity in the SNID templates increased with the inclusion of these $\mathrm{SNe}$, the template distribution is still biased to low $s_{2}$ values.

\section{Spectral Line Identification}

We identified 20 absorption features within our photospheric spectra, in the observed wavelength range of 3800 to $9500 \AA$. Their identification was performed using the Atomic Spectra Database $^{26}$ and theoretical models (e.g., Dessart \& Hillier $2005,2006,2011)$. Early spectra exhibit lines of $\mathrm{H}_{\alpha} \lambda 6563, \mathrm{H}_{\beta}$ $\lambda 4861, \mathrm{H}_{\gamma} \lambda 4341, \mathrm{H}_{\delta} \lambda 4102$, and $\mathrm{He} \mathrm{I} \lambda 5876$, with the latter disappearing at $\sim 20-25$ days past explosion. An extra absorption component on the blue side of $\mathrm{H}_{\alpha}$ (hereafter "Cachito" "27) is present in many SNe. That line has previously been attributed to the high velocity (HV) features of hydrogen or Si II $\lambda 6533$. Figure 8 shows the main lines in early spectra of $\mathrm{SNe}$ II at 3 and 7 days from explosion. We can see that SN 2008il shows the Balmer lines and He I, while SN 2007X, in addition to these lines, also shows Cachito on the blue side of $\mathrm{H}_{\alpha}$.

In Figure 9, we label the lines present in the spectra of SNe II during the photospheric phase at 31, 70, and 72 days from explosion. Later than $\sim 15$ days, the iron-group lines start to appear and dominate the region between 4000 and $6000 \AA$. We can see Fe-group blends near $\lambda 4554$, and between 5200 and $5450 \AA$ (where we refer to the latter as "Fe II blend" throughout the rest of the text). Strong features such as Fe II $\lambda 4924, \mathrm{Fe}$ II $\lambda 5018, \mathrm{Fe}$ II $\lambda 5169, \mathrm{Sc}$ II $/ \mathrm{Fe}$ II $\lambda 5531$, the $\mathrm{Sc}$ II multiplet $\lambda 5663$ (hereafter "Sc II M"), Ba II $\lambda 6142$, Sc II $\lambda 6247$, O I $\lambda 7774$, O I $\lambda 9263$, and the Ca II triplet $\lambda \lambda 8498,8662$ ( $\lambda 8579$ ) are also present from $\sim 20$ days to the end of the plateau. At 31 days, SN 2003hn shows all of these lines, except Ba II, while at 70 and 72 days, SN 2003bn and SN 2007W show all of the lines. Unlike SN 2003bn, SN 2007W shows Cachito and the "Fe line forest." ${ }^{28}$ The Fe line forest is visible in a small fraction of SNe from 25-30 days (see the analysis in Section 8). As we can see, there are significant differences between two different SNe at almost the same epoch. Later, we analyze and discuss how these differences can be understood in terms of overall diversity of SN II properties.

In the nebular phase, later than 200 days post-explosion, the forbidden lines [Ca II] $\lambda \lambda 7291,7323$, [O I] $\lambda \lambda 6300,6364$, and [Fe II] $\lambda 7155$ emerge in the spectra. At this epoch, $\mathrm{H}_{\alpha}, \mathrm{H}_{\beta}$, Na I $\mathrm{D}$, the Ca II triplet, $\mathrm{O}$ I, and the Fe-group lines between 4800 and $5500 \AA$, and $6000-6500 \AA$ are also still present. Figure 10 shows a nebular spectrum of SN 2007it at 250 days from explosion.

\subsection{The $H_{\alpha}$ P-Cygni Profile}

$\mathrm{H}_{\alpha} \lambda 6563$ is the dominant spectral feature in SNe II. It is usually used to distinguish different $\mathrm{SN}$ types using the initial spectral observation. This line is present from explosion until nebular phases, showing, in the majority of cases, a P-Cygni profile. Although the P-Cygni profile has an absorption and emission component, SNe display a huge diversity in the absorption feature.

\footnotetext{
26 http://physics.nist.gov/asd3

27 Cachito is a Hispanic word that means a small piece of something (like a notch). We use this name to refer to the small absorption components blueward of $\mathrm{H}_{\alpha}$, giving its (until now) previously ambiguous nature.

${ }^{28}$ We label "Fe line forest" to that region around $\mathrm{H}_{\beta}$, where a series of $\mathrm{Fe}$ group (e.g., Fe II $\lambda 4629$, Sc II $\lambda 4670$, and Fe II $\lambda 4924$ ) absorption lines emerge.
} 

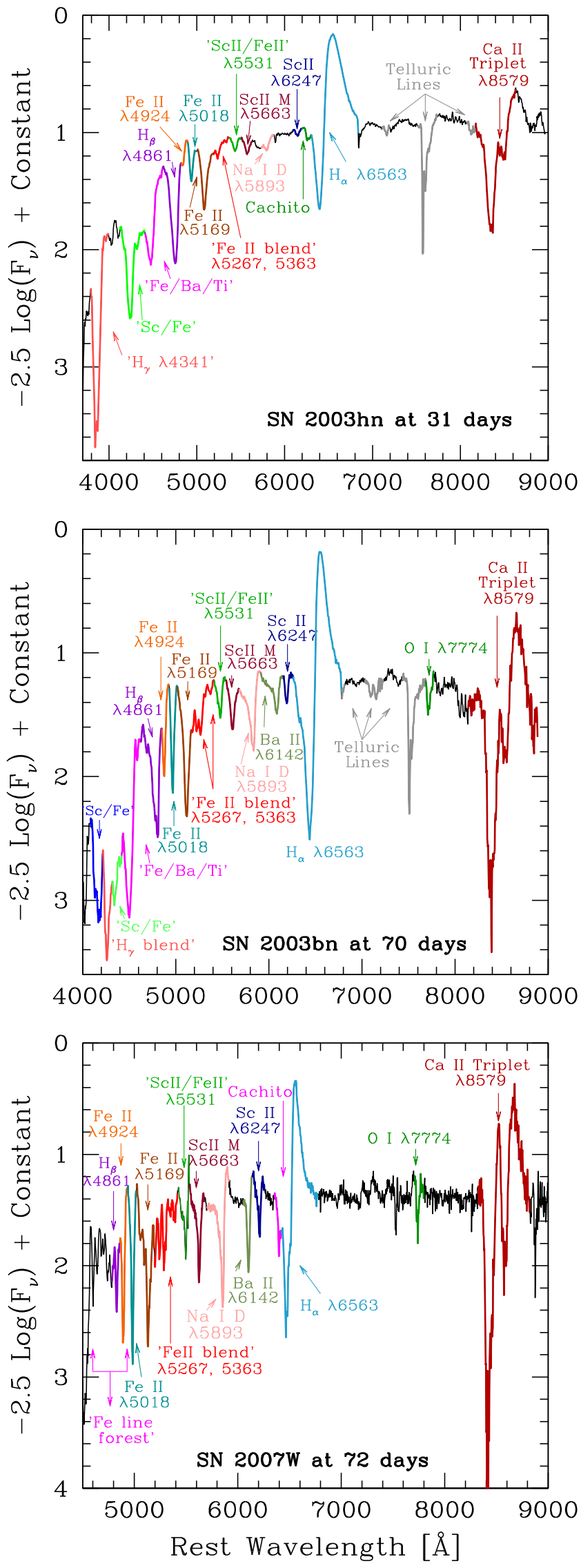

Figure 9. Line identification in the photospheric phase for SNe II 2003hn at 31 days (top), 2003bn at 70 days (middle), and 2007W at 72 days (bottom).

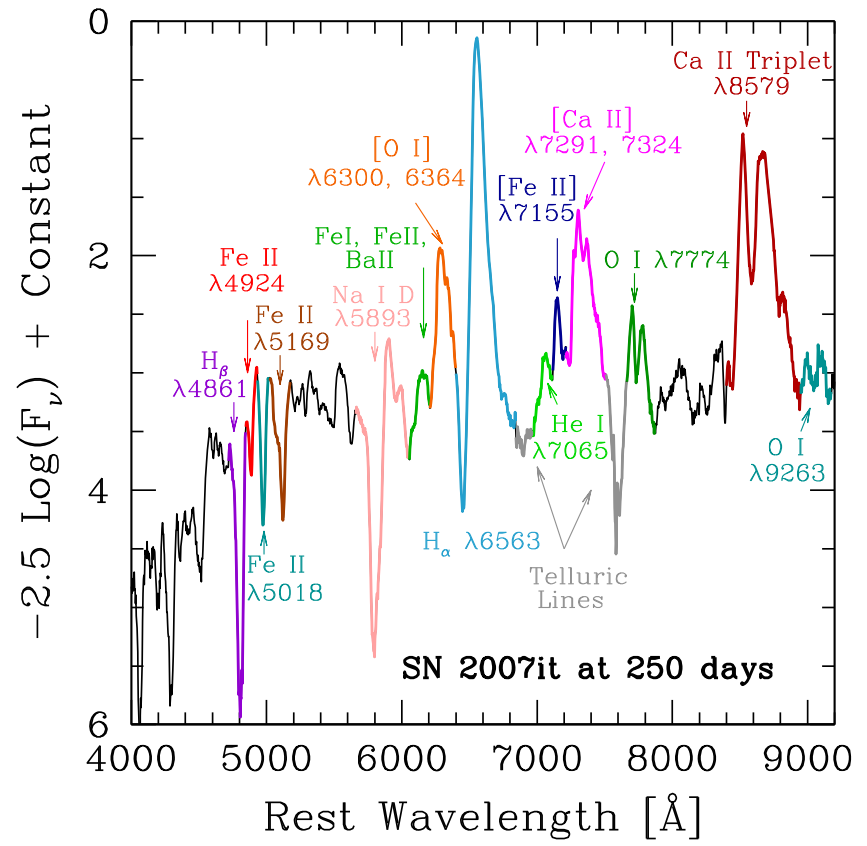

Figure 10. Line identification in the nebular spectrum of SN II 2007it at 250 days from explosion.

Gutiérrez et al. (2014) showed that SNe with little absorption of $\mathrm{H}_{\alpha}$ (smaller absorption to emission $(a / e)$ values) appear to have higher velocities, faster declining light curves, and tend to be more luminous. Here we show that $\mathrm{H}_{\alpha}$ displays a large range of velocities in the photospheric phase, from 9500 to $1500 \mathrm{~km} \mathrm{~s}^{-1}$ at 50 days (see the first two panels in Figure 11, which correspond to the $\mathrm{H}_{\alpha}$ velocity derived from the FWHM of the emission component and from the minimum flux of the absorption, respectively).

The diversity of $\mathrm{H}_{\alpha}$ in the photospheric phase is also observed through the blueshift of the emission peak at early times (Dessart \& Hillier 2008; Anderson et al. 2014a) and the boxy profile (Inserra et al. 2011, 2012). The former is associated with differing density distributions of the ejecta, while the latter with an interaction of the ejecta with a dense CSM. In the nebular phase this shift in $\mathrm{H}_{\alpha}$ emission peak has been interpreted as evidence of dust production in the SN ejecta. Despite the fact that this is an important issue in SNe II, only a few studies (e.g., Sahu et al. 2006; Kotak et al. 2009; Fabbri et al. 2011) have focussed on these features.

In Figure 12, we show an example of the evolution of the $\mathrm{H}_{\alpha}$ P-Cygni profile in SN 1992ba. We can see in early phases a normal profile, which evolves to a complicated profile around 65 days. Cachito on the blue side of $\mathrm{H}_{\alpha}$ is present from 65 to 183 days.

\section{2. $H_{\beta}, \mathrm{H}_{\gamma}$, and $H_{\delta}$ Absorption Features}

$\mathrm{H}_{\beta} \lambda 4861, H_{\gamma} \lambda 4341$, and $\mathrm{H}_{\delta} \lambda 4102$ like $\mathrm{H}_{\alpha}$ are present from the first epochs. In earlier phases, these lines show a P-Cygni profile; however, from $\sim 15$ days the spectra only display the absorption component, giving space to Fe-group lines. The range of velocities of $\mathrm{H}_{\beta}, \mathrm{H}_{\gamma}$, and $\mathrm{H}_{\delta}$ at 50 days post-explosion vary from 8000 to $1000 \mathrm{~km} \mathrm{~s}^{-1}$ (see Figure 11).

Although $\mathrm{H}_{\delta}$ is a common line in SNe II, we do not include a detailed analysis of this line because in many cases the spectra 

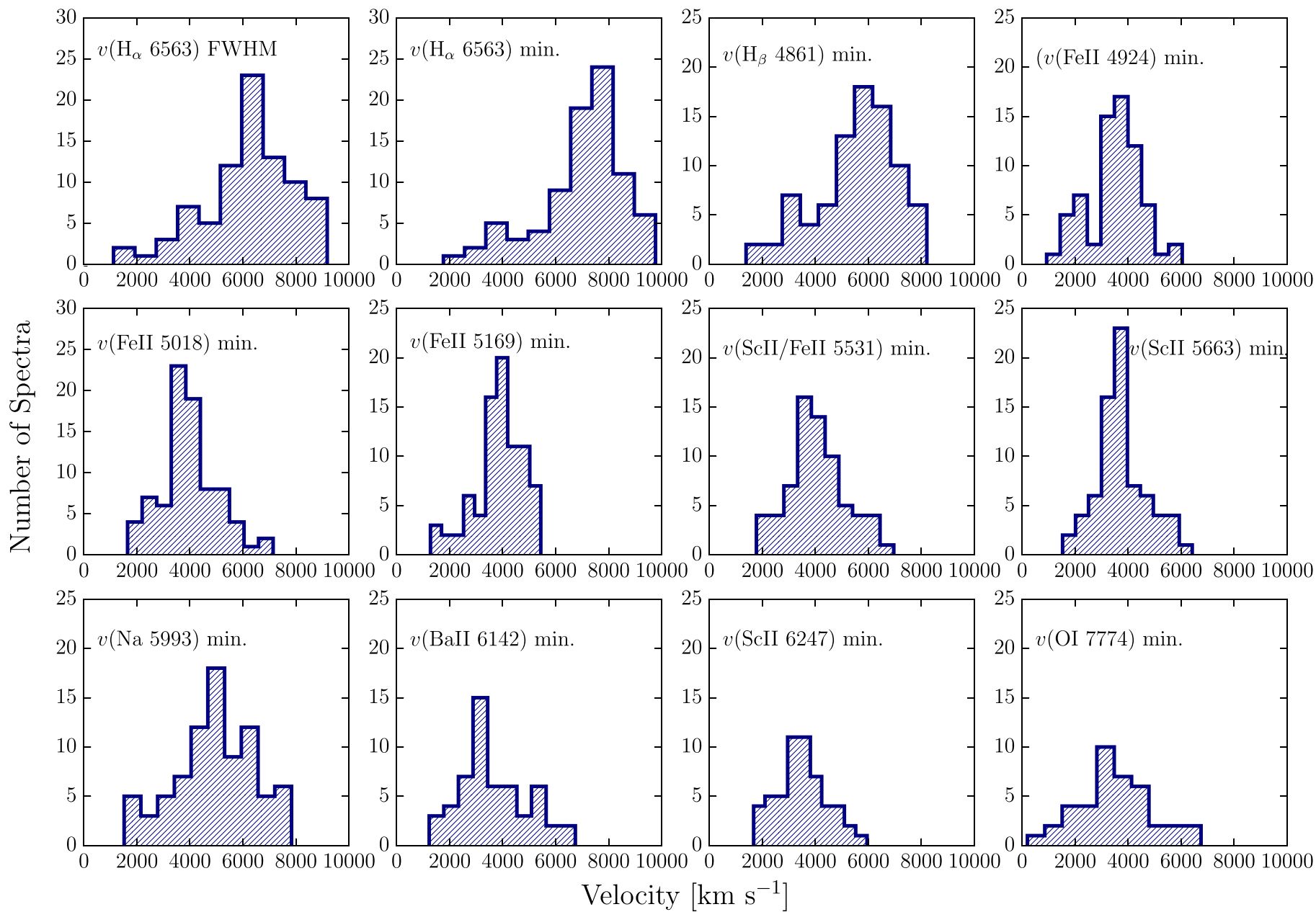

Velocity $\left[\mathrm{km} \mathrm{s}^{-1}\right]$

Figure 11. Distribution of the expansion ejecta velocities for 11 optical features at 50 days. The first two panels show the $\mathrm{H}_{\alpha}$ velocity obtained from the FWHM and from the minimum absorption flux. From the third to the twelfth panel are presented the $\mathrm{H}_{\beta}$, Fe II $\lambda 5018, \mathrm{Fe}$ II $\lambda 4924, \mathrm{Fe}$ II $\lambda 5169$, Sc II/Fe II $\lambda 5531$, Sc II $\lambda 5663$, Na I D $\lambda 5893$, Ba II $\lambda 6142$, Sc II $\lambda 6247$, and O I $\lambda 7774$ velocities obtained from the minimum absorption flux.

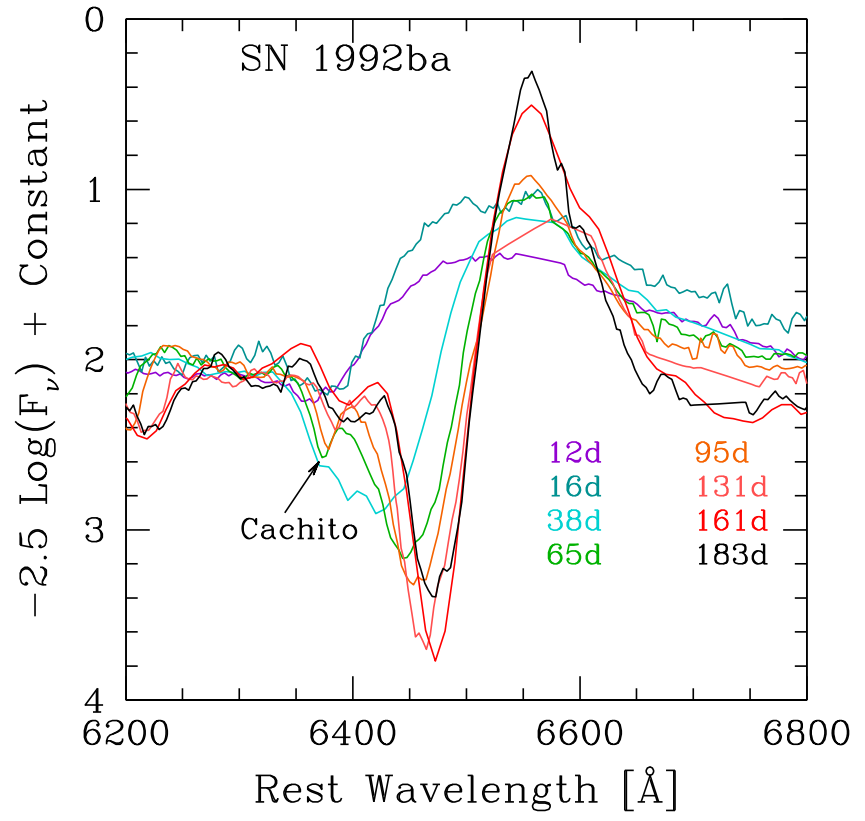

Figure 12. $\mathrm{H}_{\alpha} \mathrm{P}$-Cygni profile evolution in SN 1992ba. The epochs are labeled on the right. are noisy in the blue part of the spectrum. Besides, like other lines in the blue, this line is blended with Fe-group lines later than 30 days.

Around 30 days from explosion $\mathrm{H}_{\gamma}$ starts to blend with other lines, such as Ti II and Fe II. Meanwhile, in a few SNe, the $\mathrm{H}_{\beta}$ absorption feature is surrounded by the Fe line forest. Our later analysis shows that $\mathrm{SNe}$ displaying this behavior are generally dimmer and lower velocity events (see Section 8 for more details).

\subsection{He I $\lambda 5876$ and Na I D $\lambda 5893$}

He I $\lambda 5876$ is present in very early phases when the temperature of the ejecta is high enough to excite the ground state of helium. As the temperature decreases, the $\mathrm{He}$ I line starts to disappear due to low excitation of He I ions (around 15 days; Dessart \& Hillier 2010; Roy et al. 2011). At 30 days, the Na I D $\lambda 5893$ absorption feature arises in the spectrum at a similar position where He I was located. This new line evolves with time to a strong P-Cygni profile, displaying velocities between 8000 and $1500 \mathrm{~km} \mathrm{~s}^{-1}$ at 50 days from explosion (Figure 11).

In many $\mathrm{SNe}$ II (or indeed $\mathrm{SNe}$ of all types), at these wavelengths one often observes narrow absorption features 
arising from slow-moving line-of-sight material from the interstellar medium, ISM (or possibly from circumstellar material, CSM). Such material can constrain the amount of foreground reddening suffered by $\mathrm{SNe}$; however, we do not discuss this here.

\subsection{Fe-group Lines}

When the SN ejecta has cooled sufficiently, Fe II features start to dominate SNe II spectra between 4000 and $6500 \AA$. The first line that appears is Fe II $\lambda 5169$ on top of the emission component of $\mathrm{H}_{\beta}$. With time Fe II $\lambda 5018$ and $\lambda 4924$ emerge between $\mathrm{H}_{\beta}$ and $\mathrm{Fe}$ II $\lambda 5169$. Fe II $\lambda 5169$ becomes a Fe II blend later than $\sim 30-40$ days. At $\sim 50$ days, the 4000-5500 $\AA$ region is completely filled with these lines and the continuum is diminished due to Fe II line-blanketing. The $\mathrm{H}_{\gamma}$ and $\mathrm{H}_{\delta}$ absorption features are blended with Fe-group lines, such as Fe II, Ti II, Sc II, and Sr II. Between 5400 and $6500 \AA$ other metal lines appear in the spectra. Lines such as Sc II/Fe II $\lambda 5531$, Sc II M, Ba II $\lambda 6142$, and Sc II $\lambda 6247$ get stronger with time.

As we can see in Figure 11, the Fe-group lines show a range of velocities between 7000 and $500 \mathrm{~km} \mathrm{~s}^{-1}$ at 50 days. The peak of the distribution of the Fe II group lines velocities is around $4000 \mathrm{~km} \mathrm{~s}^{-1}$. In the case of Ba II, the peak is lower (around $3000 \mathrm{~km} \mathrm{~s}^{-1}$ ).

Although Fe II lines always appear at late phases, few SNe show the iron line forest at 30 days. This feature appears earlier in low velocity/luminosity $\mathrm{SNe}$ (see Section 8).

\subsection{The Ca II NIR Triplet}

The Ca II NIR triplet is a strong feature in the spectra of SNe II. This line appears at $\sim 20-30$ days as an absorption feature, but with time it starts to show an emission component. The Ca II NIR triplet results in a blend of $\lambda 8498$ and $\lambda 8542$ in the bluer part and a distinct component, $\lambda 8662$ on the red part. In SNe II with higher velocities these lines are blended producing a broad absorption and emission profile; however, in low velocity $\mathrm{SNe}$, we see two absorption components and one emission in the red part. The velocities of the Ca II NIR triplet range between 9000 and $1000 \mathrm{~km} \mathrm{~s}^{-1}$ at 50 days. In the nebular phase, the Ca II NIR triplet is also present; however, at this epoch, it only exhibits the emission component.

Although in the majority of our spectra we cannot see Ca II $\mathrm{H}$ and $\mathrm{K} \lambda 3945$, due to the poor signal-to-noise in this region, this line is present in the photospheric phase of SNe II.

While the Ca II NIR triplet is a prominent feature in SNe II, we do not include its analysis in the subsequent discussion, given that the overlap of lines makes a consistent comparison of velocities and pseudo-equivalent widths (pEWs) difficult.

\subsection{O I Lines}

The O I $\lambda \lambda 7772,7775$ doublet (hereafter O I $\lambda 7774)$ and O I $\lambda 9263$ are the oxygen lines in the optical spectra of SNe II. These lines are mainly driven by recombination and they appear when the temperature decreases sufficiently. The O I $\lambda 7774$ line is relatively strong and emerges around 20 days from explosion; however, in the majority of cases it is contaminated by the telluric A-band absorption ( 7600-7630 $)$ ), which hinders detailed analysis. O I $\lambda 9263$ is weaker and appears one month

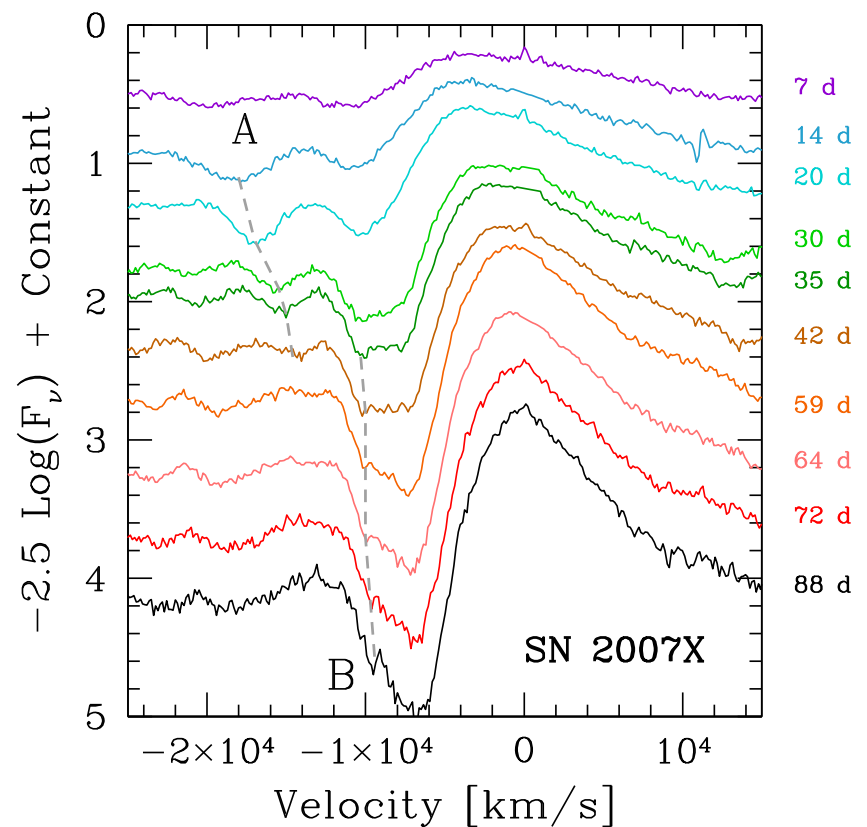

Figure 13. $\mathrm{H}_{\alpha}$ P-Cygni profile of the $\mathrm{SN} 2007 \mathrm{X}$. The epochs are labeled on the right. The dashed lines indicate the velocities for the A and B features, which we call "Cachito."

later than O I $\lambda 7774$. These lines are present until the nebular phase and their expansion velocity at 50 days post-explosion goes from $\sim 7000$ to $500 \mathrm{~km} \mathrm{~s}^{-1}$, as can be seen in Figure 11 .

\subsection{Cachito: Hydrogen High Velocity (HV) Features or the Si II $\lambda 6355$ Line?}

The extra absorption component on the blue side of the $\mathrm{H}_{\alpha}$ P-Cygni profile, called here "Cachito," is seen in early phases in some SNe (e.g., SN 2005cs, Pastorello et al. 2006; SN 1999em, Baron et al. 2000) as well as in the plateau phase (e.g., SN 1999em, Leonard et al. 2002b SN 2007od, Inserra et al. 2011). However, its shape and strength is completely different in the two phases. Baron et al. (2000) assigned the term "complicated P-Cygni profile" to explain the presence of this component on the blue side of the Balmer series. They concluded that these features are due to velocity structures in the expanding ejecta of the SNe II. A few years later, Pooley et al. (2002) and Chugai et al. (2007) argued that this extra component might originate from ejecta-circumstellar (CS) interactions, while Pastorello et al. (2006) earmarked this feature as Si II $\lambda 6355$.

In general, Cachito appears around 5-7 days between 6100 and $6300 \AA$, and disappears at $\sim 35$ days after explosion. Later than 40 days, the Cachito feature emerges closer to $\mathrm{H}_{\alpha}$ (between 6250 and $6450 \AA$ ) and it can be seen until 100-120 days. Figure 13 shows this component in SN 2007X. In early phases, this feature is marked with letter $\mathrm{A}$ and later with letter B. If attributed to $\mathrm{H}_{\alpha}$ the derived velocities are 18,000 and $10,000 \mathrm{~km} \mathrm{~s}^{-1}$, respectively. A detailed analysis of this feature is presented in Section 8.4.

\subsection{Nebular Features}

As mentioned above, $\mathrm{H}_{\alpha}, \mathrm{H}_{\beta}$, the Ca II NIR triplet, $\mathrm{Na}$ ID, $\mathrm{O}$ I, and Fe II are also present in the nebular phase (later than 


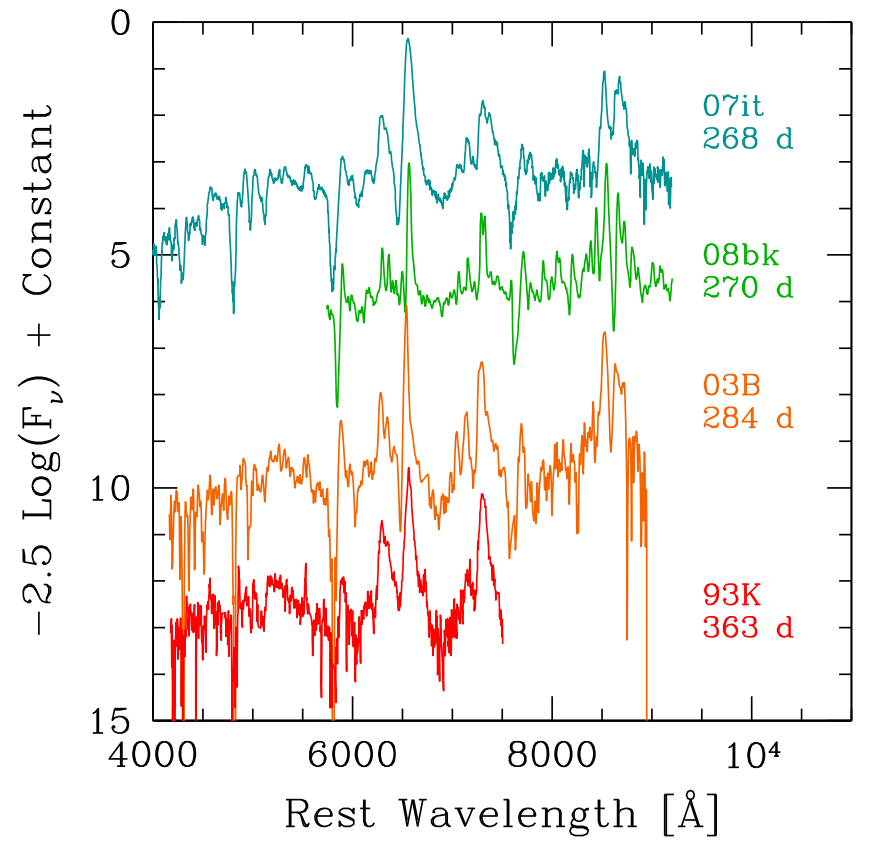

Figure 14. Nebular spectra of seven different $\mathrm{SNe}$ of our sample. The spectra are organized according to epoch.

200 days since explosion); however, in the case of the Ca II NIR triplet, its appearance changes, passing from absorption and emission components to only emission components when the nebular phase starts. The rest of the lines have the same behavior but at much later epochs. The emergence of forbidden emission lines signifies that the spectrum is now forming in regions of low density. At this phase, the ejecta has become transparent, allowing us to peer into the inner layers of the rapidly expanding ejecta. Lines such as [Ca II] $\lambda \lambda 7291,7323$, [O I] $\lambda \lambda 6300,6364$, and $\mathrm{H}_{\alpha}$ are the strongest features visible in the spectra.

The [O I] doublet observed at nebular times is one of the most important diagnostic lines of the helium-core mass (Fransson \& Chevalier 1987; Jerkstrand et al. 2012). Usually the doublet is blended; however, in $\mathrm{SNe}$ with low velocities these lines can be resolved (see, e.g., SN 2008bk). On the other hand, [Fe II] $\lambda 7155$ is easily detectable, but in most cases it is blended with [Ca II] $\lambda \lambda 7291,7323$, and He I $\lambda 7065$, which may hinder their analysis. In Figure 14, we can see the diversity found in the nebular spectra in our sample.

\section{Spectral Measurements}

As discussed previously, SNe II spectra evolve from having a blue continuum with a few lines (Balmer series and $\mathrm{He}$ I) to redder spectra with many lines: Fe II, Ca II, Na I D, Sc II, Ba II, and $\mathrm{O}$ I. To analyze the spectral properties of SNe II, we measure the expansion velocities and pEWs of 11 features in the photospheric phase (see in Table 3 the features used), the ratio of absorption to emission $(a / e)$ of $\mathrm{H}_{\alpha}$ P-Cygni profile before 120 days, and the velocity decline rate of $\mathrm{H}_{\beta}$.
Table 3

Spectral Features Used for the Statistical Analysis in the Photospheric and Nebular Phases

\begin{tabular}{|c|c|c|c|}
\hline $\begin{array}{l}\text { Feature } \\
\text { Name }\end{array}$ & $\begin{array}{l}\text { Rest Wave- } \\
\text { length }^{\text {a }}(\AA)\end{array}$ & $\begin{array}{c}\text { Blueward Limit } \\
\text { Range }^{\mathrm{b}}(\AA)\end{array}$ & $\begin{array}{c}\text { Redward Limit } \\
\text { Range }^{\mathrm{b}}(\AA)\end{array}$ \\
\hline $\mathrm{H}_{\alpha}$ & 6563 & $6000-6300$ & $6900-7100$ \\
\hline $\mathrm{H}_{\beta}$ & 4861 & $4400-4700$ & $4800-4900$ \\
\hline Fe II & 4924 & $4800-4900$ & $4900-4950$ \\
\hline Fe II & 5018 & $4900-4500$ & $5000-5050$ \\
\hline Fe II & 5169 & $5000-5050$ & $5100-5300$ \\
\hline $\mathrm{Na} \mathrm{I}$ & 5893 & $5500-6000$ & $5800-6000$ \\
\hline Sc II & 5531 & $5400-5450$ & $5500-5550$ \\
\hline $\mathrm{Sc}$ II $/ \mathrm{Fe}$ II & 5663 & $5500-5550$ & $5600-5700$ \\
\hline $\mathrm{Ba}$ II & 6142 & $6000-6050$ & $6100-6150$ \\
\hline Sc II & 6247 & $6150-6170$ & $6250-6270$ \\
\hline O I & 7774 & $7630-7650$ & $7750-7780$ \\
\hline
\end{tabular}

Notes.

a The rest wavelengths are weighted averages of the strongest spectral lines that give rise to each absorption feature.

b These limits are necessary in order to account for variations in spectral feature width and expansion velocity among SNe.

\subsection{Expansion Ejecta Velocities}

The expansion velocities of the ejecta are commonly measured from the minimum flux of the absorption component of the P-Cygni line profile. Using the Doppler relativistic equation and the rest wavelength of each line, we can derive the velocity. To obtain the position of the minimum line flux (in wavelength), a Gaussian fitting was employed, which was performed with IRAF, using the splot package. As the absorption component presents a wide diversity (e.g., asymmetries, flat shape, extra absorption components), we repeat the process many times (changing the pseudo-continuum), and the mean of the measurements was taken as the minimum flux wavelength. As our measurement error, we take the standard deviation on the measurements. This error is added in quadrature to errors arising from the spectral resolution of our observations (measured in $\AA$ and converted to kilometers per second) and from peculiar velocities of host galaxies with respect to the Hubble flow $\left(200 \mathrm{~km} \mathrm{~s}^{-1}\right)$. This means that, in addition to the standard deviation error, which realizes the width of the line and $\mathrm{S} / \mathrm{N}$, we take into account the spectral resolution, which, in our case, is the most dominant parameter to determine the error.

The particular case of the $\mathrm{H}_{\alpha}$ velocity was explored in Gutiérrez et al. (2014). Due to the difficulty of measuring the minimum flux in a few $\mathrm{SNe}$ with little or extremely weak absorption components, we derive the expansion velocity of $\mathrm{H}_{\alpha}$ using both the minimum flux of the absorption component and the full width at half maximum (FWHM) of the emission line.

In the case of O I $\lambda 7774$, where the telluric lines can affect our measurement of its absorption minimum, we only use SNe with a clear separation between the two features. This means that the number of SNe with O I measurements is significantly smaller (only $47 \mathrm{SNe}$ ) compared to the other measured features.

\subsection{Velocity Decline Rate}

To calculate the time derivative of the expansion velocity in SNe II, we select the $\mathrm{H}_{\beta}$ absorption line. It is present from the 


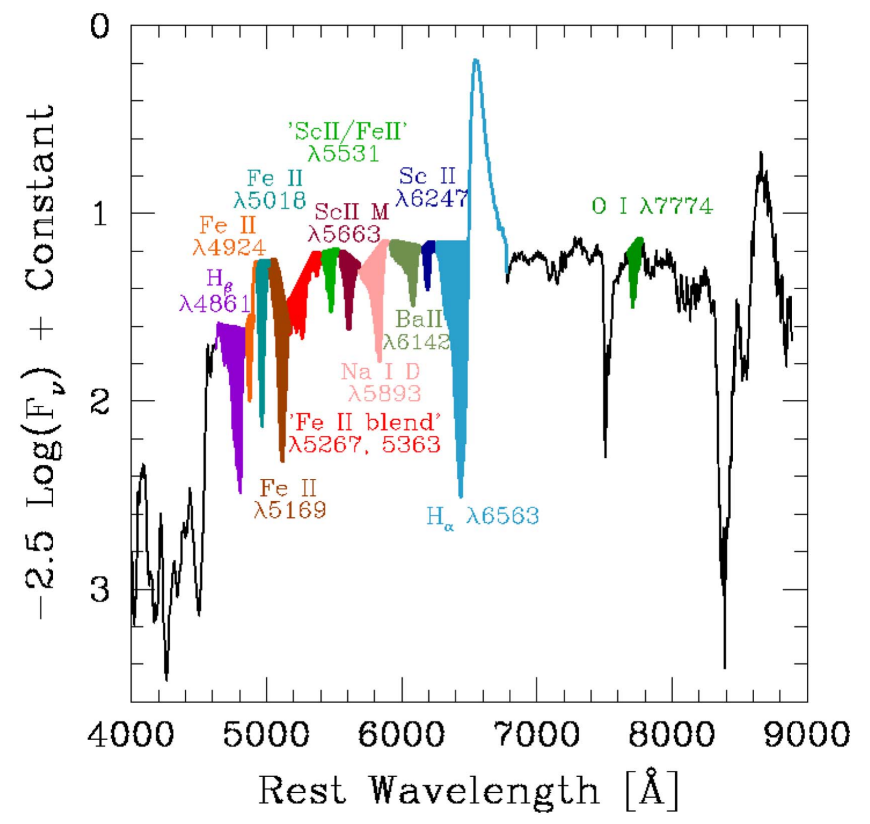

Figure 15. Examples of pEWs used in this work for 11 features in the photospheric phase of SN 2003bn (at 70 days).

early spectra, it is easy to identify and it is relatively isolated. To quantitatively analyze our sample, we introduce the $\Delta v\left(\mathrm{H}_{\beta}\right)$ as the mean velocity decline rate in a fixed phase range $\left[t_{0}, t_{1}\right]$ : $\Delta v\left(\mathrm{H}_{\beta}\right)=\frac{\Delta v_{\mathrm{abs}}}{\Delta t}=\frac{v_{\mathrm{abs}}\left(t_{1}\right)-v_{\mathrm{abs}}\left(t_{0}\right)}{t_{1}-t_{0}}$.

This parameter was measured over the interval $[+15,+30]$ $\mathrm{d},[+15,+50] \mathrm{d},[+30,+50] \mathrm{d},[+30,+80] \mathrm{d}$, and $[+50$, $+80] \mathrm{d}$.

\subsection{Pseudo-equivalent Widths}

To quantify the spectral properties of SNe II, another avenue for investigation is the measurement and characterization of spectral line pEWs. The prefix "pseudo" is used to indicate that the reference continuum level adopted does not represent the true underlying continuum level of the $\mathrm{SN}$, given that in many regions the spectrum is formed from a superposition of many spectral lines. The pEW basically defines the strength of any given line (with respect to the pseudo-continuum) at any given time. The simplest and most often used method is to draw a straight line across the absorption feature to mimic the continuum flux. Figure 15 shows an example of this technique applied to SN 2003bn. We do not include analysis of spectral lines where it is difficult to define the continuum level, due to complicated line morphology, such as significant blending between lines. For example, later than 20-25 days, all absorption features bluer than $\mathrm{H}_{\beta}$ are produced by blends of Fe-group lines plus other strong lines, such as $\mathrm{Ca}$ II $\mathrm{H} \& \mathrm{~K}$ and $\mathrm{H}_{\gamma}$. On the other hand, the Ca II NIR triplet $\lambda \lambda 8498,8662$ shows a profile that depends on the $\mathrm{SN}$ velocity (higher velocity $\mathrm{SNe}$ show a single broad absorption, while low velocity $\mathrm{SNe}$ show two absorption characteristics). These attributes make a consistent analysis between SNe difficult, and therefore we do not include this line in our analysis.

We measure the ratio of absorption to emission $(a / e)$ in $\mathrm{H}_{\alpha}$ until 120 days. In the same way, the pEWs of the absorption lines are measured, we evaluate the pEWs for the emission in $\mathrm{H}_{\alpha}$, thus we have: $a / e=\frac{p \operatorname{EW}\left(H_{\alpha(\mathrm{abs})}\right)}{p \operatorname{EW}\left(H_{\alpha(\mathrm{emis})}\right)}$.

\section{Line Evolution Analysis}

Here we study the time of appearance of different lines within different $\mathrm{SNe}$ and make a comparison of those $\mathrm{SNe}$ with/without specific lines at different epochs. For all lines included in our analysis, we search for their presence in each observed spectrum. Then, at any given epoch, we obtain the percentage of $\mathrm{SNe}$ that display each line. This enables an analysis of the overall line evolution of our sample and whether the speed of this evolution changes between different $\mathrm{SNe}$ of different light curve, spectral, and environment (metallicity) characteristics.

In Figure 16 we show the percentage of $\mathrm{SNe}$ displaying specific spectral features as a function of time. As discussed previously, $\mathrm{H}_{\alpha}$ and $\mathrm{H}_{\beta}$ are permanently present in all the $\mathrm{SNe}$ II spectra from the first days, so we do not include them in the plot. We can see the following.

1. The feature located in the position of $\mathrm{He} / \mathrm{NaI} \mathrm{D}$ is visible in all epochs; however, around 15-25 days, fewer SNe show the line with respect to either the earlier or later spectrum. We suggest that in this epoch the transition from $\mathrm{He}$ I to $\mathrm{Na}$ I $\mathrm{D}$ happens. Therefore, after 30 days, we refer to this line as $\mathrm{NaI} \mathrm{D}$. It is present in $96 \%$ of the spectra from $\sim 35$ days. Later than 43 days, it is present in all spectra.

2. The Ca II NIR triplet is present in $50 \%$ of the sample at $\sim 20$ days. Before 20 days, it is present in $\sim 12 \%$ of the sample, while later than 25 days it is visible in almost all the sample, but with one exception at 38 days. The latter is SN 2009aj, which shows signs of CS interaction in the early phases.

3. $\mathrm{H}_{\gamma}$ blend with Fe-group lines starts at $\sim 20$ days from explosion, growing dramatically at 35-45 days. Only one spectrum at $\sim 46$ days does not show the blend (SN 2008bp).

4. The Fe-group lines start to appear at around 10 days (see Figure 16). The first line that emerges is Fe II $\lambda 5169$. We can see that few $\mathrm{SNe}$ exhibit the absorption feature before 15 days; however, later at 15 days, around $50 \%$ of $\mathrm{SNe}$ show the line and at 30 days all objects have it. The next line that arises is Fe II $\lambda 5018$. This line is seen from 15 days, being present in all SNe later than 40 days. Meanwhile, Fe II $\lambda 4924$ is seen in one spectrum at 13 days (SN 2008br). From 30 days, it is visible in more than $50 \%$ of the spectra. The Sc II/Fe II $\lambda 5531$, Sc II multiplet $\lambda 5668, \mathrm{Ba}$ II $\lambda 6142$, and Sc II $\lambda 6246$ are detectable later than 30 days. The emergence of the Sc II $/ \mathrm{Fe}$ II $\lambda 5531$ and Sc II multiplet $\lambda 5668$ happens at similar epochs, as well as Ba II $\lambda 6142$ and Sc II $\lambda 6246$.

In order to further understand the differences in line-strength evolution of SNe II, we separate the sample into those SNe that do/do not display a certain spectral feature at some specific epoch. We then investigate whether these different samples also display differences in their light curves and spectra. This is done by using the Kolmogorov-Smirnov (KS) test. Presented in Table 4 are all the results obtained with the KS test: $\mathrm{SNe}$ with/without a given line as a function of $a / e$ and $\mathrm{H}_{\alpha}$ velocity at $t_{\text {tran }+10},{ }^{29} M_{\max }, s_{2}$, and metallicity (derived from the ratio of

\footnotetext{
$t_{\text {tran }+10}$ is defined as the transition time (in $V-$ band) between the initial and the plateau decline, plus 10 days. In other words, $t_{\text {tran }}$ marks the start of the recombination phase. (See A14 and Gutiérrez et al. 2014 for more details.)
} 

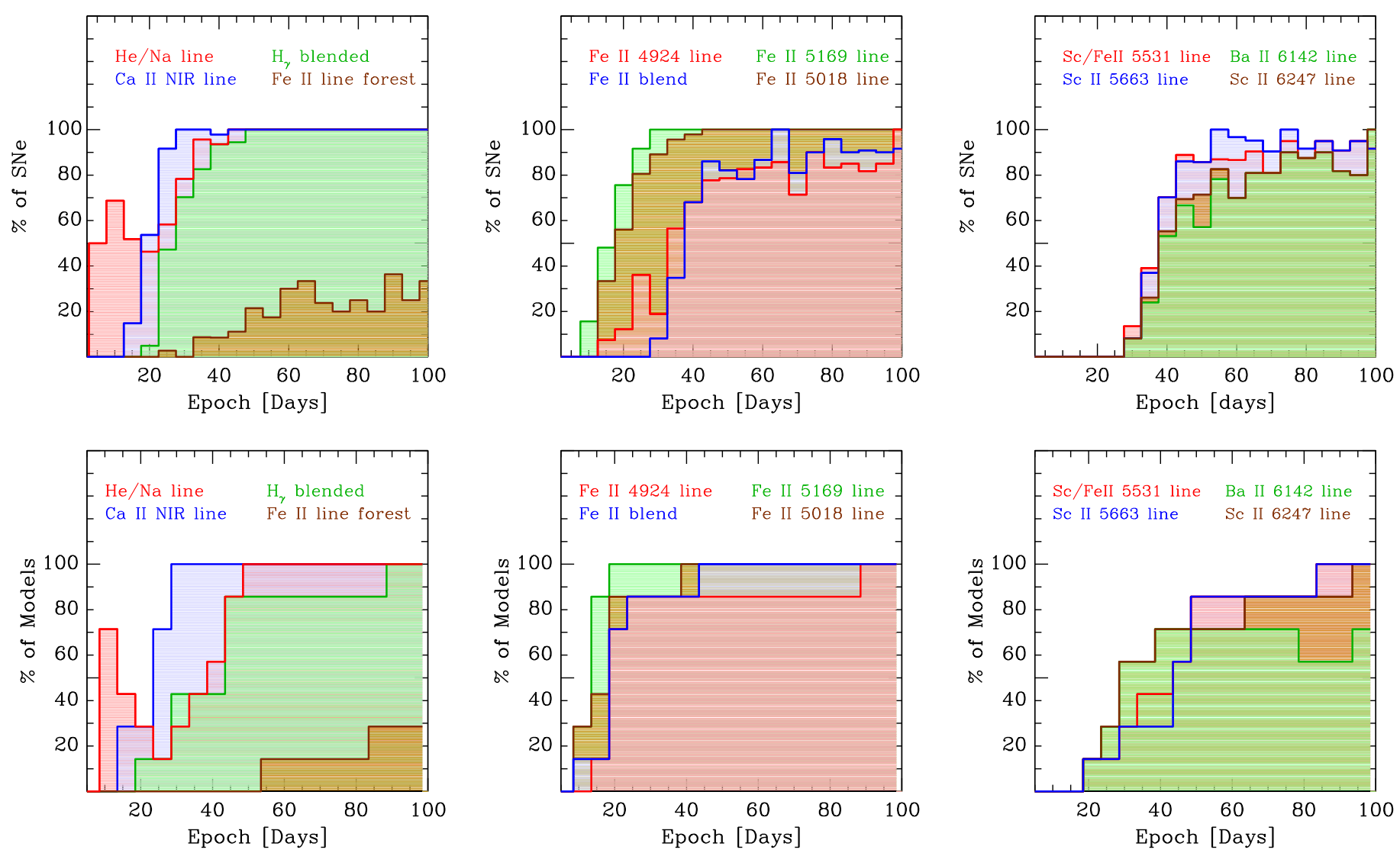

Figure 16. Appearance of different lines in SNe II between explosion and 100 days. Top: from the observed spectra. Bottom: from synthetic spectra (see more details in Table 5).

Table 4

KS-test Values

\begin{tabular}{|c|c|c|c|c|c|c|}
\hline Feature name & $v\left(\mathrm{H}_{\alpha}\right)$ & $a / e$ & $M_{\max }$ & $s_{2}$ & M13 N2 & Epoch (days) \\
\hline Fe II line forest & 5.19 & 9.80 & 2.17 & $3.73 \times 10^{-4}$ & 4.38 & $0-100$ \\
\hline $\mathrm{H}_{\gamma}$ blended & 29.75 & 58.33 & 31.21 & 55.32 & 16.10 & $23-27$ \\
\hline He I 5876 & 18.62 & 1.91 & 30.94 & 25.73 & 46.18 & $18-22$ \\
\hline Ca II IR triplet & 87.73 & 91.97 & 94.47 & 53.30 & 98.82 & $18-22$ \\
\hline Fe II 4924 & 2.82 & 16.84 & 1.09 & 4.80 & 99.40 & $28-32$ \\
\hline Fe II 5018 & 15.68 & 90.80 & 99.02 & 68.84 & 61.53 & $18-22$ \\
\hline Fe II 5169 & 60.76 & 35.15 & 74.88 & 50.83 & 20.30 & $13-17$ \\
\hline Fe II multiplet & 21.38 & 26.75 & 1.00 & 0.25 & 99.28 & $33-37$ \\
\hline Sc II/Fe II 5531 & 75.60 & 89.60 & 30.34 & 45.20 & 1.84 & $38-42$ \\
\hline Sc II multiplet 5663 & 63.54 & 63.54 & 30.34 & 80.10 & 0.79 & $38-42$ \\
\hline Ba II 6142 & 45.75 & 83.58 & 1.90 & 57.43 & 1.29 & $38-42$ \\
\hline Sc II 6247 & 45.76 & 83.58 & 1.89 & 57.42 & 0.52 & $38-42$ \\
\hline
\end{tabular}

Note. Percentage obtained using a KS test to verify if two distributions (with and without each line) are drawn from the same parent population as a function of $v\left(\mathrm{H}_{\alpha}\right)$, $a / e, M_{\max }, s_{2}$, and M13 N2 in an particular epoch. This epoch is shown in the last column of the table. Values lower than $10 \%$ are presented in bold.

$\mathrm{H}_{\alpha}$ to [N II] $\lambda 6583$, henceforth M13 N2 diagnostic; Marino et al. 2013) in a particular epoch. The values of the first four parameters can be found in Table 1 in Gutiérrez et al. (2014), while the metallicity information was obtained from Anderson et al. (2016). We find the following.

1. SNe II that never display the Fe II line forest are distinctly different from those that do display the feature. Specifically, those that do show this feature have slow declining light curves (smaller $s_{2}$ ), are dimmer, and are found to explode in higher metallicity regions within their hosts (see Table 4 for exact statistics).

2. There is less than a $2 \%$ probability that those SNe II, where the He I line is detected between 18 and 22 days post-explosion, arise from the same underlying parent population of $a / e$. This suggests that temperature differences between SNe II affect the morphology of the $\mathrm{H}_{\alpha}$ feature. 

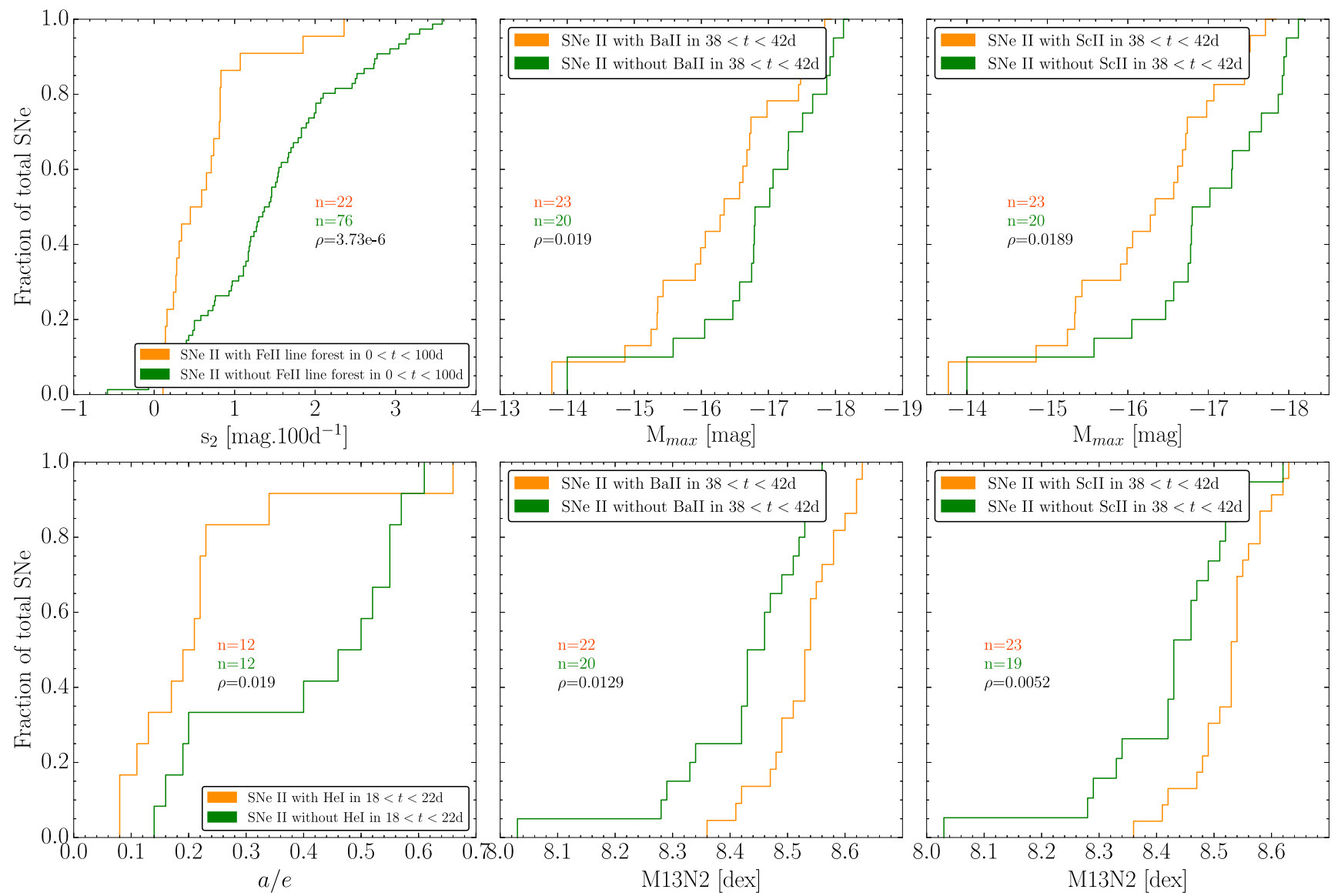

Figure 17. Cumulative distributions of each $\mathrm{SN}$ with/without different lines as a function of spectral and photometric and environment properties. First panel: SNe with/without Fe II line forest between explosion and 100 days as a function of $s_{2}$; second panel: SNe with/without Ba II between 38 and 42 days as a function of $M_{\max }$; third panel: SNe with/without Sc II between 38 and 42 days as a function of $M_{\max }$; fourth panel: SNe with/without He I between 18 and 22 days as a function of $a / e$; fifth panel: SNe with/without Ba II between 38 and 42 days as a function of M13 N2 diagnostic; sixth panel: SNe with/without Sc II between 38 and 42 days as a function of M13 N2 diagnostic.

3. Ba II $\lambda 6142$ and Sc II $\lambda 6247$ are both more likely to be detected at around 40 days post-explosion in dimmer SNe II, with only a $2 \%$ probability that the two populations (with and without these lines) are drawn from the same $M_{\max }$ distribution.

4. Finally, when splitting the SNe II sample into those that do and do not display Sc II/Fe II $\lambda$ 5531, Sc II multiplet $\lambda 5668$, Ba II $\lambda 6142$, and Sc II $\lambda 6247$ at around 40 days post-explosion, we find that there is only around a $1 \%$ probability that the two samples are drawn from the same distribution of metallicity: those $\mathrm{SNe}$ that do not display these lines at this epoch are found to generally explode in regions of lower metallicity within their hosts.

Figure 17 presents the cumulative distributions of the most significative findings obtained with the KS-test analysis.

This analysis was also performed with synthetic spectra for seven different models from Dessart et al. (2013). Four models (m15z2m3, m15z8m3, m15z8m3, and $\mathrm{m} 15 \mathrm{z} 4 \mathrm{~m} 2$ ) show differences in the metallicity, while the rest of the properties are almost the same. The three remaining models have the same metallicity (solar metallicity); however, the other parameters are different: $\mathrm{m} 15 \mathrm{mlt} 1$ has a bigger radius (twice times the radius of the two other models), m15mlt3 has higher kinetic energy, while $\mathrm{m} 12 \mathrm{mlt} 3$ displays a smaller final progenitor mass

Table 5

Model properties

\begin{tabular}{lccrc}
\hline \hline Model & $\begin{array}{c}Z_{\text {final }} \\
\left(M_{\odot}\right)\end{array}$ & $\begin{array}{c}R_{*} \\
\left(R_{\odot}\right)\end{array}$ & $\begin{array}{c}E_{\text {kin }} \\
(B)\end{array}$ \\
\hline m15z2m3 & 0.1 & 14.92 & 524 & 1.35 \\
m15z8m3 & 0.4 & 14.76 & 611 & 1.27 \\
m15z8m3 & 1.0 & 14.09 & 768 & 1.27 \\
m15z4m2 & 2.0 & 12.60 & 804 & 1.24 \\
m15mlt1 & 1.0 & 14.01 & 1107 & 1.24 \\
m15mlt3 & 1.0 & 14.08 & 501 & 1.34 \\
m12mlt3 & 1.0 & 10.50 & 500 & 0.25 \\
\hline
\end{tabular}

Note. Summary of model properties used in this work.

Columns: (1) model name; (2) metallicity; (3) final progenitor mass; (4) progenitor radius; (5) kinetic energy.

and less kinetic energy $\left(1 / 5 E_{\text {kin }}\right.$ compared with the other models). More details are shown in Table 5.

In general, the synthetic spectra show the same behavior (in relation to the appearance of the lines) as observed spectra. However, some differences are found, the majority of which 

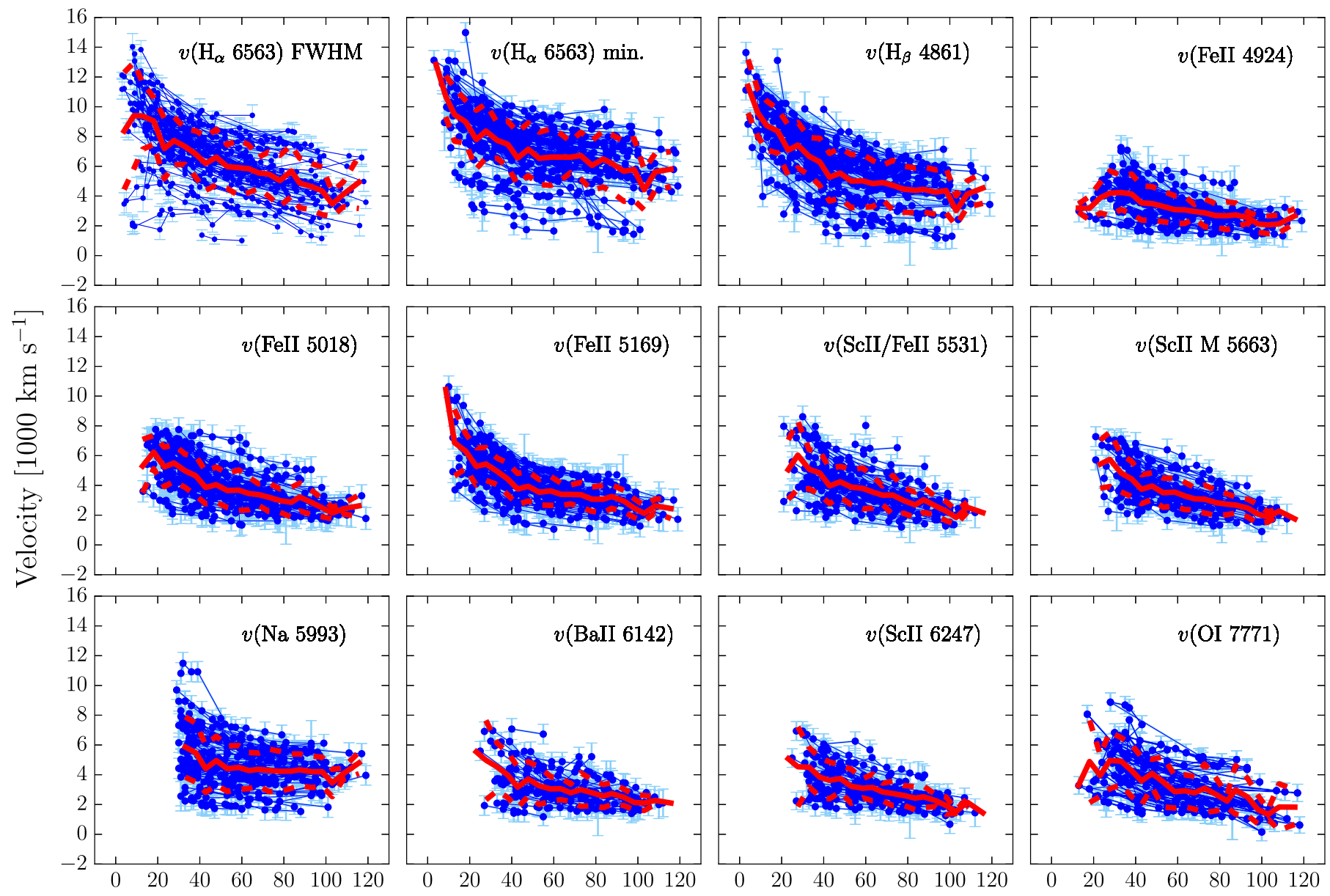

Days from explosion

Figure 18. Expansion velocity evolution for $\mathrm{H}_{\alpha}$ (from the FWHM of emission and the minimum flux absorption), $\mathrm{H}_{\beta}$, Fe II $\lambda 4924$, Fe II $\lambda 5018$, Fe II $\lambda 5169$, Sc II/ Fe II, Sc II multiplet, Na I D, Ba II, Sc II, and O I from explosion to 120 days. The red solid line represents the mean velocity within each time bin, while the dashed red lines indicate the standard deviation. Table 8 presents these values.

are probably related with the low area of parameter space covered by the models that currently exist as compared to the parameter space covered by real events. The transition between He I to Na I D is more evident, and it happens between 18 and 40 days. Although the transition in the models is unambiguously identified by knowing the optical depth of specific lines, in these synthetic spectra, this happens a little bit later than in observed ones. This suggests that the temperature in specific models stays higher for a longer time than the average for observed SNe II. It is also likely that the observed SNe II span a smaller range in progenitor metallicity than the models (that go down to a tenth solar). The $\mathrm{Na} \mathrm{I} \mathrm{D} \mathrm{is} \mathrm{visible} \mathrm{in} 100 \%$ of the sample after 50 days, only 5 days later than the observed spectra. Ca II shows the same behavior in both synthetic and observed spectra; however, $\mathrm{H}_{\gamma}$ is blended in all of the sample later than 90 days, unlike the observed spectra that show it from 45 days. On the other hand, the Fe II line forest is visible from 55 days, in contrast to the observed spectra that show this characteristic from 30 days. This behavior is only present in the spectra of the higher metallicity model (two times solar) and in the lower explosion energy model. The iron lines (Fe II $\lambda 4924$, $\mathrm{Fe}$ II $\lambda 5018, \mathrm{Fe}$ II $\lambda 5169$, and the Fe II blended) are present from $\sim 10$ days. Fe II $\lambda 5169$ is visible in $50 \%$ of the spectra at $\sim 15$

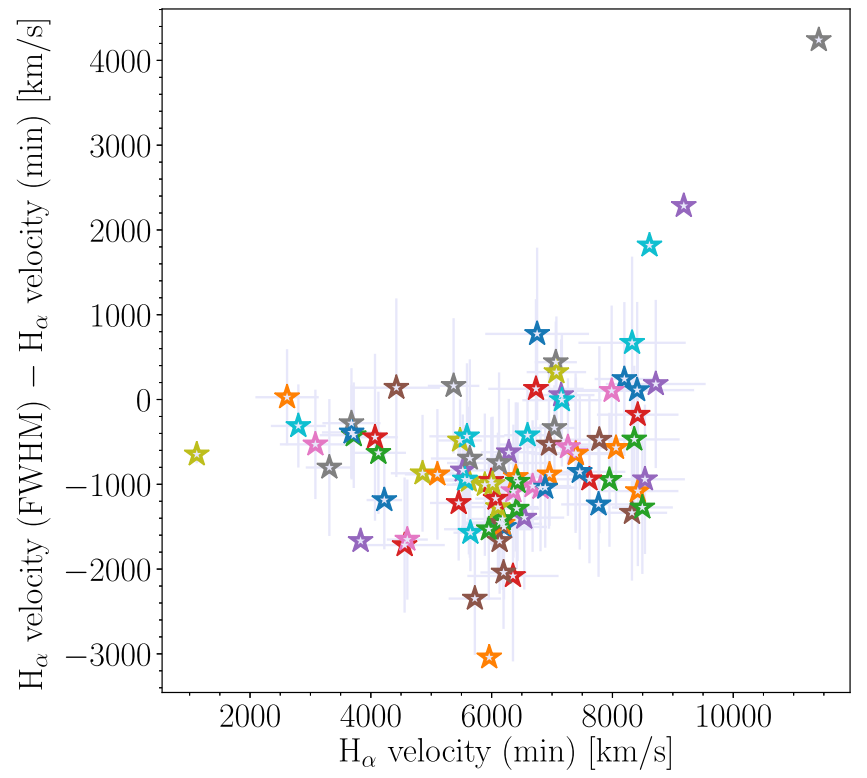

Figure 19. Shifts of the $\mathrm{H}_{\alpha}$ velocity obtained from the FWHM of the emission and from the minimum of the absorption at 50 days post-explosion. 

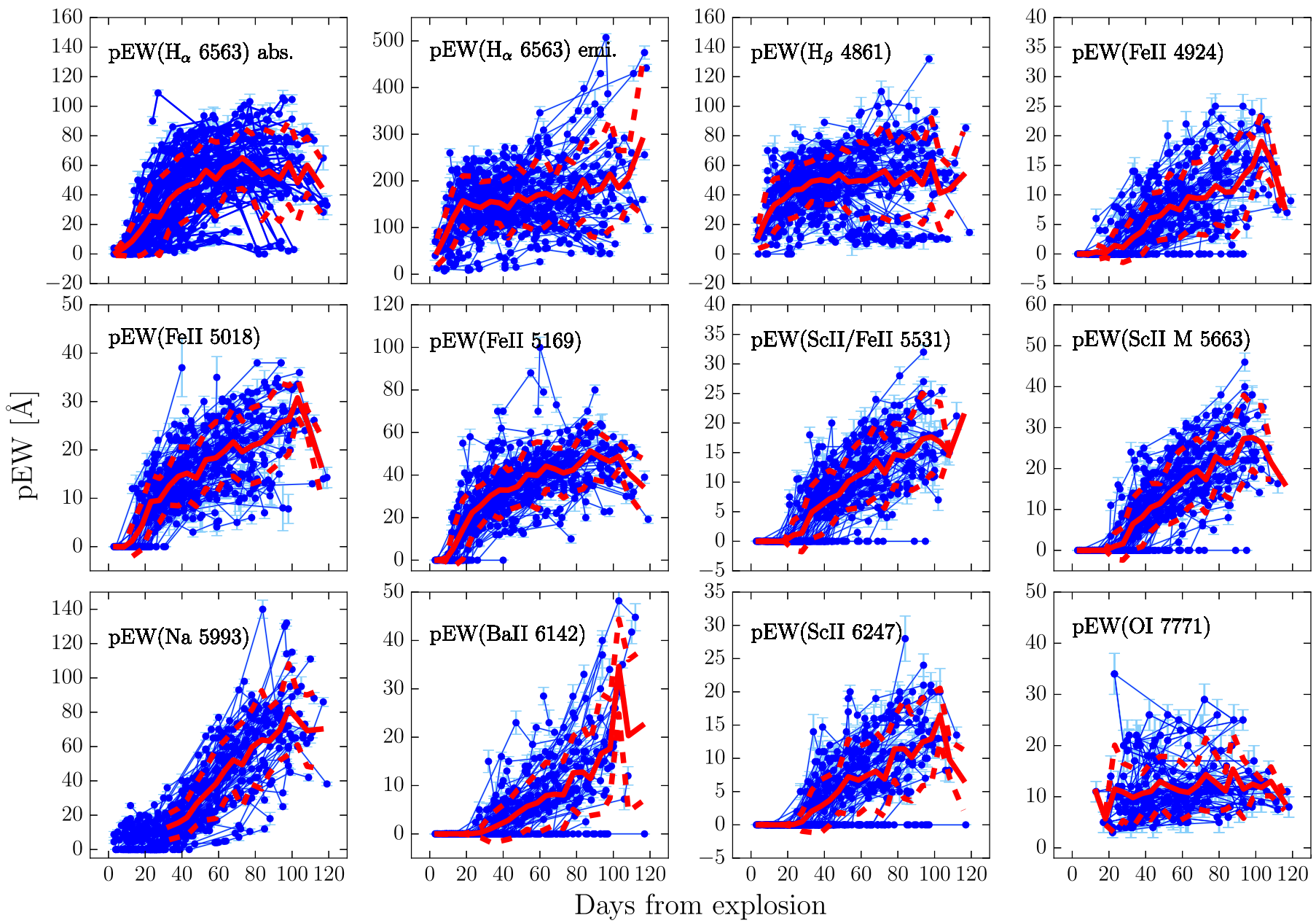

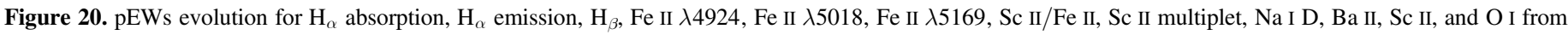

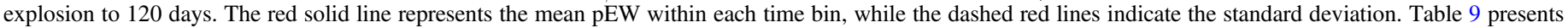
these values.

days, while Fe II $\lambda 4924$ is only visible in $\sim 10 \%$. From 20 days, Fe II $\lambda 5169$ is present in all the synthetic spectra, 10 days earlier than in the observed ones. The behavior of Fe II $\lambda 5018$ is similar in both synthetic and observed spectra, whereas Fe II $\lambda 4924$ starts faster in the models and it is visible in $85 \%$ of the spectra from 30 days. We can see differences in the Fe II blend, which is visible in $100 \%$ of the sample from 50 days in the models; however, in the observed spectra that never happens. More differences are also appreciable between models and observation in $\mathrm{Sc}$ II $/ \mathrm{Fe}$ II $\lambda 5531$, the Sc II multiplet $\lambda 5668$, Ba II $\lambda 6142$, and Sc II $\lambda 6246$. These lines in models arise from 20 days, but in the observations it occurs from 38 to 40 days. Nevertheless, the evolution of the distribution is similar from 50 days. In conclusion, while in general the models produce a time evolution of spectral lines that is quite similar to the observations-supporting the robustness of the models-we observe small differences, suggesting a wider range of explosion and progenitor properties is required to explain the full diversity of observed SNe II.

\subsection{Expansion Velocity Evolution}

Figure 18 shows the velocity evolution of 11 spectral features as a function of time. The first two panels of the plot show the expansion velocity of the $\mathrm{H}_{\alpha}$ feature: on the left, the velocity derived from the FWHM and on the right that derived from the minimum absorption flux. As we can see, the behavior is similar; however, the velocity obtained from the minimum absorption flux is offset between $10 \%$ and $20 \%$ to higher velocities. Figure 19 shows this shift at 50 days. Velocities obtained from the minimum absorption flux are higher around $\sim 1000 \mathrm{~km} \mathrm{~s}^{-1}$. However, it is possible to see few SNe (with higher $\mathrm{H}_{\alpha}$ velocities) showing higher values from the FWHM. Using the Pearson correlation test, we find a weak correlation, with a value of $\rho=0.37$. SNe II with narrower emission components display a larger offset between the velocity from the FWHM and that from the minimum of the absorption. In contrast, those SNe II displaying the highest FWHM velocities present comparatively lower minimum absorption velocities. We note also the presence of two outliers (extreme cases, the lowest and highest value). Figure 11 shows the velocity distribution for the 11 features at 50 days post-explosion. We can see that $\mathrm{H}_{\alpha}$ shows higher velocities than the other lines, followed by $\mathrm{H}_{\beta}$. The lowest velocities are presented by the iron-group lines. In Figure 18, it is possible to see that the $\mathrm{H}_{\beta}$ expansion velocity shows the typical evolution for a homologous expansion and like $\mathrm{H}_{\alpha}$, it is possible to see it from early phases. The iron lines display lower velocities than the Balmer 


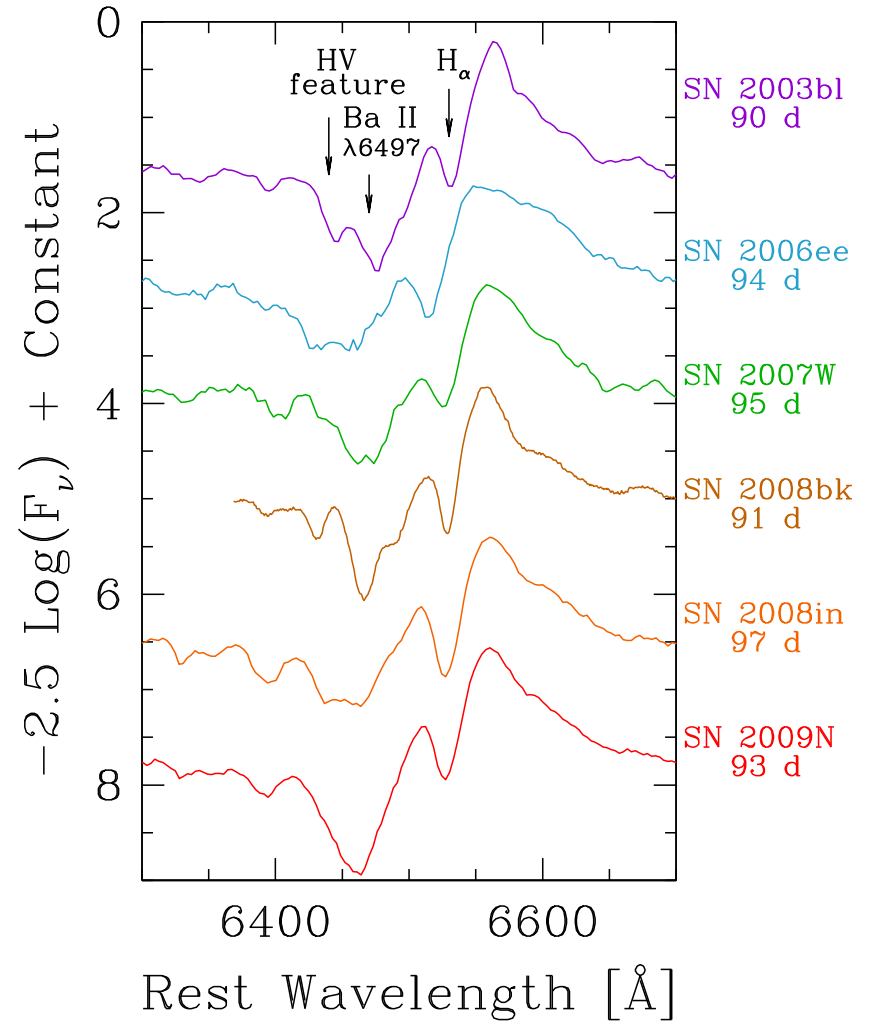

Figure 21. $\mathrm{H}_{\alpha}$ P-Cygni profile of low and intermediate velocity SNe II: 2003bl, 2006ee, 2007W, 2008bk, 2008in, and 2009N around 95 days postexplosion.

lines. So, the highest velocity in $\mathrm{SNe}$ II is found in $\mathrm{H}_{\alpha}$, which implies that it is formed in the outer layers of the SN ejecta. Meanwhile, based on the lower velocities, the iron-group lines form in the inner part, closer to the photosphere. The O I line does not show a strong evolution. As we can see, its velocity evolution is almost flat.

The lowest velocities are found in SN 2008bm, SN 2009aj, and SN 2009au. However, these SNe are distinct from the rest of the population. Unlike subluminous SNe II (such as SN 2008bk and SN 1999br) - that also display low expansion velocities - these events are relatively bright. They also show early signs of CS interactions, e.g., narrow emission lines. By contrast, SN 2007ab, SN 2008if, and SN 2005Z have the largest velocities.

\subsection{Velocity Decline Rate of $H_{3}$ Analysis}

The velocity decline rate of SNe II, denoted as $\Delta v\left(\mathrm{H}_{\beta}\right)$, has not been previously analyzed. We estimate $\Delta v\left(\mathrm{H}_{\beta}\right)$ in five different epochs (outlined above) to understand their behavior. We find that $\mathrm{SNe}$ with a higher decline rate at early times continue to show such behavior at later times. The median velocity decline rate for our sample between 15 and 30 days is $105 \mathrm{~km} \mathrm{~s}^{-1}$ day $^{-1}$, while between 50 and 80 days is $29 \mathrm{~km} \mathrm{~s}^{-1}$ day $^{-1}$. These results show an evident decrease in the velocity decline rate at two different intervals, which is consitent with homologous expansion.

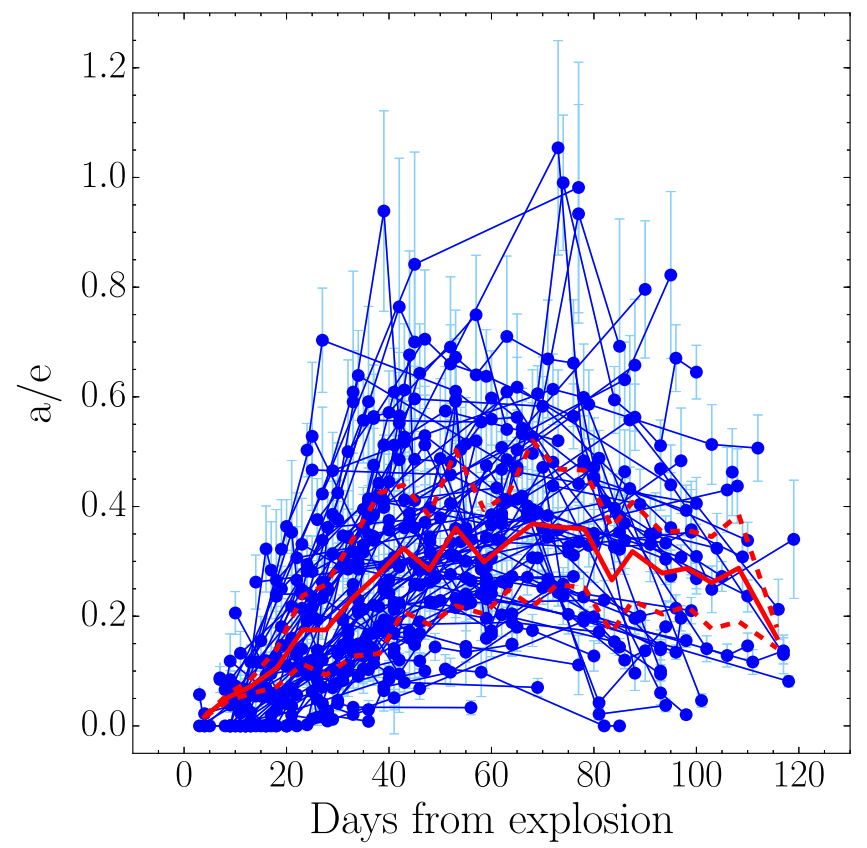

Figure 22. Evolution of the ratio absorption to emission $(a / e)$ of $\mathrm{H}_{\alpha}$ between explosion and 120 days.

\section{3. pEWs Evolution}

The temporal evolution of pEWs for each of the 11 spectral features is shown in Figure 20. In general, the pEWs increase quickly in the first one to two months then level off. The first two panels show the pEW evolution of $\mathrm{H}_{\alpha}$. On the left is displayed the absorption, while on the right the emission component. The absorption component monotonically increases from 0 , increasing to $\sim 100 \AA$; however, in a few SNe its evolution is different: from 70 days, the $\mathrm{pEW}$ decreases significantly. This behavior is observed in low and intermediate velocity SNe (e.g., SN 2003bl, SN 2006ee, SN 2007W, SN 2008bk, SN 2008in, and SN 2009N). Generally, these $\mathrm{SNe}$ show a very narrow $\mathrm{H}_{\alpha} \mathrm{P}$-cygni profile, and at around 70 days from explosion Ba II $\lambda 6497$ appears in the spectra as a dominant feature (see Roy et al. 2011; Lisakov et al. 2017 for more details). In Figure 21, we can see the $\mathrm{H}_{\alpha}$ P-Cygni profile with the presence of Ba II $\lambda 6497$, and the HV feature of hydrogen line (see Section 8.4 for more details) on the blue side of Ba II.

Figure 20 also shows the $\mathrm{H}_{\alpha}$ emission component evolution. An increment in the pEW in the majority of $\mathrm{SNe}$ is appreciable. There are a couple cases (e.g., SN 2006Y), displaying a quasiconstant evolution. The range of $\mathrm{pEW}$ of $\mathrm{H}_{\alpha}$ emission goes up $400 \AA$. In the case of $\mathrm{H}_{\beta}$, we can see that from 60 days there are few $\mathrm{SNe}$ with low pEW values, which show a quasi-constant evolution. SNe with this behavior are those that show the Fe II line forest. The remaining SNe show an increase. The pEWs of iron-group lines grow with time; however, there is a group of $\mathrm{SNe}$ with $\mathrm{pEW}=0$. This indicates that some specific $\mathrm{SNe}$ do not have the line yet. For Sc II/Fe II, the Sc II multiplet, Ba II, and Sc II, this is more obvious. On the other hand, the O I shows a quasi-constant behavior and $\mathrm{NaI} \mathrm{D}$ shows a steady increase. Comparing the values, we can see that the absorption of $\mathrm{H}_{\alpha}, \mathrm{H}_{\beta}$, 


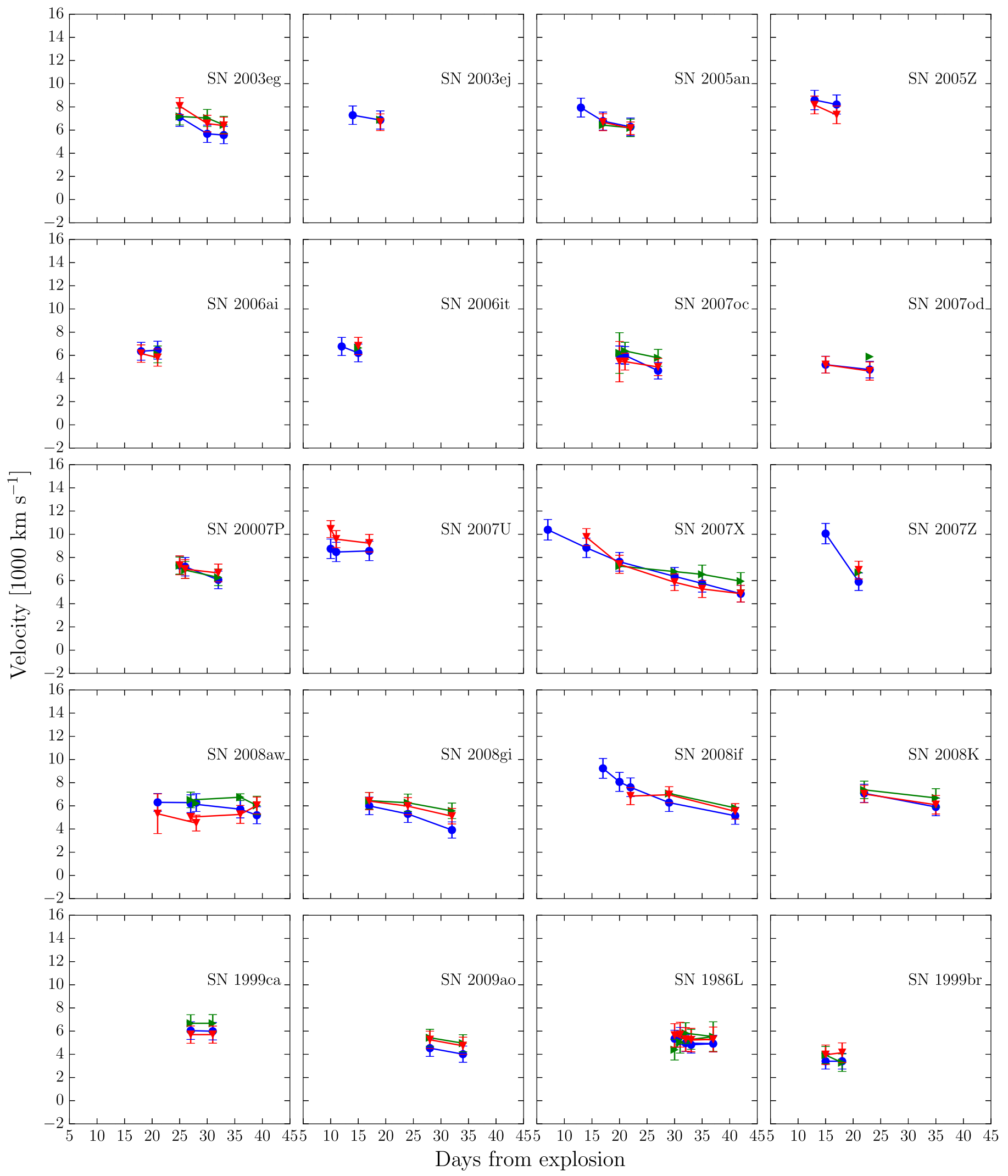

Figure 23. Velocity evolution of Cachito (blue) at early phases compared with Fe II $\lambda 5018$ (green) and Fe II $\lambda 5169$ (red).

and $\mathrm{Na}$ I $\mathrm{D}$ have the highest values (from 0 to $\sim 120$ ), while Fe II $\lambda 4924$, Fe II $\lambda 5018$, Sc II/Fe II, the Sc II multiplet, Ba II, Sc II, and $\mathrm{O}$ I have the lowest ones (from 0 to $\sim 50$ ).
The $a / e$ evolution is displayed in Figure 22. One can see an increase until $\sim 60$ days and then, the quantity remains constant or slightly decreases. 


\subsection{Cachito: Hydrogen HV Features or Si II Line}

The nature of Cachito has recently been studied. Its presence on the blue side of $\mathrm{H}_{\alpha}$ has given rise to multiple interpretations, such as HV features of hydrogen (e.g., Baron et al. 2000; Leonard et al. 2002b; Chugai et al. 2007; Inserra et al. 2011) or Si II $\lambda 6355$ (e.g., Pastorello et al. 2006; Tomasella et al. 2013; Valenti et al. 2014). From our sample, $70 \mathrm{SNe}$ show Cachito in the photospheric phase, between 7 and 120 days post-explosion; however, its behavior, shape, and evolution is different depending on the phase. To investigate the nature of Cachito, we examine the following possibilities.

1. If Cachito is produced by Si II, its velocity should be similar to those presented by other metal lines.

2. If Cachito is related to $\mathrm{HV}$ features of hydrogen, its velocity should be almost the same as those obtained from $\mathrm{H}_{\alpha}$ at early phases. In addition, if it is present, a counterpart should be visible on the blue side of $\mathrm{H}_{\beta}$.

Analyzing our sample, we can detect Cachito in $50 \mathrm{SNe}$ at early phases (before 40 days). Because of the high temperatures at these epochs, the presence of Ba II $\lambda 6497$ is discarded. Assuming that Cachito is produced by Si II, we find that $60 \%$ of SNe present a good match with Fe II $\lambda 5018$ and Fe II $\lambda 5169$ velocities. ${ }^{30}$ Conversely, the rest of the sample shows velocities comparable to those measured at very early phases for $\mathrm{H}_{\alpha}$. Curiously, the Cachito shape is different between the two SN groups. In the former, the line is deeper and broader, while, in the latter, the line is shallow. In Figure 23, we present the velocity comparison for the former group, where a good agreement is found between Cachito, assumed as Si II $\lambda 6355$ (blue), and the iron lines, Fe II $\lambda 5018$ (green) and Fe II $\lambda 5169$ (red).

Later than 40 days, we detect Cachito in $43 \mathrm{SNe}$. Proceeding with the velocity comparison, we can discard its identification as Si II or Ba II $\lambda 6497$ (the latter, visible in few $\mathrm{SNe}$ from 60 days, see Figure 21), which suggests that Cachito is associated to hydrogen. During the plateau, it is possible to see Cachito as a shallow absorption feature only in $\mathrm{H}_{\alpha}$ and/or as a narrow and deeper absorption on the blue side of both $\mathrm{H}_{\alpha}$ and $\mathrm{H}_{\beta}$ (see an example in Figure 24). According to Chugai et al. (2007), the interaction between the SN ejecta and the RSG wind should result in the emergence of these HV absorption features. They argue that the existence of a shallow absorption feature is the result of the enhanced excitation of the outer unshocked ejecta, which is visible on the blue side of $\mathrm{H}_{\alpha}$ (and He I 10830). At early times, the $\mathrm{H}_{\beta}$ Cachito feature is not predicted by Chugai et al. (2007), who argue that the optical depth is too low at the line-forming region. They also discuss that in addition to the $\mathrm{HV}$ shallow absorption, an $\mathrm{HV}$ notch is formed in the cool dense shell (CDS) located behind the reverse shock. Given the relatively high $\mathrm{H}_{\alpha}$ optical depth of the CDS, a counterpart could be seen in $\mathrm{H}_{\beta}$ as well. We found that $63 \%$ of the $\mathrm{SNe}$ with Cachito during the plateau show a counterpart in $\mathrm{H}_{\beta}$ with the same velocity as that presented on $\mathrm{H}_{\alpha}$, which favors the interpretation as CS interaction. The $\mathrm{HV}$ notch of $\mathrm{HI}$ is found in $27 \mathrm{SNe}$; however, in the low velocity/luminosity $\mathrm{SNe}$, it is only present in $\mathrm{H}_{\alpha}$. After 50 days, the blue part of the spectrum $(<5000 \AA)$ is

\footnotetext{
${ }^{30}$ Four SNe show a good match with Si II in very early phases, but between 30 and 40 days they do not show it. They also show a different shape.
}

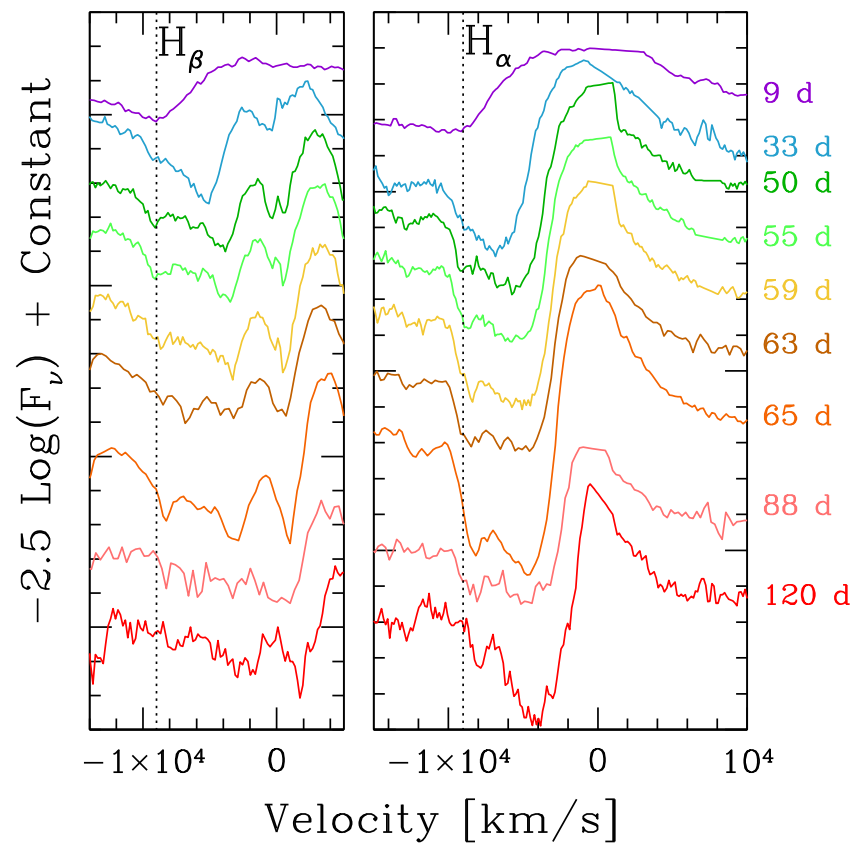

Figure 24. Spectral evolution of $\mathrm{H}_{\alpha}$ and $\mathrm{H}_{\beta}$ lines of SN 2004fc. The dotted lines correspond to the $\mathrm{HV}$ features seen on the blue side of $\mathrm{H}_{\alpha}$ and $\mathrm{H}_{\beta}$ from 50 to 120 days. We can see that the HV features show a velocity evolution from $\sim 9000$ to $\sim 8000 \mathrm{~km} \mathrm{~s}^{-1}$.

dominated by metal lines, which may hinder its detection. Nonetheless, we argue that these can be HV H I because at least one low velocity/luminosity SN, SN 2006ee, shows a Cachito feature on the blue side of both $\mathrm{H}_{\alpha}$ and $\mathrm{H}_{\beta}$, at around 50 days with consistent velocities. A summary of the analysis is displayed in Figure 25, where the $\mathrm{H}_{\alpha}$ (red), $\mathrm{HV} \mathrm{H}_{\alpha}$ (blue), $\mathrm{H}_{\beta}$ (cyan), and $\mathrm{HV} \mathrm{H}_{\beta}$ (green) velocity evolution is presented for $20 \mathrm{SNe}$.

In addition to the $70 \mathrm{SNe}$ where Cachito can be identified either with $\mathrm{Si}$ II or HV features of $\mathrm{H}$ I, we find six SNe II that display Cachito at certain epochs; however, its exact properties do not align with the above interpretations (because of differences in shape and/or velocity). These are SN 2003bl, SN 2005an, SN 2007U, SN 2008br, SN 2002gd, and SN 2004fb. In summary, $59 \%$ of the full SNe sample show Cachito at some epoch, while $41 \%$ never show this feature. Soon after shock break-out, all SNe II have extremely high temperature ejecta. Therefore, if we were able to obtain spectral sequences shortly after explosion, the Si II feature would always be observed. However, observationally, this is not the case because there are many SNe II within our sample without Si II detections. This is simply an observational bias, due to the lack of data at very early times. Nevertheless, for SNe II that stay hotter for longer, the probability of detecting Si II becomes larger. We therefore speculate that $\mathrm{SNe}$ II that have detected $\mathrm{Si}$ II at early times have larger radii, which leads to a slower cooling of the ejecta and hence facilitates Si II detection. Interestingly, when we split the sample into those SNe II that do and do not display the Si II line, those where the line is detected are found to have lower $a / e$ values, with only a $4 \%$ chance that the two populations are drawn from the same underlying distribution. This is also consistent with the previous finding that those $\mathrm{SNe}$ II with evident $\mathrm{He}$ I detections at around 20 days post-explosion are also found to have lower 


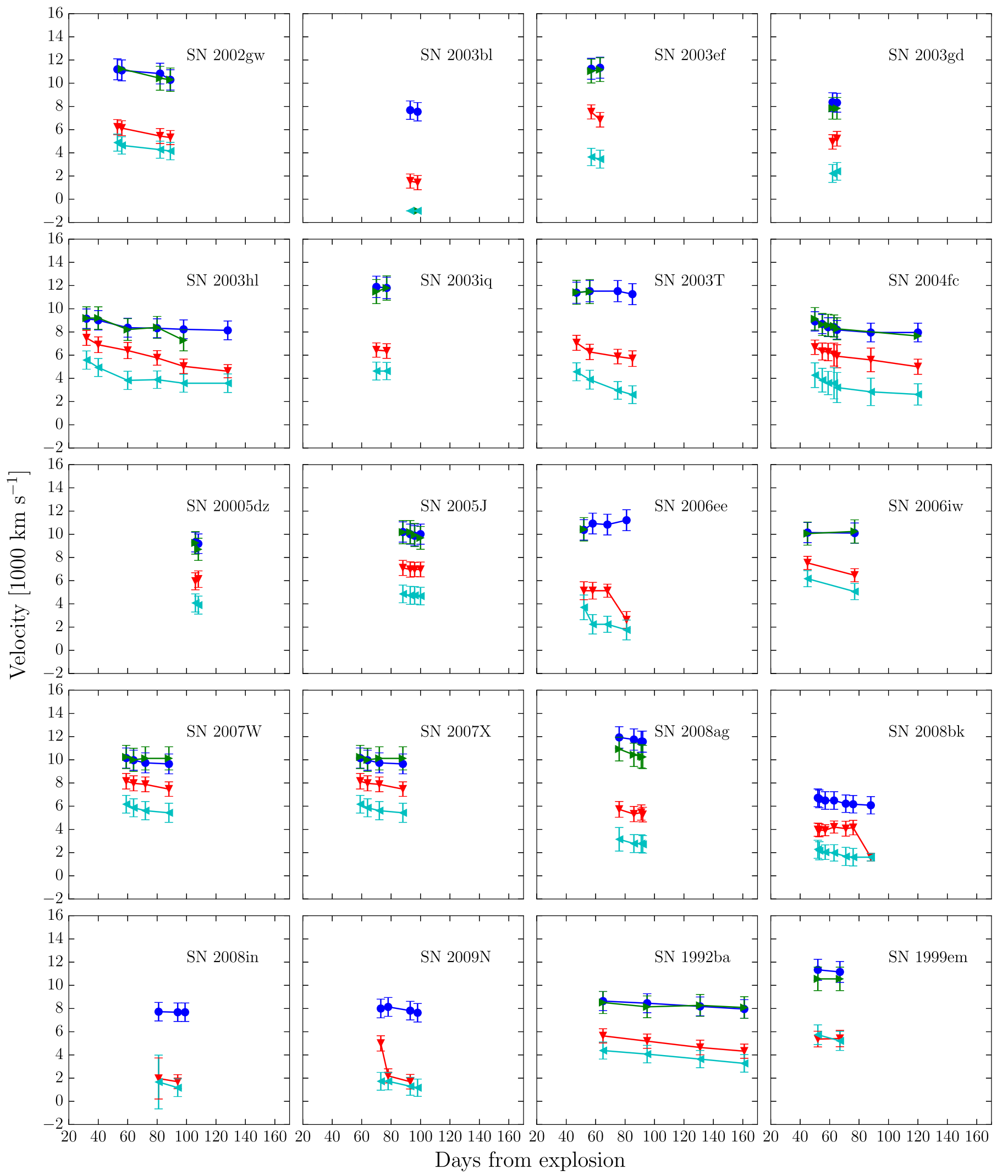

Figure 25. Velocity evolution of Cachito in the plateau phase compared with the Balmer lines. In blue: $\mathrm{HV}$ of $\mathrm{H}_{\alpha}$; in green: $\mathrm{HV}$ of $\mathrm{H}_{\beta}$; in red: the $\mathrm{H}_{\alpha}$ velocity; and in cyan: the $\mathrm{H}_{\beta}$ velocity. 


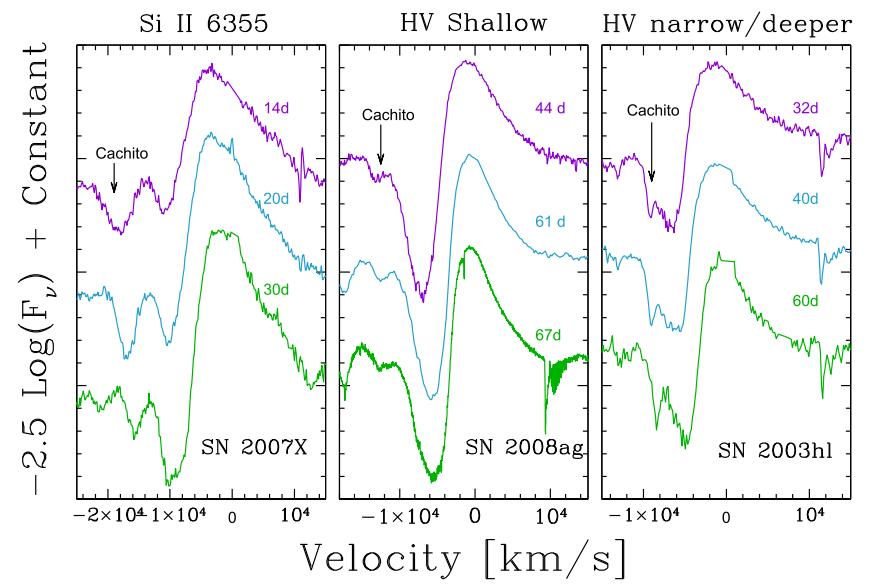

Figure 26. Cachito's shape according to its nature. Left panel: the Si II line in SN 2007X. Middle panel: HV features of $\mathrm{H} \mathrm{I}$ as a shallow absorption in SN 2008ag. Right panel: HV features of H I as narrow and deeper absorption component in SN 2003hl.

$a / e$ values, suggesting that the value of $a / e$ is related to ejecta temperature evolution.

In the case of those $\mathrm{SNe}$ II displaying Cachito consistent with $\mathrm{HV}$ features, these are most likely produced by the interaction of the $\mathrm{SN}$ ejecta with the RSG wind, where the exact shape and persistence of Cachito is related to the wind density Chugai et al. (2007). In Figure 26, one can observe the significant diversity in the different detection of Cachito.

\section{Conclusions}

In this paper, we have presented optical spectra of 122 nearby SNe II observed between 1986 and 2009. A total of 888 spectra ranging between 3 and 363 days post-explosion have been analyzed. The spectral matching technique was discussed as an alternative to nondetection constraints for estimating $\mathrm{SN}$ explosion epochs.

In order to quantify the spectral diversity, we analyze the appearance of the photospheric lines and their time evolution in terms of the $a / e$ and $\mathrm{H}_{\alpha}$ velocity at the $B$-band transition time plus 10 days $\left(t_{\operatorname{tran}+10}\right.$; see Gutiérrez et al. 2014 for more details), the magnitude at maximum $\left(M_{\max }\right)$, the plateau decline $\left(s_{2}\right)$, and metallicity (M13 N2). We analyzed the velocity decline rate of $\mathrm{H}_{\beta}$, the $a / e$ evolution, the expansion ejecta velocities, and the pEWs for 11 features: $\mathrm{H}_{\alpha}, \mathrm{H}_{\beta}, \mathrm{He}$ I/Na I D, Fe II $\lambda 4924$, Fe II $\lambda 5018$, Fe II $\lambda 5169$, Fe II blend, Sc II/Fe II, Sc II multiplet, Ba II, Sc II, and O I. We find a large range in velocities and $\mathrm{pEWs}$, which may be related with a diversity in the explosion energy, radius of the progenitor, and metallicity. The evolution of line strengths was analyzed and compared to that of spectral models. SNe II displaying differences in spectral line evolution were also found to have other different spectral, photometric, and environmental properties. Finally, we discuss the detection and origin of Cachito on the blue side of $\mathrm{H}_{\alpha}$.

The main results obtained with our analysis are summarized as follows.
1. The line evolution indicates differences in temperatures and/or metallicity. Thus, $\mathrm{SNe}$ with slower temperature gradients show the appearance of the iron lines later, while $\mathrm{SNe}$ in environments with higher metallicities show them earlier. In fact, the Fe II line forest is present in faint $\mathrm{SNe}$ with low ejecta temperatures and/or in high metallicity environments. Comparing this result with the synthetic spectra, we find that indeed this feature is only present in higher metallicity (two times solar) and lower explosion energy models, which is consistent with our observations.

2. SNe II display a significant variety of expansion velocities, suggesting a large range in explosion energies.

3. At early phases (before 25 days), $\mathrm{SNe}$ II with a weak $\mathrm{H}_{\alpha}$ absorption component show He I $\lambda 5876$ and the Si II $\lambda 6355$ features. We speculate that this occurs because of higher temperatures at these epochs.

4. Around $60 \%$ of our SNe II show the Cachito feature between 7 and 120 days since explosion. When Cachito is detected less than 30 days post-explosion, then it is identified with Si II. The epochs of early detection can thus inform us of the temperature evolution: SNe II with Si II detections at later epochs have higher temperatures, and this may be related to higher-radius progenitors. At later epochs, during the recombination phase, we suggest that Cachito is related to HV of hydrogen lines. Such HV features are most likely related to the interaction of the SN ejecta with the RSG wind.

All data analyzed in this work are available on http://csp. obs.carnegiescience.edu/, as well as the additional SNID templates (22 $\mathrm{SNe})$, for the $\mathrm{SNe}$ II comparison.

C.P.G. and S.G.G. acknowledge support by projects IC120009 "Millennium Institute of Astrophysics (MAS)" and P10-064-F "Millennium Center for Supernova Science" of the Iniciativa Cientfica Milenio del Ministerio Economa, Fomento y Turismo de Chile. C.P.G. acknowledges support from EU/FP7-ERC grant No. [615929]. M.D.S. is supported by the Danish Agency for Science and Technology and Innovation realized through a Sapere Aude Level 2 grant and by a research grant (13261) from the VILLUM FONDEN. We gratefully acknowledge support of the CSP by the NSF under grants AST0306969, AST0908886, AST0607438, AST1008343, AST-1613472, AST-1613426, and AST-1613455. This research has made use of the NASA/IPAC Extragalactic Database (NED), which is operated by the Jet Propulsion Laboratory, California Institute of Technology, under contract with the National Aeronautics and Space Administration (NASA).

\section{Appendix A Additional Table}

Appendix A presents additional information related to the observations and the analysis of our sample. Table 6 lists the spectroscopic observation information, Table 7 presents the explosion epoch estimation comparison, and Tables 8 and 9 present the mean velocity and mean $\mathrm{pEW}$ values, respectively. 
Table 6

Spectroscopic Observation Information

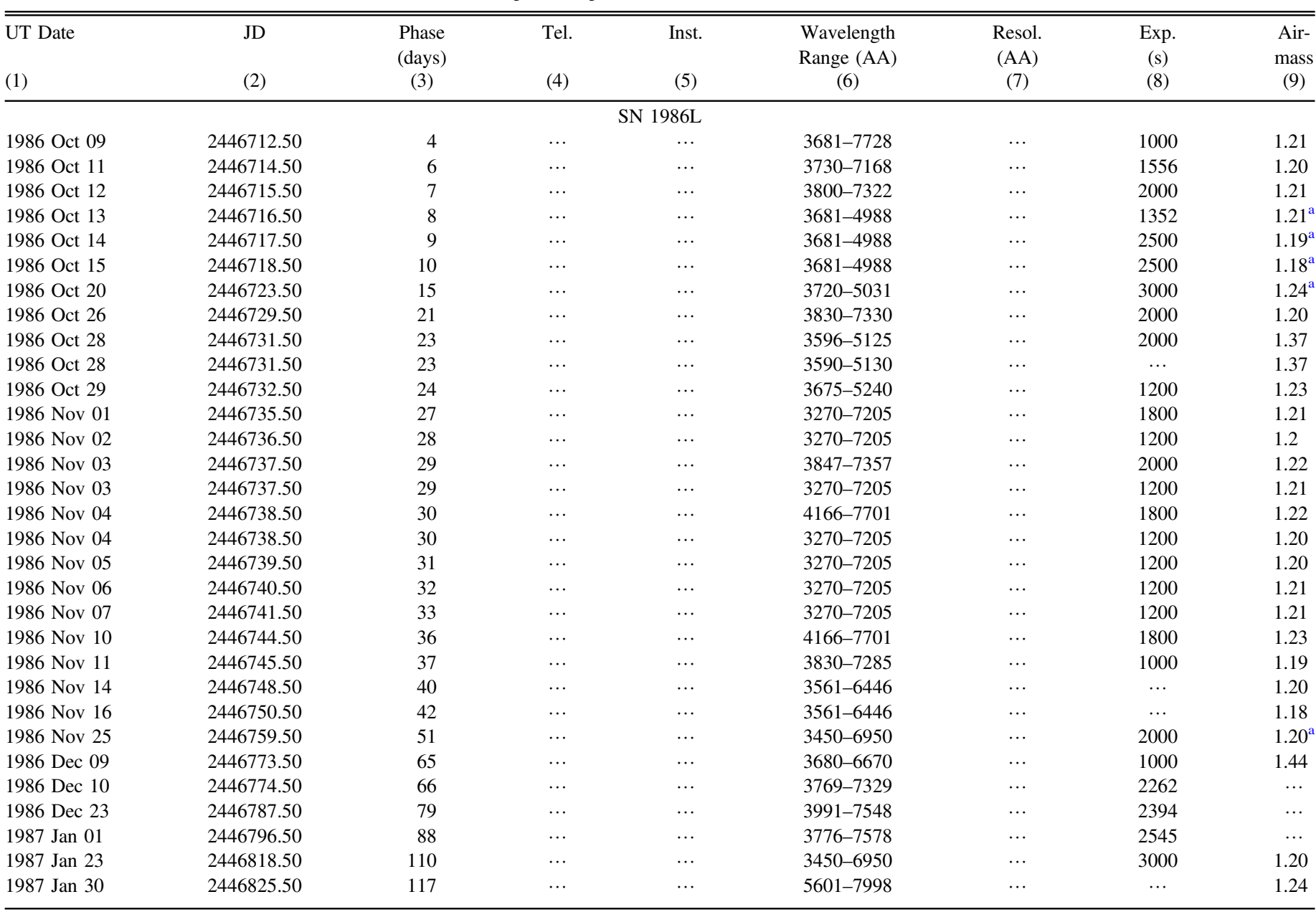

Notes.

Note that up to 1999, we do not have access to the telescope, intrument, and resolution information. Between 2002 and 2003, the resolution information is not available.

Columns: (1) UT date of the observation; (2) Julian date of the observation; (3) phase in days since explosion; (4) telescope code 3P6: ESO 3.6 m Telescope; BAA: Las Campanas Magellan I 6.5 m Baade Telescope; CLA: Las Campanas Magellan II 6.5 m Clay Telescope; DUP: Las Campanas $2.5 \mathrm{~m}$ du Pont Telescope Telescope; NTT: New Technology Telescope; (5) instrument code BC: Boller \& Chivens spectrograph; EF: ESO Faint Object Spectrograph and Camera (EFOSC-2); EM: ESO Multi-Mode Instrument (EMMI); IM: Inamori Magellan Areal Camera and Spectrograph (IMACS), LD: Low Dispersion Survey Spectrograph (LDSS); WF: Wide Field Reimaging CCD Camera (WFCCD); (6) wavelength range covered; (7) spectral resolution in $\AA$ as estimated from arc-lamp lines; (8) total exposure time; (9) airmass at the middle of the observation.

${ }^{\text {a }}$ Spectra with low $\mathrm{S} / \mathrm{N}$.

${ }^{\mathrm{b}}$ Spectra with defects resulting from the observing procedure or data reduction.

(This table is available in its entirety in machine-readable form.) 
Table 7

Explosion Epoch Estimation Comparison

\begin{tabular}{|c|c|c|c|c|c|c|c|c|c|c|}
\hline SN & $\begin{array}{c}\text { Spect. Date } \\
\text { JD }\end{array}$ & Best Match & $\begin{array}{l}\text { Days from } \\
\text { Maximum }\end{array}$ & $\begin{array}{l}\text { Days from } \\
\text { Explosion }\end{array}$ & $\begin{array}{l}\text { Average } \\
\text { (Using } \\
\text { Match) }\end{array}$ & $\begin{array}{c}\text { Explosion Date } \\
\text { (MJD) }\end{array}$ & $\begin{array}{l}\text { Nondetection } \\
\text { Date } \\
\text { (MJD) }\end{array}$ & $\begin{array}{l}\text { Discovery } \\
\text { Date } \\
\text { (MJD) }\end{array}$ & $\begin{array}{c}\text { Explosion Date } \\
\text { (MJD) }\end{array}$ & $\begin{array}{c}\text { Difference } \\
\text { (days) }\end{array}$ \\
\hline $1968 \mathrm{~L}$ & 46715.5 & $\begin{array}{l}2006 \mathrm{bp} \\
1999 \mathrm{em}\end{array}$ & $\begin{array}{l}-2 \\
-4\end{array}$ & $\begin{array}{l}7 \\
6\end{array}$ & 7 & 46708.5 & 46705.5 & 46710.5 & 46708.0 & 0 \\
\hline 1988A & 47188.5 & $\begin{array}{c}1999 \mathrm{em} \\
2006 \mathrm{bp} \\
2004 \mathrm{et}\end{array}$ & $\begin{array}{l}+5 \\
+7 \\
+4\end{array}$ & $\begin{array}{l}15 \\
16 \\
20\end{array}$ & 17 & $47171.5(6)$ & 47175.5 & 47179.0 & $47177.2(2)$ & -6 \\
\hline $1990 \mathrm{E}$ & 47945.5 & $\begin{array}{r}1999 \mathrm{em} \\
2004 \mathrm{et} \\
1999 \mathrm{gi} \\
2006 \mathrm{bp}\end{array}$ & $\begin{array}{r}-3 \\
-3 \\
-4 \\
0\end{array}$ & $\begin{array}{r}7 \\
13 \\
8 \\
9\end{array}$ & 9 & $47936.5(6)$ & 47932.5 & 47937.7 & 47935.1 (3) & 1 \\
\hline $1990 \mathrm{~K}$ & 48049.5 & $\begin{array}{c}\text { 2004et } \\
2006 \mathrm{bp} \\
1999 \mathrm{em}\end{array}$ & $\begin{array}{l}+33 \\
+49 \\
+27\end{array}$ & $\begin{array}{l}49 \\
58 \\
37\end{array}$ & 48 & $48001.5(6)$ & $\ldots$ & 48037.3 & $\ldots$ & $\cdots$ \\
\hline 1991al & 48473.5 & $\begin{array}{c}2006 \mathrm{bp} \\
2004 \mathrm{et} \\
1999 \mathrm{em} \\
2003 \mathrm{iq}\end{array}$ & $\begin{array}{l}+25 \\
+20 \\
+16 \\
\ldots\end{array}$ & $\begin{array}{l}34 \\
36 \\
26 \\
29\end{array}$ & 31 & $48442.5(8)$ & $\cdots$ & 48453.7 & $\cdots$ & $\cdots$ \\
\hline 1992af & 48832.8 & $\begin{array}{r}2003 \mathrm{bn} \\
2007 \mathrm{il} \\
1999 \mathrm{gi} \\
2006 \mathrm{bp} \\
2003 \mathrm{iq} \\
2004 \mathrm{et}\end{array}$ & $\begin{array}{c}\ldots \\
\ldots \\
+19 \\
+20 \\
\ldots \\
+20\end{array}$ & $\begin{array}{l}35 \\
45 \\
31 \\
29 \\
29 \\
36\end{array}$ & 34 & 48798.8 (8) & $\cdots$ & 48802.8 & $\cdots$ & $\cdots$ \\
\hline
\end{tabular}

Note.

Columns: (1) SN name; (2) reduced Julian date of the spectrum used to the match (JD 2,400,000); (3) best match obtained with SNID; (4) days from maximum of the template used to the match; (5) days from explosion of the template used to the match; (6) average obtained from the days from explosion; (7) explosion date obtained with the matching technique; (8) nondetection date of the SN; (9) discovery date of the SN; (10) explosion date obtained from nondetection and discovery date; (11) difference in days between the explosion date from matching technique and nondetection.

(This table is available in its entirety in machine-readable form.) 


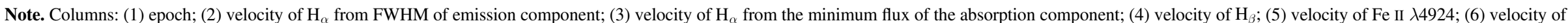
Fe II $\lambda 5018$; (7) velocity of Fe II $\lambda 5169$; (8) velocity of Fe II/Sc II; (9) velocity of Sc II Multiplet; (10) velocity of Na I D; (11) velocity of Ba II; (12) velocity of ScII; and (13) velocity of O I. 
Table 9

Mean pEW Values and the Stardard Deviations for Our Sample

\begin{tabular}{|c|c|c|c|c|c|c|c|c|c|c|c|c|}
\hline $\begin{array}{l}\text { Epoch } \\
\text { (Days) }\end{array}$ & $\begin{array}{l}\mathrm{H}_{\alpha} \\
(\AA)\end{array}$ & $\begin{array}{l}\mathrm{H}_{\alpha} \\
(\AA)\end{array}$ & $\begin{array}{l}\mathrm{H}_{\beta} \\
(\AA)\end{array}$ & $\begin{array}{c}\text { Fe II } \lambda 4924 \\
(\AA)\end{array}$ & $\begin{array}{c}\text { Fe II } \lambda 5018 \\
(\AA)\end{array}$ & $\begin{array}{c}\text { Fe II } \lambda 5169 \\
(\AA)\end{array}$ & $\begin{array}{c}\mathrm{Fe} \text { II/Sc II } \\
(\AA)\end{array}$ & $\begin{array}{l}\text { Sc II Mult. } \\
(\AA)\end{array}$ & $\begin{array}{c}\text { Na I D } \\
(\AA)\end{array}$ & $\begin{array}{c}\text { Ba II } \\
(\AA)\end{array}$ & $\begin{array}{l}\text { Sc II } \\
(\AA)\end{array}$ & $\begin{array}{l}\mathrm{O} \text { I } \\
(\AA)\end{array}$ \\
\hline 4 & $0.8 \pm 1.8$ & $47.3 \pm 28.7$ & $11.9 \pm 8.3$ & $0 \pm 0$ & $0 \pm 0$ & $0 \pm 0$ & $0 \pm 0$ & $0 \pm 0$ & $\cdots$ & $0 \pm 0$ & $0 \pm 0$ & $\cdots$ \\
\hline 8.6 & $4.34 \pm 5.4$ & $83.2 \pm 46.4$ & $24.8 \pm 19.2$ & $0 \pm 0$ & $0 \pm 0$ & $0.1 \pm 0.6$ & $0 \pm 0$ & $0 \pm 0$ & $\cdots$ & $0 \pm 0$ & $0 \pm 0$ & $\cdots$ \\
\hline 12.8 & $8.6 \pm 10.9$ & $121.2 \pm 61.2$ & $33.3 \pm 17.6$ & $0.3 \pm 1.3$ & $1.2 \pm 3.2$ & $5.5 \pm 8.6$ & $0 \pm 0$ & $0 \pm 0$ & $\ldots$ & $0 \pm 0$ & $0 \pm 0$ & $11 \pm 1.1$ \\
\hline 18.1 & $16.6 \pm 17.3$ & $157.2 \pm 53.7$ & $37.4 \pm 14.8$ & $0.2 \pm 0.8$ & $4.2 \pm 4.8$ & $14.5 \pm 14.0$ & $0 \pm 0$ & $0 \pm 0$ & $\cdots$ & $0 \pm 0$ & $0 \pm 0$ & $5.67 \pm 1.1$ \\
\hline 23.1 & $25.7 \pm 22.1$ & $147.8 \pm 62.2$ & $43.3 \pm 18.8$ & $1.4 \pm 2.5$ & $9.3 \pm 5.5$ & $22.4 \pm 10.9$ & $1.1 \pm 2.5$ & $1.02 \pm 3.21$ & $\cdots$ & $0.1 \pm 0.3$ & $0.1 \pm 0.30$ & $11.5 \pm 8.6$ \\
\hline 27.7 & $25.0 \pm 25.9$ & $142.7 \pm 55.2$ & $43.8 \pm 16.7$ & $1.1 \pm 2.5$ & $9.6 \pm 4.5$ & $25.5 \pm 9.5$ & $1.4 \pm 3.1$ & $1.61 \pm 3.99$ & $\cdots$ & $0.3 \pm 1.0$ & $0.5 \pm 1.40$ & $10.9 \pm 5.6$ \\
\hline 33.1 & $36.6 \pm 20.4$ & $155.7 \pm 44.5$ & $49.2 \pm 13.6$ & $2.8 \pm 3.6$ & $12.5 \pm 4.0$ & $30.2 \pm 8.4$ & $4.9 \pm 4.4$ & $6.48 \pm 6.30$ & $13.3 \pm 7.6$ & $1.1 \pm 2.7$ & $2.1 \pm 3.17$ & $9.8 \pm 5.8$ \\
\hline 38.1 & $42.5 \pm 23.3$ & $152.9 \pm 44.2$ & $50.2 \pm 16.4$ & $3.8 \pm 3.8$ & $14.5 \pm 6.0$ & $33 \pm 13.5$ & $6.0 \pm 5.0$ & $8.09 \pm 7.14$ & $15.6 \pm 8.3$ & $2.0 \pm 3.4$ & $2.8 \pm 4.00$ & $10.6 \pm 3.9$ \\
\hline 42.8 & $46.1 \pm 22.7$ & $142.0 \pm 64.0$ & $49.2 \pm 15.9$ & $6.1 \pm 4.2$ & $15.1 \pm 5.4$ & $32.9 \pm 9.2$ & $7.1 \pm 4.8$ & $10.5 \pm 5.87$ & $18.7 \pm 10.3$ & $2.8 \pm 3.9$ & $3.7 \pm 3.77$ & $11.0 \pm 5.5$ \\
\hline 47.8 & $48.1 \pm 21.6$ & $169 \pm 61.7$ & $54.2 \pm 18.3$ & $6.4 \pm 4.1$ & $14.2 \pm 5.8$ & $34.6 \pm 9.6$ & $7.9 \pm 3.3$ & $11.5 \pm 4.83$ & $26.5 \pm 12.5$ & $4.3 \pm 4.7$ & $5.1 \pm 3.26$ & $13.0 \pm 4.9$ \\
\hline 53.1 & $56.6 \pm 21.7$ & $156.9 \pm 55.2$ & $48.8 \pm 20.3$ & $8.1 \pm 5.5$ & $17.9 \pm 5.9$ & $40.2 \pm 13.5$ & $10.1 \pm 4.7$ & $14.0 \pm 7.53$ & $32.4 \pm 15.2$ & $5.6 \pm 4.7$ & $7.2 \pm 5.64$ & $11.9 \pm 5.9$ \\
\hline 58.5 & $50.7 \pm 24.9$ & $169.7 \pm 78.6$ & $49.6 \pm 26.4$ & $7.5 \pm 4.7$ & $18.3 \pm 7.4$ & $39.8 \pm 15.7$ & $11.2 \pm 5.5$ & $16.0 \pm 8.35$ & $38.3 \pm 20.0$ & $6.4 \pm 6.2$ & $6.7 \pm 5.09$ & $11.1 \pm 5.8$ \\
\hline 63.3 & $58.1 \pm 18.4$ & $173.3 \pm 70.1$ & $49.5 \pm 25.1$ & $9.7 \pm 5.7$ & $19.8 \pm 5.6$ & $44.1 \pm 11.0$ & $11.8 \pm 5.3$ & $18.2 \pm 7.63$ & $46.0 \pm 17.5$ & $7.9 \pm 6.8$ & $7.4 \pm 4.78$ & $10.8 \pm 4.6$ \\
\hline 68.0 & $60.2 \pm 17.3$ & $163.3 \pm 42.1$ & $53.1 \pm 27.0$ & $9.4 \pm 6.0$ & $21.6 \pm 7.0$ & $42.8 \pm 9.6$ & $13.6 \pm 6.1$ & $19.5 \pm 7.79$ & $52.2 \pm 17.1$ & $8.2 \pm 6.3$ & $8.0 \pm 5.88$ & $12.1 \pm 4.5$ \\
\hline 72.8 & $65.2 \pm 20.8$ & $179.5 \pm 71.8$ & $56.3 \pm 32.2$ & $9.6 \pm 6.3$ & $19.5 \pm 6.3$ & $41 \pm 11.7$ & $11.5 \pm 5.9$ & $17.3 \pm 8.15$ & $49.4 \pm 24.5$ & $8.1 \pm 6.9$ & $6.9 \pm 5.61$ & $14.3 \pm 7.1$ \\
\hline 78.2 & $60.0 \pm 21.4$ & $167.0 \pm 71.7$ & $46.9 \pm 26.1$ & $11.6 \pm 6.6$ & $20.8 \pm 7.8$ & $42.4 \pm 10.1$ & $14.7 \pm 5.2$ & $22.9 \pm 7.55$ & $59.7 \pm 21.8$ & $12.6 \pm 7.56$ & $11.4 \pm 4.7$ & $12.7 \pm 5.6$ \\
\hline 83.5 & $53.8 \pm 31.1$ & $202.2 \pm 86.1$ & $52.4 \pm 27.9$ & $10.4 \pm 5.9$ & $21.5 \pm 7.8$ & $47.0 \pm 13.5$ & $14.3 \pm 5.2$ & $21.3 \pm 8.00$ & $63.9 \pm 28.6$ & $12.8 \pm 9.41$ & $11.4 \pm 7.4$ & $11.1 \pm 3.7$ \\
\hline 87.5 & $56.1 \pm 26.9$ & $176.4 \pm 95.0$ & $55.2 \pm 28.8$ & $10.5 \pm 7.6$ & $23.0 \pm 7.5$ & $51.3 \pm 12.8$ & $14.9 \pm 6.2$ & $21.6 \pm 9.26$ & $63.2 \pm 19.0$ & $11.4 \pm 7.67$ & $10.1 \pm 6.5$ & $15.4 \pm 6.9$ \\
\hline 93.3 & $50.9 \pm 28.8$ & $182.9 \pm 107.3$ & $47.0 \pm 26.7$ & $13.3 \pm 8.1$ & $25.6 \pm 8.2$ & $48.2 \pm 11.1$ & $17.3 \pm 7.7$ & $27.3 \pm 10.6$ & $69.7 \pm 17.7$ & $16.6 \pm 12.4$ & $12.4 \pm 6.5$ & $11.5 \pm 5.8$ \\
\hline 98.2 & $61.6 \pm 28.3$ & $214.4 \pm 117.6$ & $62.7 \pm 29.3$ & $14.9 \pm 4.7$ & $26.2 \pm 7.0$ & $46.4 \pm 9.9$ & $17.7 \pm 6.2$ & $27.6 \pm 7.81$ & $81.8 \pm 26.4$ & $17.6 \pm 10.2$ & $12.9 \pm 6.7$ & $12.4 \pm 2.7$ \\
\hline 103.0 & $48.2 \pm 25.9$ & $184.8 \pm 80.3$ & $41.2 \pm 24.8$ & $19.1 \pm 4.7$ & $30.7 \pm 3.9$ & $48.8 \pm 6.4$ & $16.8 \pm 6.6$ & $26.4 \pm 9.00$ & $76.0 \pm 14.9$ & $34.6 \pm 9.78$ & $16.5 \pm 4.0$ & $11.8 \pm 1.6$ \\
\hline 108.2 & $60.1 \pm 19.0$ & $208.9 \pm 56.1$ & $43.8 \pm 15.5$ & $15.9 \pm 4.0$ & $25.7 \pm 2.6$ & $41 \pm 10.2$ & $15.0 \pm 0.0$ & $21.5 \pm 4.11$ & $69.2 \pm 20.8$ & $20.3 \pm 15.7$ & $9.8 \pm 2.7$ & $13.0 \pm 2.8$ \\
\hline 115.7 & $46.2 \pm 18.6$ & $287.5 \pm 158.8$ & $53.4 \pm 29.4$ & $7.9 \pm 0.9$ & $16.8 \pm 6.2$ & $35.1 \pm 12.9$ & $21.2 \pm 3.5$ & $16.3 \pm 4.5$ & $70.2 \pm 23.1$ & $22.4 \pm 15.7$ & $6.7 \pm 4.5$ & $8.9 \pm 1.5$ \\
\hline
\end{tabular}

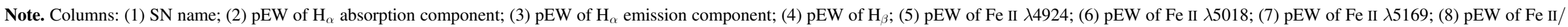
Sc II; (9) pEW of Sc II Multiplet; (10) pEW of Na I D; (11) pEW of Ba II; (12) pEW of ScII; (13) pEW of O I. 


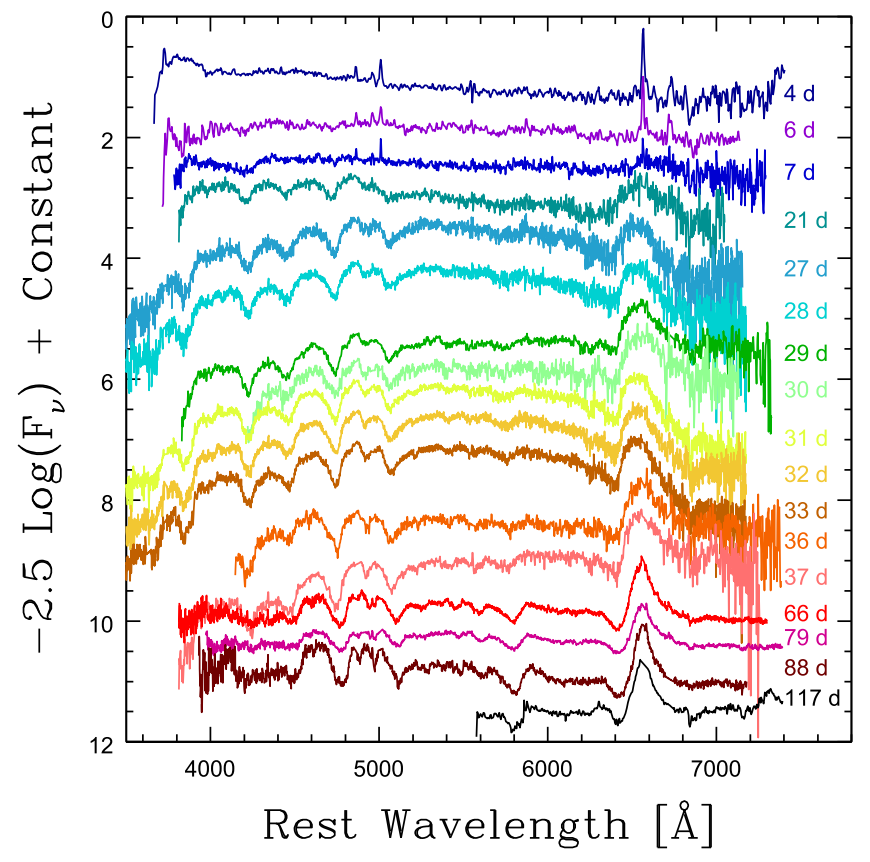

Figure 27. SNe II SN 1986L spectra.

(The complete figure set (120 images) is available.)
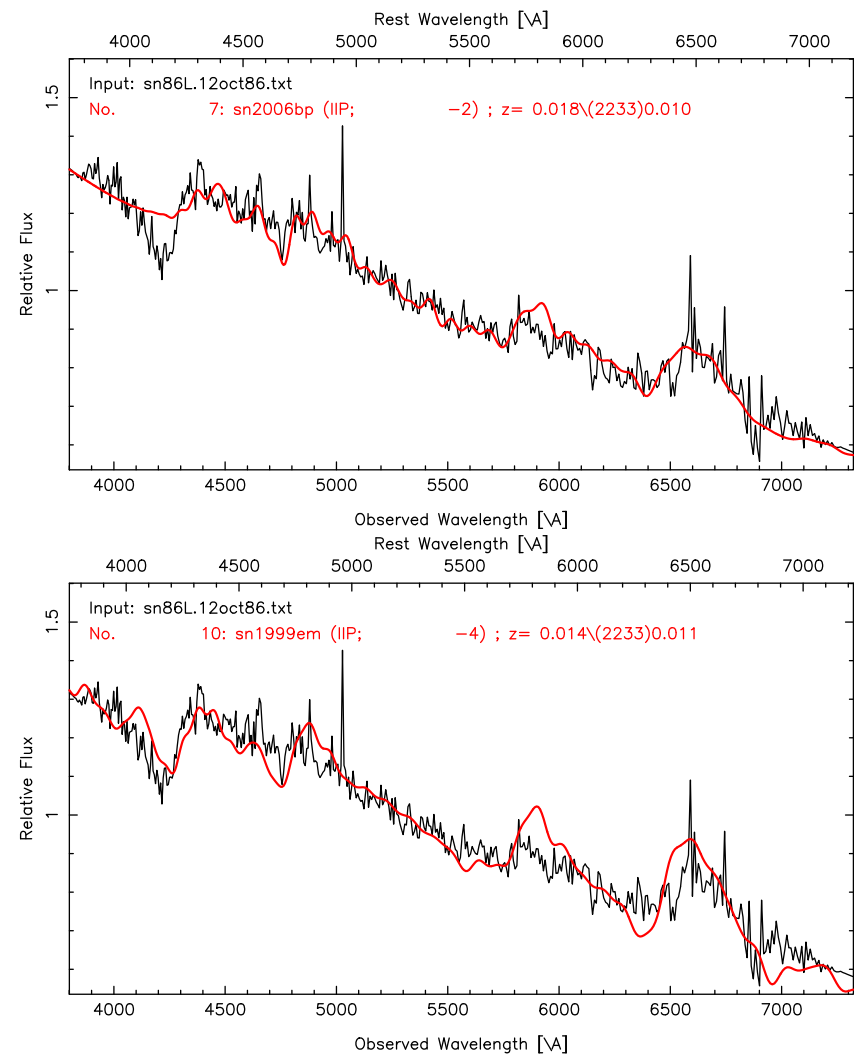

Figure 28. Best spectral matching of SN 1986L using SNID. The plots show SN 1986L compared with SN 2006bp and SN 1999em at 6 and 7 days from explosion.

(The complete figure set (430 images) is available.)

\section{Appendix B Spectral Series}

In this section, the spectral time-series for all $\mathrm{SNe}$ in our sample are presented. Figure 27 shows an example of the spectral evolution of SN 1986L. Plots for the full sample can be found in the online version.

\section{Appendix C SNID Matches}

In this section, we present the best spectral matching plots for each SN in our sample. An example of this technique is shown in Figure 28 for SN 1986L. Plots for the full sample can be found in the online version.

\section{ORCID iDs}

Claudia P. Gutiérrez (10) https://orcid.org/0000-00027252-4351

Joseph P. Anderson (10 https://orcid.org/0000-0003-0227-3451 Nidia Morrell (1) https://orcid.org/0000-0003-2535-3091 Maximilian D. Stritzinger (ib https://orcid.org/0000-00025571-1833

Mark M. Phillips (ㄷ) https://orcid.org/0000-0003-2734-0796

Lluis Galbany (1) https://orcid.org/0000-0002-1296-6887

Luc Dessart (10) https://orcid.org/0000-0003-0599-8407 Massimo Della Valle iㅏ https://orcid.org/0000-00033142-5020

Eric Y. Hsiao (1) https://orcid.org/0000-0003-1039-2928

Kevin Krisciunas (1) https://orcid.org/0000-0002-6650-694X Barry F. Madore (1) https://orcid.org/0000-0002-1576-1676 Jose Luis Prieto (1) https://orcid.org/0000-0003-0943-0026 Enrico Cappellaro (i) https://orcid.org/0000-0001-5008-8619 Alessandro Pizzella (1) https://orcid.org/0000-00019585-417X

\section{References}

Allington-Smith, J., Breare, M., Ellis, R., et al. 1994, PASP, 106, 983 Anderson, J. P., Dessart, L., Gutierrez, C. P., et al. 2014a, MNRAS, 441, 671 Anderson, J. P., Gutiérrez, C. P., Dessart, L., et al. 2016, A\&A, 589, A110 Anderson, J. P., González-Gaitán, S., Hamuy, M., et al. 2014b, ApJ, 786, 67 Arcavi, I., Gal-Yam, A., Kasliwal, M. M., et al. 2010, ApJ, 721, 777 Barbon, R., Buondí, V., Cappellaro, E., \& Turatto, M. 1999, A\&AS, 139, 531 Barbon, R., Ciatti, F., \& Rosino, L. 1979, A\&A, 72, 287

Baron, E., Branch, D., Hauschildt, P. H., et al. 2000, ApJ, 545, 444 Blanco, V. M., Gregory, B., Hamuy, M., et al. 1987, ApJ, 320, 589 Blondin, S., \& Tonry, J. L. 2007, ApJ, 666, 1024

Bose, S., Kumar, B., Sutaria, F., et al. 2013, MNRAS, 433, 1871

Branch, D., Falk, S. W., Uomoto, A. K., et al. 1981, ApJ, 244, 780 Buta, R. J. 1982, PASP, 94, 578

Buzzoni, B., Delabre, B., Dekker, H., et al. 1984, Msngr, 38, 9 Cappellaro, E., Danziger, I. J., della Valle, M., Gouiffes, C., \& Turatto, M. 1995, A\&A, 293, 723

Chugai, N. N., Chevalier, R. A., \& Utrobin, V. P. 2007, ApJ, 662, 1136 Contreras, C., Hamuy, M., Phillips, M. M., et al. 2010, AJ, 139, 519 Dall'Ora, M., Botticella, M. T., Pumo, M. L., et al. 2014, ApJ, 787, 139 Dekker, H., Delabre, B., \& Dodorico, S. 1986, Proc. SPIE, 627, 339 Dessart, L., Blondin, S., Brown, P. J., et al. 2008, ApJ, 675, 644 Dessart, L., \& Hillier, D. J. 2005, A\&A, 437, 667

Dessart, L., \& Hillier, D. J. 2006, A\&A, 447, 691

Dessart, L., \& Hillier, D. J. 2008, MNRAS, 383, 57

Dessart, L., \& Hillier, D. J. 2010, MNRAS, 405, 2141

Dessart, L., \& Hillier, D. J. 2011, MNRAS, 410, 1739

Dessart, L., Hillier, D. J., Waldman, R., \& Livne, E. 2013, MNRAS, 433, 1745

Dressler, A., Bigelow, B., Hare, T., et al. 2011, PASP, 123, 288 
Dwek, E. 1983, ApJ, 274, 175

Fabbri, J., Otsuka, M., Barlow, M. J., et al. 2011, MNRAS, 418, 1285 Faran, T., Poznanski, D., Filippenko, A. V., et al. 2014a, MNRAS, 445, 554 Faran, T., Poznanski, D., Filippenko, A. V., et al. 2014b, MNRAS, 442, 844 Fesen, R. A., Gerardy, C. L., Filippenko, A. V., et al. 1999, AJ, 117, 725 Filippenko, A. V., Matheson, T., \& Ho, L. C. 1993, ApJL, 415, L103 Folatelli, G., Morrell, N., Phillips, M. M., et al. 2013, ApJ, 773, 53 Folatelli, G., Phillips, M. M., Burns, C. R., et al. 2010, AJ, 139, 120 Fransson, C., \& Chevalier, R. A. 1987, ApJL, 322, L15

Galbany, L., Hamuy, M., Phillips, M. M., et al. 2016, AJ, 151, 33 Gutiérrez, C. P., Anderson, J. P., Hamuy, M., et al. 2014, ApJL, 786, L15 Hamuy, M. 2003, ApJ, 582, 905

Hamuy, M., Folatelli, G., Morrell, N. I., et al. 2006, PASP, 118, 2

Hamuy, M., Maza, J., Phillips, M. M., et al. 1993, AJ, 106, 2392

Hamuy, M., Phillips, M. M., Suntzeff, N. B., et al. 1996, AJ, 112, 2438

Hamuy, M., \& Pinto, P. A. 2002, ApJL, 566, L63

Hamuy, M., Pinto, P. A., Maza, J., et al. 2001, ApJ, 558, 615

Hamuy, M., Suntzeff, N. B., Gonzalez, R., \& Martin, G. 1988, AJ, 95, 63

Hamuy, M. A. 2001, PhD thesis, Univ. Arizona

Harutyunyan, A. H., Pfahler, P., Pastorello, A., et al. 2008, A\&A, 488, 383

Howell, D. A., Sullivan, M., Perrett, K., et al. 2005, ApJ, 634, 1190

Immler, S., Fesen, R. A., Van Dyk, S. D., et al. 2005, ApJ, 632, 283

Inserra, C., Pastorello, A., Turatto, M., et al. 2013, A\&A, 555, A142

Inserra, C., Turatto, M., Pastorello, A., et al. 2011, MNRAS, 417, 261

Inserra, C., Turatto, M., Pastorello, A., et al. 2012, MNRAS, 422, 1122

Jerkstrand, A., Fransson, C., Maguire, K., et al. 2012, A\&A, 546, A28

Jerkstrand, A., Smartt, S. J., Fraser, M., et al. 2014, MNRAS, 439, 3694

Jones, M. I., Hamuy, M., Lira, P., et al. 2009, ApJ, 696, 1176

Kotak, R., Meikle, W. P. S., Farrah, D., et al. 2009, ApJ, 704, 306

Leonard, D. C., Filippenko, A. V., Gates, E. L., et al. 2002b, PASP, 114, 35

Leonard, D. C., Filippenko, A. V., Li, W., et al. 2002a, AJ, 124, 2490

Li, W., Van Dyk, S. D., Filippenko, A. V., \& Cuillandre, J.-C. 2005, PASP, 117,121

Lisakov, S. M., Dessart, L., Hillier, D. J., Waldman, R., \& Livne, E. 2017, MNRAS, 466, 34

Maguire, K., Di Carlo, E., Smartt, S. J., et al. 2010, MNRAS, 404, 981

Marino, R. A., Rosales-Ortega, F. F., Sánchez, S. F., et al. 2013, A\&A, 559, A114

Maund, J. R., \& Smartt, S. J. 2005, MNRAS, 360, 288

Menzies, J. W., Catchpole, R. M., van Vuuren, G., et al. 1987, MNRAS, 227, 39P

Minkowski, R. 1941, PASP, 53, 224
Misra, K., Pooley, D., Chandra, P., et al. 2007, MNRAS, 381, 280

Müller, T., Prieto, J. L., Pejcha, O., \& Clocchiatti, A. 2017, ApJ, 841, 127

Olivares, F. 2008, MSc thesis, Univ. Chile (arXiv:0810.5518)

Pastorello, A., Sauer, D., Taubenberger, S., et al. 2006, MNRAS, 370 1752

Pastorello, A., Valenti, S., Zampieri, L., et al. 2009, MNRAS, 394, 2266

Pastorello, A., Zampieri, L., Turatto, M., et al. 2004, MNRAS, 347, 74

Patat, F., Barbon, R., Cappellaro, E., \& Turatto, M. 1994, A\&A, 282, 731

Pejcha, O., \& Prieto, J. L. 2015a, ApJ, 799, 215

Pejcha, O., \& Prieto, J. L. 2015b, ApJ, 806, 225

Phillips, M. M., Heathcote, S. R., Hamuy, M., \& Navarrete, M. 1988, AJ, 95, 1087

Pooley, D., Lewin, W. H. G., Fox, D. W., et al. 2002, ApJ, 572, 932

Roy, R., Kumar, B., Benetti, S., et al. 2011, ApJ, 736, 76

Sahu, D. K., Anupama, G. C., Srividya, S., \& Muneer, S. 2006, MNRAS, 372,1315

Sanders, N. E., Soderberg, A. M., Gezari, S., et al. 2015, ApJ, 799, 208

Schlafly, E. F., \& Finkbeiner, D. P. 2011, ApJ, 737, 103

Schlegel, E. M. 1990, MNRAS, 244, 269

Schmidt, B. P., Kirshner, R. P., Schild, R., et al. 1993, AJ, 105, 2236

Smartt, S. J. 2015, PASA, 32, e016

Smartt, S. J., Eldridge, J. J., Crockett, R. M., \& Maund, J. R. 2009, MNRAS, 395, 1409

Smartt, S. J., Maund, J. R., Hendry, M. A., et al. 2004, Sci, 303, 499

Spiro, S., Pastorello, A., Pumo, M. L., et al. 2014, MNRAS, 439, 2873

Stritzinger, M. D., Anderson, J. P., Contreras, C., et al. 2017, arXiv:1707.07616

Stritzinger, M. D., Phillips, M. M., Boldt, L. N., et al. 2011, AJ, 142, 156

Suntzeff, N. B., Hamuy, M., Martin, G., Gomez, A., \& Gonzalez, R. 1988, AJ, 96, 1864

Taddia, F., Stritzinger, M. D., Bersten, M., et al. 2017, A\&A, submitted (arXiv:1707.07614)

Taddia, F., Stritzinger, M. D., Sollerman, J., et al. 2012, A\&A, 537 A140

Taddia, F., Stritzinger, M. D., Sollerman, J., et al. 2013, A\&A, 555, A10

Tomasella, L., Cappellaro, E., Fraser, M., et al. 2013, MNRAS, 434, 1636

Turatto, M., Cappellaro, E., Benetti, S., \& Danziger, I. J. 1993, MNRAS, 265,471

Valenti, S., Howell, D. A., Stritzinger, M. D., et al. 2016, MNRAS, 459 3939

Valenti, S., Sand, D., Pastorello, A., et al. 2014, MNRAS, 438, L101

Van Dyk, S. D., Li, W., \& Filippenko, A. V. 2003, PASP, 115, 1289

Wood-Vasey, W. M., Aldering, G., Lee, B. C., et al. 2004, NewAR, 48, 637 